\title{
Rechtliche Grundlagen im medizinischen Alltag
}

Ein Leitfaden für die Praxis 


\section{Herausgeber}

Schweizerische Akademie der Medizinischen Wissenschaften

Petersplatz 13, $\mathrm{CH}-4051$ Basel

T +416126990 30, mail@samw.ch, www.samw.ch

Verbindung der Schweizer Ärztinnen und Ärzte FMH

Elfenstr. 18, CH-3000 Bern 15

T +41313591111, lex@fmh.ch, www.fmh.ch

\section{Grafik}

Howald Fosco, Basel

\section{Fotos}

Howald Fosco, Basel

ausser S. 135: @ Dmitry Pistrov - Fotolia.com

\section{Druck}

Schwabe Druckerei, Muttenz

2. überarbeitete Auflage, 2013

Die Broschüre kann kostenlos bezogen werden bei der SAMW.

(c) SAMW/FMH 2013 


\section{Rechtliche Grundlagen im medizinischen Alltag}

Ein Leitfaden für die Praxis

2., überarbeitete und um den Bereich "Erwachsenenschutzrecht» erweiterte Auflage. Herausgegeben von der Schweizerischen Akademie der Medizinischen Wissenschaften und der Verbindung der Schweizer Ärztinnen und Ärzte FMH. 

1. GRUNDLAGEN DES RECHTS 9

1.1. Die Funktion des Rechts im ärztlichen Alltag 9

1.2. Stufen des Rechts 10

1.3. Die privaten Regelwerke 12

1.4. Neue Entwicklungen 13

2. RAHMENBEDINGUNGEN DER ÄRZTLICHEN TÄTIGKEIT 16

2.1. Aus-, Weiter- und Fortbildung 16

2.2. Das Medizinalberufegister 19

2.3. Die Berufs- und Kassenzulassung 20

2.4. Berufspflichten 20

2.5. Werbung und Ausschreibung 23

2.6. Sozial- und Privatversicherungen 24

3. ERWACHSENENSCHUTZRECHT 27

3.1. Eigene Vorsorge 27

3.2. Massnahmen von Gesetzes wegen 28

3.3. Aufenthalt in Wohn- und Pflegeeinrichtungen 29

3.4. Behördliche Massnahmen 29

3.5. Die Behörden des Erwachsenenschutzes 31

3.6. Verfahren 32

4. GRUNDLAGEN DER BEHANDLUNG VON PATIENTINNEN $\begin{array}{ll}\text { UND PATIENTEN } & 34\end{array}$

4.1. Behandlungsvertrag zwischen Arzt und Patient 34

4.2. Aufklärung des Patienten $\quad 40$

4.3. Einwilligung 43

4.4. Patientenverfügung 45

4.5. Führung der Krankengeschichte 46

4.6. Arzneimittel, Betäubungsmittel und Medizinprodukte 48

4.7. "Off label use» und "unlicensed use» 51

4.8. Nichtetablierte Behandlungen bei einzelnen Patienten 52

4.9. Röntgenuntersuchungen und Labor 52

4.10. Grundprinzipien der obligatorischen Krankenpflegeversicherung 54

4.11. Leistungserbringer und Wahlfreiheit im KVG 56 
4.12. Managed Care 58

4.13. Qualitätssicherung und Register 59

4.14. Kostenübernahme durch die Krankenpflegeversicherung von
Arzneimitteln, Mitteln und Gegenständen im KVG

4.15. Tarifrecht und Verfahren bei Streitigkeiten im KVG 65

4.16. Behandlung von Patienten in der Unfall-, Militär- und
Invalidenversicherung

5. SPEZIELLE SITUATIONEN BEI DER BEHANDLUNG VON PATIENTINNEN UND PATIENTEN 74

5.1. Genetische Untersuchungen 74

5.2. Fortpflanzungsmedizin 76

5.3. Schwangerschaftsabbruch $\quad 77$

5.4. Sterilisation $\quad 77$

5.5. Transplantationsmedizin 78

5.6. Ärztliche Betreuung von Sportlern; Doping 79

5.7. Behandlung und Betreuung von älteren, pflegebedürftigen Menschen 80

5.8. Medizinische Entscheidungen am Lebensende 81

5.9. Beihilfe zum Suizid 84

5.10.Zwangsmassnahmen in der Medizin 86

5.11. Behandlung von Patienten im Strafvollzug 89

5.12. Behandlung von Sans-Papiers und Asylsuchenden 91

6. FORSCHUNG 93

6.1. Forschung am Menschen 93

6.2. Stammzellenforschung 94

6.3. Wissenschaftliche Integrität 95

7. DER ARZT ALS BERICHTERSTATTER: VOM ARZTGEHEIMNIS ZUR RECHNUNGSSTELLUNG 98

7.1. Arztgeheimnis (Berufsgeheimnis, Patientengeheimnis) 98

$\begin{array}{ll}\text { 7.2. Datenschutz } & 102\end{array}$

7.3. Meldepflichten und Melderechte 104

7.4. Allgemeines zu Arztzeugnis und Bericht 108

7.5. Berichte an mitbehandelnde Ärzte 108

7.6. Zeugnisse und Berichte an den Arbeitgeber und den Taggeldversicherer 109

7.7. Berichte und Abrechnung in der Krankenversicherung 111 
7.8. Berichte und Abrechnung im UVG, MVG und IVG

7.9. Zeugnisse und Berichte an Pensionskassen und Lebensversicherungen 119 7.10 .

8. DER ARZT ALS GUTACHTER

8.1. Allgemeines zum ärztlichen Gutachten

8.2. Ärztliche Gutachten für die Sozialversicherung

9. RECHENSCHAFTSPFLICHT UND VERANTWORTLICHKEIT DES ARZTES

9.1. Rechenschaftspflicht

9.2. Einsichts- und Kopierrechte des Patienten an der Krankengeschichte 128

9.3. Die Verantwortlichkeit des Arztes - Haftung im Zivil- und Strafrecht 130

9.4. Der unzufriedene Patient

10. DER ARZT ALS ARBEITNEHMER ODER ARBEITGEBER

10.1. Anstellung am Spital oder in der Praxis

10.2. Der selbstständig tätige Arzt: Praxiseröffnung, Praxisübernahme und Praxisschliessung

10.3. Praxisführung

10.4. Pflichten als Arbeitgeber

10.5. Jugendarbeitsschutz und Mutterschutz 


\section{Vorwort}

\section{zur 2., überarbeiteten Auflage}

Der ärztliche Alltag ist von zahlreichen rechtlichen Bestimmungen begleitet. Oft ist es für eine Ärztin oder einen Arzt schwierig, einen Überblick über die relevanten Gesetzestexte zu bewahren; die allgemeinen rechtlichen Regeln erweisen sich zudem häufig als zu vage, als dass daraus konkrete Verhaltensleitlinien abgeleitet werden könnten.

Mit der Broschüre «Rechtliche Grundlagen im medizinischen Alltag» möchten die SAMW und die FMH hier eine Hilfestellung bieten. Das neue Erwachsenenschutzrecht, das das frühere Vormundschaftsrecht abgelöst hat, war der Anlass, den Leitfaden in zweiter Auflage herauszugeben. Den Leserinnen und Lesern soll dieser Leitfaden eine Übersicht bieten über jene rechtlichen Regelungen, welche für den medizinischen Alltag relevant sind. Sein Nutzen für den Praxisalltag besteht darin, dass er häufige und geläufige Fragen einfach und anwendungsorientiert beantwortet. Gesetzestexte und Urteile sind bis Ende 2012 nachgeführt.

Die Texte sind bewusst kurz gehalten und sollen einen ersten Einstieg in die Thematik erlauben. Der Leitfaden ist auf der Website der FMH* abrufbar. Er kann dort nach Stichworten durchsucht werden und wird mit weiterführenden Hinweisen und aktuellen Informationen ergänzt.

Gerne danken wir an dieser Stelle den zahlreichen Autorinnen und Autoren für ihre Beiträge sowie der wissenschaftlichen Begleitgruppe für die sorgfältige Durchsicht des Manuskriptes; ohne sie wäre die Realisierung dieser Broschüre nicht möglich gewesen.

Dr. Jürg Schlup

Präsident FMH
Prof. Peter Meier-Abt

Präsident SAMW

* www.fmh.ch $\rightarrow$ Service $\rightarrow$ Recht 


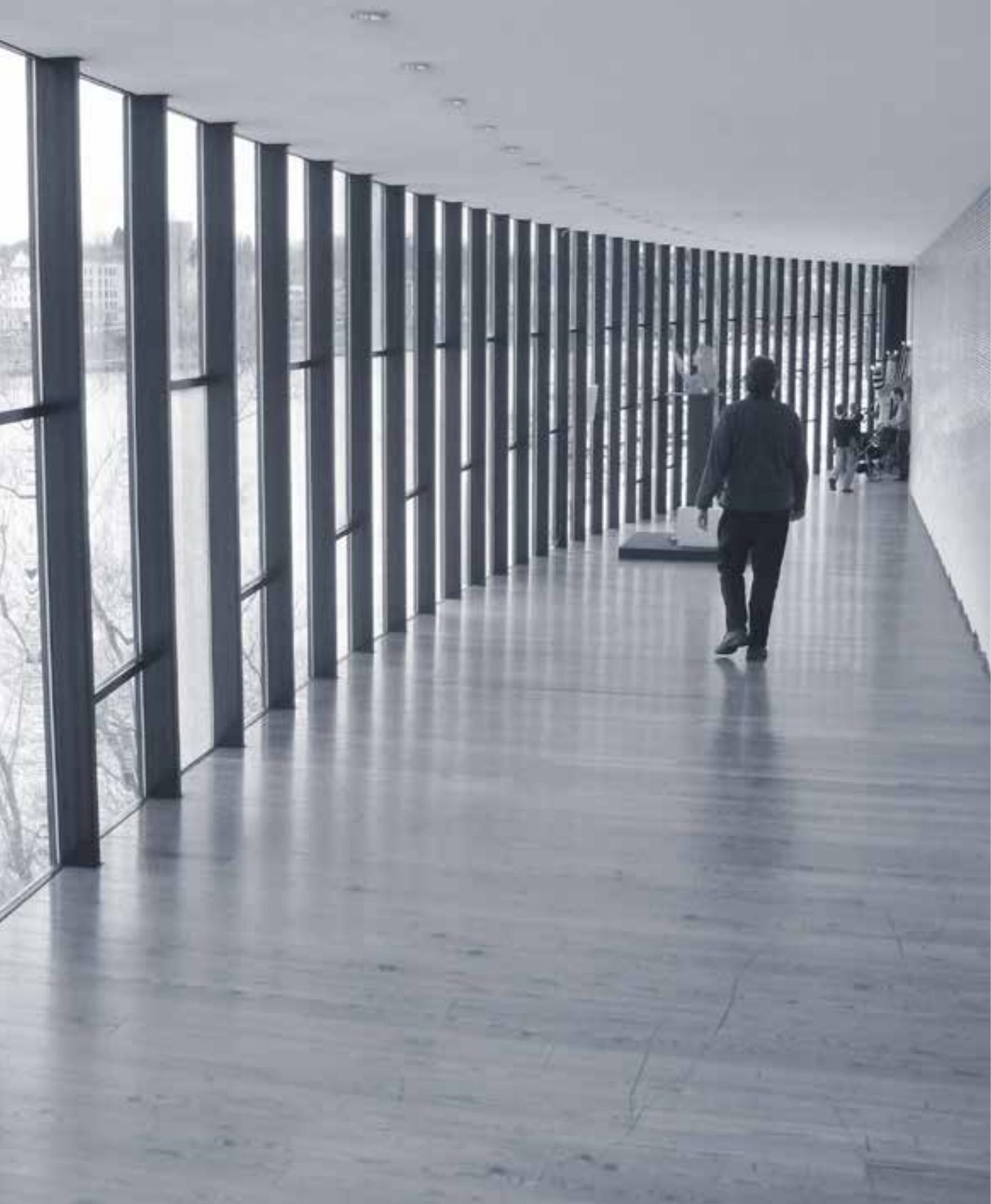




\section{Grundlagen des Rechts}

\subsection{Die Funktion des Rechts im ärztlichen Alltag}

Eine der wichtigsten Funktionen des Rechts besteht darin, das Zusammenleben der Menschen zu ordnen. Mit dem Recht sollen das Verhalten der Menschen, ihre Einstellung und Erwartung so gesteuert werden, dass Konflikte möglichst vermieden werden.

Im medizinischen Alltag müssen zahlreiche Entscheidungen getroffen werden, die auf einer Abwägung verschiedener, sich teilweise entgegenstehender Interessen oder Ziele beruhen. Beispielsweise besteht ein Zielkonflikt bei der Aufklärung des Patienten über Operationsrisiken: Einerseits hat die Patientin ein Interesse an einer umfassenden Kenntnis aller bekannten Gefahren; andererseits soll sie nicht so sehr verängstigt werden, dass sie sich gegen einen grundsätzlich notwendigen Eingriff entscheidet.

Während in einigen dieser Situationen dem Arzt und der Ärztin ein weites Ermessen eingeräumt wird, sind in anderen Fällen Rechtsnormen vorhanden, die die Entscheidung vorgeben oder zumindest wesentlich steuern. Gewisse Entscheidungen von grosser Tragweite sollen und können weder ausschliesslich von den Betroffenen selbst noch von den beteiligten Medizinalpersonen getroffenen werden.

Hinzu kommt, dass gerade das Medizinrecht einen Lebensbereich betrifft, der in der Bevölkerung mit einer erhöhten Sensibilität wahrgenommen wird. Die zugrunde liegenden ethischen und gesellschaftspolitischen Fragen sind umstritten (z.B. Abtreibung, Sterbehilfe, Zwangsmedikation); gleichzeitig stehen zentrale Rechtsgüter wie Leben, Gesundheit oder persönliche Freiheit auf dem Spiel. Daher besteht in der Praxis das Bedürfnis nach klaren Leitlinien, die dabei helfen, das eigene Verhalten auszurichten. Zudem soll der Einzelne zumindest teilweise von der Verantwortung entbunden werden, solche Entscheidungen im Alleingang treffen zu müssen. Es ist deshalb Sache der Rechtsordnung, diese Ziel- oder Interessenkonflikte - gestützt auf das Ergebnis einer vorgängig vorgenommen Wertung und einer politischen Diskussion - zu regeln.

Die Schwierigkeit besteht nun allerdings darin, dass Rechtsnormen das Gleichgewicht suchen müssen zwischen abstrakten Regeln und konkreten Regeln für den spezifischen Einzelfall. Der Gesetzgeber beschränkt sich in der Regel auf den Erlass von allgemeinen Normen und vertraut darauf, dass die Behörden und Bürger passende Anwendungen finden. Dies heisst aber auch: Wer jemals mit dem Recht zu tun gehabt hat, weiss, wie viel Unschärfe mit zahlreichen rechtlichen Normen verbunden ist, und wie schwierig es ist, einen Juristen zu einer klaren und abschliessenden Aussage zu einer Rechtsfrage zu bewegen. 
Prognosen über die rechtliche Beurteilung eines Verhaltens sind in vielen Einzelfällen nicht möglich oder wenigstens nicht eindeutig; was Recht ist, stellt sich daher manchmal erst im Nachhinein heraus. Unter diesem Gesichtspunkt ist das Recht nicht nur Leitlinie im Rahmen der praktischen Tätigkeit des Arztes, die es ihm ermöglicht, die Konsequenzen des eigenen Verhaltens abzusehen, sondern erscheint auch gleichzeitig als Damoklesschwert über den Köpfen der handelnden Personen. Sie müssen sich ständig die Frage stellen, wie eine hypothetische Richterin nachträglich über ihr Verhalten entscheiden würde. Die massgeblichen Normen sollten hingegen zumindest im Strafrecht so bestimmt sein, dass sie die Folgen des eigenen Verhaltens möglichst als vorhersehbar erscheinen lassen.

\subsection{Stufen des Rechts}

Die gesetzlichen Regelungen sind hierarchisch aufgebaut und werden meist konkreter, je weiter unten sie in der Hierarchie eingereiht sind.

\section{Verfassung}

Auf der obersten Stufe steht in der Schweiz das Verfassungsrecht, d.h. das Recht der Bundesverfassung und der einzelnen Kantonsverfassungen. Auch wenn es sich dabei meist nur um relativ allgemein gehaltene Bestimmungen handelt, bilden diese Artikel die Basis und den Ausgangspunkt für die gesamte Rechtsordnung. Aus der Sicht der Patientin von grosser Bedeutung ist vor allem das im Grundrechtskatalog der Bundesverfassung und in der Europäischen Menschenrechtskonvention garantierte Recht auf Leben und persönliche Freiheit. Das Recht auf Leben schützt die Gesamtheit der physischen und psychischen Funktionen in umfassender Weise. Das Grundrecht der persönlichen Freiheit beinhaltet das Recht, über den eigenen Körper zu bestimmen und keine körperlichen Eingriffe hinnehmen zu müssen. Im Weiteren garantiert das Recht auch die psychische Unversehrtheit sowie die Bewegungsfreiheit, die etwa bei der Zwangseinweisung in Anstalten tangiert sein kann.

Im Gegensatz zum Recht auf Leben kann das Recht auf persönliche Freiheit aber eingeschränkt werden - Beispiele sind die zwangsweise Blutentnahme oder die medikamentöse Zwangsbehandlung -, sofern dafür eine gesetzliche Grundlage vorhanden ist, ein öffentliches Interesse vorliegt und der Eingriff verhältnismässig ist.

Die Bundesverfassung enthält ausserdem eine Reihe von sogenannten Kompetenznormen. Dadurch wird die Zuständigkeit des Bundesgesetzgebers begründet, etwa um eine Kranken- und Unfallversicherung zu schaffen, die Gesundheit zu schützen, den Umgang mit Lebensmitteln oder Heilmitteln zu regeln, übertragbare Krankheiten zu bekämpfen oder Gesetze im Bereich der Humanforschung, der Fortpflanzungs- und der Transplantationsmedizin zu erlassen. Alle diese Bereiche werden durch spezielle Bundesgesetze weiter ausgeführt. 
Faktisch ebenfalls Verfassungsrang haben Staatsverträge wie insbesondere die bilateralen Abkommen mit der EU.

\section{Gesetze und Verordnungen}

Auf der nächsttieferen Stufe des Gesetzesrechts findet sich eine Reihe von Spezialgesetzen. Während langer Zeit gab es nur vereinzelte Regelungen, die speziell auf die ärztliche Tätigkeit zugeschnitten waren. Es handelte sich dabei um Lebensbereiche, die als so bedeutsam empfunden wurden, dass auf eine Normierung nicht verzichtet werden konnte, beispielsweise die Abtreibungsfrage oder die Regelung des Arztgeheimnisses im Strafgesetzbuch. Ebenfalls auf Gesetzesstufe ist seit längerer Zeit das Sozialversicherungsrecht geregelt, wobei für den medizinischen Alltag insbesondere das Krankenversicherungsgesetz von Bedeutung ist. Dieses Gesetz erfuhr 2007 eine weitreichende Teilrevision, mit der ein neues System der Spitalfinanzierung in Form von Fallpauschalen (DRG) und fixer Abgeltung der Spitalleistungsaufträge durch Kantone und Versicherer eingeführt wurde. Die Revision trat 2009 in Kraft und wird von den Kantonen zurzeit umgesetzt.

In den letzten Jahren hat der Bund eine Reihe von Spezialgesetzen erlassen, die sich spezifisch an die Medizinalpersonen richten. So wurden das Fortpflanzungsmedizingesetz (2002), das Sterilisationsgesetz (2005), das Transplantationsgesetz (2007) sowie das Bundesgesetz über genetische Untersuchungen (2007) in Kraft gesetzt. Eine umfassende Neuregelung hat zudem das Humanforschungsrecht erfahren. 2010 nahmen Volk und Stände einen neuen Verfassungsartikel über die Forschung am Menschen an; dieser Artikel wird durch das Bundesgesetz über die Forschung am Menschen (Humanforschungsgesetz, HFG) umgesetzt, das vom Parlament Ende 2011 verabschiedet wurde und voraussichtlich im Herbst 2013 in Kraft treten wird. Im Bereich der Sterbehilfe entschied demgegenüber der Bundesrat, trotz verschiedener parlamentarischer Vorstösse auf eine ausdrückliche Regelung insbesondere der organisierten Suizidhilfe im Strafrecht zu verzichten. Für die medizinische Praxis sind zudem zahlreiche Gesetzesbestimmungen relevant, die einen allgemeinen Anwendungsbereich haben. $\mathrm{Zu}$ nennen sind hier vor allem:

- die Bestimmungen des Vormundschaftsrechts bzw. des neuen Erwachsenenschutzrechts, das per 1. Januar 2013 in Kraft tritt und insbesondere die Patientenverfügung, die Vertretung bei medizinischen Massnahmen und die fürsorgerische Unterbringung neu regelt (vgl. Kap. 3.);

- die Bestimmungen des Obligationenrechts über den allgemeinen Auftrag, die auf den Behandlungsvertrag angewandt werden;

- die Regeln des allgemeinen Haftpflichtrechts, welche die Voraussetzungen und den Umfang von Schadenersatzansprüchen gegenüber Medizinalpersonen festlegen; 
- die strafrechtlichen Bestimmungen, namentlich die Delikte gegen Leib und Leben (z.B. Körperverletzung, Tötung und Beihilfe zum Selbstmord) und die strafbaren Handlungen gegen Berufspflichten (vor allem Verletzung des Berufsgeheimnisses);

- das Bundesgesetz über den unlauteren Wettbewerb.

Für das Medizinrecht ebenfalls zentral sind das Heilmittelgesetz (2002), das Epidemiengesetz und das Medizinalberufegesetz (2007). Ausserdem finden sich in vielen kantonalen Gesetzen Regelungen, die sich spezifisch an Medizinalpersonen richten, wie namentlich Bestimmungen zur Selbstdispensation, zur Spitalorganisation, zum Datenschutz oder zur Zulassung nichtuniversitärer Medizinalberufe. Diese unterscheiden sich von Kanton zu Kanton.

Die gesetzlichen Regelungen werden durch eine Vielzahl von verbindlichen eidgenössischen und kantonalen Verordnungen weiter konkretisiert und ausgeführt.

\subsection{Die privaten Regelwerke}

In der Medizin hat neben den rechtlich verbindlichen Regelungen auch das sogenannte "soft law» eine grosse Bedeutung. Darunter versteht man nichtstaatliche Regeln. Sie binden zwar den staatlichen Richter nicht; in der Praxis können sie aber starken Einfluss auf Rechtsprechung und Gesetzgebung haben.

\section{Standesrecht der FMH}

Im Medizinrecht ist vor allem das Standesrecht wichtig: In der Schweiz gehört die überwiegende Zahl von Ärztinnen und Ärzten der Berufsorganisation FMH an. Diese hat eine Standesordnung erlassen, in der viele Grundsätze der ärztlichen Tätigkeit geregelt werden, wenn auch meist nur in allgemeiner Form (vgl. Kap. 2.4.).

\section{Medizin-ethische Richtlinien der SAMW}

Auch die medizin-ethischen Richtlinien der Schweizerischen Akademie der Medizinischen Wissenschaften (SAMW) sind von grosser Bedeutung. Im Anschluss an die erste Herztransplantation wurde bereits im Jahre 1969 die «Richtlinie für die Definition und Diagnose des Todes» veröffentlicht, die in kurzer Zeit zum unverzichtbaren Instrument in der ärztlichen Praxis geworden ist und der von allen Seiten Anerkennung zuteil wurde. Gestützt auf diesen Erfolg sah sich die Akademie veranlasst, weitere Richtlinien zu diversen Themenkomplexen zu erstellen; diese Richtlinien decken heute beinahe die gesamte Bioethikdiskussion ab. Ziel dieser Richtlinien ist es, den praktisch tätigen Arzt bei der täglichen Arbeit zu unterstützen. Es soll - beruhend auf der geltenden Rechtslage - ein verlässlicher, leicht zugänglicher Standard geschaffen werden; gleichzeitig werden Probleme und Abläufe offen gelegt und dadurch eine öffentliche Diskussion ermöglicht. 
Trotz der grossen Bekanntheit dieser Richtlinien und ihres erheblichen Einflusses auf die Praxis darf nicht vergessen werden, dass ihr Erlass durch die SAMW keine staatliche Regelung mit formeller Bindungswirkung ist. Sie können deshalb nicht zwangsweise durchgesetzt werden, und ihre Verletzung zieht nicht, wie bei der Verletzung von staatlichem Recht, eine staatlich vorgesehene Sanktion nach sich. Eine wichtige Rolle spielen sie aber in der Rechtsauslegung, indem sie beispielsweise vom Bundesgericht häufig als Massstab für den Stand der medizinischen Wissenschaften herangezogen werden.

Indem allerdings die FMH praktisch alle Richtlinien der SAMW in die Standesordnung aufnimmt, werden sie auf die Stufe des Standesrechts erhoben und erhalten so für die überwiegende Zahl von Ärzten unmittelbare standesinterne, also vereinsrechtliche, Verbindlichkeit. Zudem haben verschiedene Kantone einzelne Richtlinien auf dem Wege des Gesetzesrechts für verbindlich erklärt; die Richtlinien werden auf diese Weise auf die Stufe von formellen Gesetzen erhoben. Ihnen kommt damit innerhalb des Kantons die gleiche Verbindlichkeit zu wie allen anderen Gesetzen.

\section{Klinische Guidelines}

Zu den privaten Regelwerken gehören auch Richtlinien, wie sie etwa in klinischen Guidelines für einzelne Indikationen formuliert sind. Sie beschreiben den aktuellen Stand der Wissenschaft und sollen den Arzt dabei unterstützen, dem Patienten die bestmögliche Therapie vorzuschlagen. Solche Guidelines können bei der rechtlichen Beurteilung eines Falls herangezogen werden, um abzuklären, was dem üblichen Standard der medizinischen Behandlung gemäss aktuellem Stand der Wissenschaften entspricht. Klinische Guidelines waren bisher im Haftpflichtrecht nicht relevant, haben hingegen eine Bedeutung im Sozialversicherungsrecht, wo die Rechtssicherheit einen gewissen Schematismus fördert.

\subsection{Neue Entwicklungen}

In den letzten Jahren wurde eine Reihe von Gesetzen geschaffen, die sich mit neuen Entwicklungen in der Medizin befassen. Weitere Gesetzesentwürfe und Gesetzgebungsprojekte, die einen direkten Einfluss auf den medizinischen Alltag haben dürften, sind in Bearbeitung.

Während langer Zeit schien es so, als halte sich der Gesetzgeber bewusst von den medizinrechtlichen Themen fern. Viele neuere Entwicklungen der Medizin wurden vom Gesetzgeber weitgehend ignoriert, obwohl die bestehende Rechtsordnung mit ihrem herkömmlichen Instrumentarium die neuen Fragen nicht mehr befriedigend beantworten konnte. Die Richtlinien der SAMW waren deshalb trotz ihrer fehlenden staatlichen Legitimation während langer Zeit die einzige Orientierungshilfe für den Arzt und die Ärztin. 
Erst in den letzten Jahren hat der Gesetzgeber eine aktivere Rolle eingenommen und ist seiner Aufgabe, für Rechtssicherheit zu sorgen, wieder nachgekommen. In den letzten Jahren wurden denn auch Gesetze geschaffen, um genetische Untersuchungen von Menschen, die Fortpflanzungsmedizin, die Transplantation, die Sterilisation und die Humanforschung spezifisch zu regeln. Das Humanforschungsgesetz wird zusammen mit dem sich noch in Bearbeitung befindlichen Verordnungsrecht voraussichtlich 2014 in Kraft treten, während sich das Transplantationsgesetz bereits in der ersten Teilrevision befindet.

Ebenfalls revidiert werden zurzeit das Medizinalberufe- und das Epidemiengesetz. Im Zentrum der Revision des Medizinalberufegesetzes stehen die bessere Verankerung der Grundversorgung sowie die Aufnahme der Komplementärmedizin bei der Aus- und Weiterbildung. Die Totalrevision des Epidemiengesetzes soll eine zeitgemässe Erkennung, Überwachung, Verhütung und Bekämpfung von übertragbaren Krankheiten gewährleisten. Im Bereich des Krankenversicherungsrechts ist für die medizinische Praxis insbesondere die gegenwärtig laufende Umsetzung der neuen Spitalfinanzierung ${ }^{1}$ von Bedeutung. Im Juni 2012 wurde die Managed-Care-Vorlage deutlich abgelehnt (vgl. zu den Folgen Kap. 4.12.). Im Rahmen seiner eHealth-Strategie entwickelte der Bund einen Vorentwurf für ein Bundesgesetz über das elektronische Patientendossier, das eine bessere Qualität der Behandlungsprozesse, eine höhere Patientensicherheit sowie mehr Effizienz im Gesundheitswesen unterstützen soll. Des Weiteren beabsichtigt der Bundesrat, eine gesamtschweizerisch einheitliche Rechtsgrundlage für die Registrierung von Krebs und weiteren stark verbreiteten oder bösartigen Krankheiten zu schaffen. Aktuell beschäftigt das Parlament zudem die Volksinitiative «Ja zur Hausarztmedizin» mit ihrer Forderung nach einem Verfassungsartikel zur Förderung der Hausarztmedizin und mit der Wiedereinführung des Zulassungsstopps für Spezialisten.

Trotz der vielen neuen Gesetze bleiben auch die Richtlinien der SAMW und das Standesrecht der FMH für das Medizinrecht wichtig, vor allem dort, wo sich der Gesetzgeber noch nicht zu einer eigenen Lösung durchringen konnte oder diese sehr allgemein gehalten ist.

$1 \mathrm{FMH}$ und $\mathrm{H}+$ lassen mögliche Auswirkungen der Einführung von SwissDRG auf die Ärzteschaft im Rahmen von Begleitstudien untersuchen. Aktuelle Ergebnisse der Studien sind einsehbar unter: www.fmh.ch/tarife/begleitforschung.html [Stand: 17.4.2012]. 


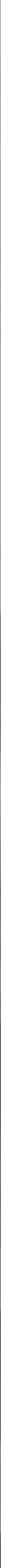




\section{Rahmenbedingungen der ärztlichen Tätigkeit}

\subsection{Aus-, Weiter- und Fortbildung}

Das Medizinalberufegesetz (MedBG) bildet die Grundlage für die Aus-, Weiterund Fortbildung von Ärztinnen und Ärzten. Es regelt auch die Berufsausübung in der Schweiz und die Führung des Medizinalberuferegisters (MedReg). Das MedBG ist seit September 2007 in Kraft und wird zurzeit erstmals revidiert.

\section{Aus- und Weiterbildung}

Wer in der Schweiz Medizin studieren möchte, kann das im Bologna-System aufgebaute sechsjährige Bachelor-/Master-Studium an den fünf Medizinischen Fakultäten Basel, Bern, Genf, Lausanne und Zürich absolvieren; der Abschluss als Bachelor of Medicine (BMed) ist in Fribourg möglich, in Neuchâtel kann das erste Studienjahr absolviert werden. An verschiedenen Fakultäten müssen Bewerberinnen und Bewerber vorgängig einen Eignungstest bestehen (Numerus clausus). Wer das Studium mit dem Master of Medicine (MMed) abschliesst und die eidgenössische Prüfung besteht, erhält das eidgenössische Arztdiplom. Das Arztdiplom berechtigt allerdings nur zur unselbstständigen Tätigkeit in einem Spital oder einer Arztpraxis.

Nach Erwerb des eidgenössischen Arztdiploms beginnt die Phase der Weiterbildung, die mit einem eidgenössischen Weiterbildungstitel abgeschlossen wird. Ein Weiterbildungstitel ist Voraussetzung für die Aufnahme einer selbstständigen Tätigkeit und damit für die Eröffnung einer Arztpraxis (vgl. Kap. 10.2.).

Das Schweizerische Institut für ärztliche Weiter- und Fortbildung (SIWF), seit 2009 ein eigenständiges Organ der FMH, regelt und organisiert die Weiterbildung im Auftrag des Bundes. Grundlage bilden dabei die Weiterbildungsordnung (WBO) und die 44 vom Eidgenössischen Departement des Innern akkreditierten Weiterbildungsprogramme. ${ }^{2}$ Jedes Weiterbildungsprogramm umschreibt detailliert die Dauer und die Anforderungen für den entsprechenden Facharzttitel. Operative Fächer setzen beispielsweise das Durchführen einer bestimmten Anzahl Operationen voraus. In den meisten Weiterbildungsprogrammen sind theoretische Weiter- und Fortbildungsveranstaltungen gefordert, teilweise auch der Nachweis von wissenschaftlichen Publikationen. 
$\mathrm{Zu}$ Beginn einer Weiterbildungsperiode legen Weiterbildner und Weiterzubildende jeweils in einem Weiterbildungsvertrag gemeinsam die zu erreichenden Ziele fest. Sie führen zu deren Überprüfung regelmässige Standortgespräche und, insbesondere am Ende einer Weiterbildungsperiode, ein Evaluationsgespräch durch. Die absolvierte Weiterbildung sowie die Lernfortschritte werden laufend in einem für jedes Fachgebiet separat erstellten e-Logbuch festgehalten. ${ }^{3}$ Die Facharztweiterbildung wird an dafür anerkannten Spitälern und Kliniken durchgeführt, die in ein Zertifizierungssystem eingebunden sind. Es ist davon auszugehen, dass die Listen der anerkannten Weiterbildungsstätten sowie das e-Logbuch ab 2013 für die Finanzierung der strukturierten Weiterbildung durch die Kantone eine massgebende Rolle spielen werden.

Auf dem Weg zu einem Grundversorgertitel (Allgemeine Innere Medizin, Kinderund Jugendmedizin, Praktischer Arzt) besteht ein grosses Bedürfnis nach praxisnaher Weiterbildung. Es sind Bestrebungen seitens der betroffenen Berufskreise, der Konferenz der Kantonalen Gesundheitsdirektoren (GDK) und des Bundes im Gang, die Praxisassistenz im Bereich der Grundversorgung für alle Kandidaten eines Grundversorger-Facharzttitels zugänglich zu machen.

Neben den eidgenössischen Weiterbildungstiteln verleiht das SIWF zusätzlich Schwerpunkte und Fähigkeitsausweise, die eine Spezialisierung in einem bestimmten Fachgebiet dokumentieren. Sie spielen für die Qualitätssicherung und teilweise auch für die Abrechnung von Leistungen zu Lasten der Sozialversicherer eine wichtige Rolle.

\section{Bilaterale Verträge Schweiz - EU}

Alle in der EU-Richtlinie 2005/36 aufgeführten Facharzttitel sind in den jeweiligen Ländern Europas gegenseitig anerkannt und müssen den Minimalstandards der Richtlinie entsprechen. Zwischen der Schweiz und der EU besteht seit 2002 mit dem Freizügigkeitsabkommen ein Staatsvertrag über die gegenseitige Anerkennung von Berufsqualifikationen. Das Abkommen verweist in seinem Anhang III auf die für die Schweiz anwendbare EU-Richtlinie und listet die eidgenössischen Facharzttitel entsprechend den Fachgebietsrubriken der EU-Richtlinie auf. Das Bundesamt für Gesundheit bzw. dessen Medizinalberufekommission (MEBEKO) muss gestützt auf den Staatsvertrag die in der Richtlinie aufgeführten Diplome und Facharzttitel automatisch, d.h. ohne Prüfung des konkret absolvierten Weiterbildungscurriculums, anerkennen. Das Gleiche gilt auch für die Anerkennung von eidgenössischen Diplomen und Titeln in den Mitgliedstaaten 
der EU. Mangels eines entsprechenden Staatsvertrags sind die ausserhalb der EU erworbenen Berufsqualifikationen grundsätzlich nicht direkt und nur unter klar definierten Bedingungen gegebenenfalls indirekt über einen Mitgliedstaat der EU anerkennbar. ${ }^{4}$

Ausländische Ärztinnen und Ärzte, die in der Schweiz einen eidgenössischen Facharzttitel erwerben möchten, müssen sich in jedem Fall über ein von der MEBEKO formell anerkanntes ausländisches Arztdiplom ausweisen oder gegebenenfalls das eidgenössische Staatsexamen absolvieren.

Sämtliche Ärztinnen und Ärzte, die einen eidgenössischen oder anerkannten ausländischen Weiterbildungstitel besitzen, sind im öffentlich zugänglichen Ärzteverzeichnis der FMH und im Medizinalberuferegister des Bundes aufgeführt. ${ }^{5}$

\section{Fortbildung}

Das MedBG verlangt von jedem Inhaber eines Weiterbildungstitels, dass er sich während seiner ärztlichen Berufstätigkeit permanent fortbildet. Aufsichtsbehörde für den allfälligen Nachweis einer angemessenen Fortbildung ist die kantonale Gesundheitsdirektion. Eine Verletzung dieser Berufspflicht kann einen Verweis oder eine Busse nach sich ziehen.

Daneben müssen Ärztinnen und Ärzte auch im Rahmen von TARMED für die sogenannten Besitzstandspositionen eine entsprechende Fortbildung nachweisen, damit die Krankenversicherer ihrer diesbezüglichen Leistungspflicht nachkommen.

Das SIWF bietet gemeinsam mit den 44 Fachgesellschaften im Sinne einer Dienstleistung ein Fortbildungsdiplom an, das Gesundheitsbehörden und Krankenversicherer anerkennen. Über die elektronische Fortbildungsplattform des SIWF lässt sich das Fortbildungsdiplom einfach und bequem ausdrucken. Dieses Vorgehen bietet den zusätzlichen Vorteil, dass das Diplom automatisch im Ärzteverzeichnis www.doctorfmh.ch erscheint und gleichzeitig alle Besitzstandspositionen validiert bleiben, die damit weiterhin abgerechnet werden dürfen.

Die Fortbildungsordnung (FBO) des SIWF geht von einer Fortbildung von durchschnittlich 80 Stunden jährlich aus. Die einzelnen Fortbildungsprogramme werden durch die jeweiligen Fachgesellschaften ausgearbeitet und umgesetzt. ${ }^{6}$ 
Die in der FBO verankerte Richtlinie «Zusammenarbeit Ärzteschaft - Industrie» der SAMW verlangt für den Kongressbesuch einen Selbstkostenbeitrag der Ärztin oder des Arztes. Für Fortbildungen sollen mehrere Sponsoren gefunden werden, da bei Monosponsoring ein grösseres Risiko für Abhängigkeiten besteht. Die Richtlinie stellt auch Kriterien für die Anerkennung von Fortbildungen durch die Fachgesellschaften auf: So sollen Inhalt und Ablauf der Veranstaltung durch die ärztlichen Fachgremien (mit)bestimmt werden. Die Zusammenarbeit mit den Industriepartnern soll in einem schriftlichen Vertrag festgehalten werden.

Die FMH hat diese SAMW-Richtlinie ins Standesrecht und das SIWF hat die wichtigsten Punkte in die Fortbildungsordnung (FBO) übernommen. Damit ist die Richtlinie für FMH-Mitglieder bindend.

\subsection{Das Medizinalberufegister}

Des Medizinalberuferegister (MedReg) enthält bisher nur die Angehörigen von Medizinalberufen (Ärzte, Apotheker, Zahnärzte, Tierärzte), die entweder ein Schweizer Arztdiplom oder einen Schweizer Weiterbildungstitel, oder aber ein vom Bund formell anerkanntes äusländisches Arztdiplom oder einen formell anerkannten ausländischen Weiterbildungstitel besitzen. ${ }^{7}$ Aus Sicht der FMH sollte das MedReg alle in der Schweiz als Ärztin oder Arzt in der Patientenbehandlung tätigen Personen erfassen, also auch diejenigen ohne formell anerkanntes Diplom, denn es sollte zum Schutz des Patienten allfällige Disziplinarmassnahmen betreffend alle Ärzte erfassen können.

7 «Das Medizinalberuferegister enthält Daten betreffend die Inhaberinnen und Inhaber von Diplomen und Weiterbildungstiteln gemäss MedBG», Art. 2 Registerverordnung MedBG. 


\subsection{Die Berufs- und Kassenzulassung}

Obschon die Berufszulassung zur selbstständigen ärztlichen Tätigkeit im MedBG gesamtschweizerisch geregelt ist, sind die Kantone für die Umsetzung wie auch für die Berufsaufsicht zuständig. Wer über ein schweizerisches oder anerkanntes Arztdiplom und einen schweizerischen oder anerkannten Weiterbildungstitel verfügt, einen guten Leumund hat und gesund ist, hat einen Rechtsanspruch auf die Erteilung der kantonalen Berufsausübungsbewilligung. Weitere fachliche Qualifikationen dürfen die Kantone nicht fordern (vgl. Kap. 10.2.). Für Spezialisten und auch für Grundversorger mit zusätzlichem Spezialistentitel wird ab 1. April 2013 voraussichtlich wieder ein Zulassungsstopp gelten und damit zusätzlich zur kantonalen Berufsausübungsbewilligung eine KVG-Zulassung nötig sein, um den KVGTarifverträgen beizutreten und Kassenpatienten zu behandeln (vgl. Kap. 10.2. zu KVG-Revisionen).

Die Bedingungen für die Aufnahme einer unselbstständigen Tätigkeit sind in den kantonalen Gesetzen geregelt.

\subsection{Berufspflichten}

Sowohl das Medizinalberufegesetz als auch die Standesordnung der FMH legen die Berufspflichten eines Arztes oder einer Ärztin fest. Dazu gehören auch die Nothilfepflicht und die Pflicht, im organisierten Notfalldienst mitzuwirken.

Das Medizinalberufegesetz (MedBG) regelt in Artikel 40 einige zentrale Berufspflichten für selbstständig tätige Ärztinnen und Ärzte: Sie müssen ihren Beruf sorgfältig und gewissenhaft ausüben, bilden sich lebenslang fort, wahren die Patientenrechte, machen nur objektive und einem öffentlichen Bedürfnis entsprechende Werbung, handeln bei der Zusammenarbeit mit Angehörigen anderer Gesundheitsberufe unabhängig von finanziellen Vorteilen, wahren das Berufsgeheimnis, leisten in dringenden Fällen Beistand und wirken nach Massgabe der kantonalen Vorschriften in Notfalldiensten mit. Zu den Berufspflichten gehört auch, dass Ärztinnen und Ärzte eine angemessene Haftpflichtversicherung haben oder für andere, gleichwertige Sicherheiten sorgen. Die kantonale Gesundheitsdirektion überwacht, ob diese Berufspflichten eingehalten werden.

Die FMH-Standesordnung gilt für alle FMH-Mitglieder ${ }^{8}$ unabhängig von ihrer beruflichen Stellung. Sie konkretisiert die wichtigsten Berufspflichten des MedBG sowie zusätzliche wichtige berufsethische Regeln. Zu den zusätzlichen Regeln gehören beispielsweise die freie Arztwahl, Regeln zur Annahme und Ablehnung des 
Behandlungsauftrags, der Umgang mit umstrittenen Heilverfahren, die Transparenz bei Gutachten, die Aufklärungspflicht, Werbung und Medientätigkeit, Betreuung von Sportlern oder die KG-Führung. Auch die meisten medizin-ethischen Richtlinien der SAMW sind durch Aufnahme in die Standesordnung Teil des ärztlichen Standesrechts geworden.

Die Standesordnung enthält zudem Bestimmungen über die kollegiale Zusammenarbeit: So sollen sich Kollegen gegenseitig über Befunde und Behandlung informieren, wenn sie gemeinsam an einer Behandlung beteiligt sind und soweit das Einverständnis des Patienten vorliegt. Nötige Kritik soll sachlich geäussert werden.

Verstösse gegen die FMH-Standesordnung werden auf Anzeige erstinstanzlich durch die Standeskommission der kantonalen Ärztegesellschaft bzw. des VSAO oder des VLSS geahndet. ${ }^{9}$ Auch der staatliche Richter zieht Bestimmungen des FMH-Standesrechts zur Interpretation der Berufspflichten gemäss MedBG heran, soweit sie ihm angemessen erscheinen. ${ }^{10}$

\section{Nothilfepflicht}

Wer «einem Menschen, der in unmittelbarer Lebensgefahr schwebt, nicht hilft, obwohl es ihm den Umständen nach zugemutet werden könnte», macht sich strafbar. ${ }^{11}$ Wer beim Wandern im Gebirge auf einen Verletzten am Wegrand stösst, ist nothilfepflichtig. Massgeblich für das Zumutbare sind unter anderem die Kenntnisse und Fertigkeiten zur Nothilfe. Die Nothilfepflicht der Ärztinnen und Ärzte geht somit weiter als die des Laien. Diese allgemeine Nothilfepflicht hängt nicht davon ab, ob die Ärztin oder der Arzt «im Dienst» ist oder nicht. Es kommt auch nicht darauf an, ob sie eine Berufsausübungsbewilligung haben. Selbstverständlich muss auch bei der Nothilfe sorgfältig gehandelt werden. In der Schweiz musste aber bisher niemand Angst haben vor Schadenersatzforderungen wegen des Vorwurfs, er habe falsche Nothilfe geleistet. Weil es sich um eine allgemeine Bürgerpflicht handelt, wäre ein Behandlungsfehler von der privaten Haftpflichtversicherung zu übernehmen - die Ärztin braucht wegen der Nothilfepflicht keine eigene Berufshaftpflichtversicherung.

9 Die Zuständigkeit hängt davon ab, wo der Arzt oder die Ärztin Basismitglied ist.

10 So hat das Verwaltungsgericht des Kantons Waadt am 15. Mai 2007 einen Arzt trotz EPO-Abgabe freigesprochen, weil die FMH-Standesordnung vor 2001 noch keine explizite Antidoping-Bestimmung enthielt.

11 Art. 128 StGB, Unterlassung der Nothilfe: Wer einem Menschen, den er verletzt hat, oder einem Menschen, der in unmittelbarer Lebensgefahr schwebt, nicht hilft, obwohl es ihm den Umständen nach zugemutet werden könnte, wer andere davon abhält, Nothilfe zu leisten, oder sie dabei behindert, wird mit Freiheitsstrafe bis drei Jahre oder Geldstrafe bestraft. 
Seit Einführung dieser Bestimmung im Jahr 1990 gab es nur wenige Gerichtsfälle, die sich mit unterlassener Nothilfe befassten. So entschied das Bundesgericht im Jahr 1995, dass unmittelbare Lebensgefahr dann gegeben ist, «wenn jemand nach dem Konsum einer Überdosis Heroin Gefahr läuft, in einigen Stunden zu sterben. Die Hilfeleistungspflicht besteht für jeden, der sich in der Wohnung der gefährdeten Person befindet; aufgrund der Umstände genügte es, telefonisch medizinische Hilfe zu holen.» (BGE 121 IV 18). Bekannt geworden ist auch der Freispruch eines praktizierenden Spezialisten, der die Behandlung seines Patienten in der Arztpraxis fortsetzte, obschon jemand in der Nähe der Praxis auf dem Trottoir lag und in Not war. Der Richter begründete den Freispruch mit dem funktionierenden örtlichen Notfalldienst der Ärzteschaft.

\section{Notfalldienst}

Praktizierende Ärztinnen und Ärzte müssen am organisierten Notfalldienst teilnehmen. Dazu verpflichten sie das MedBG und die kantonalen Gesundheitsgesetze. Die Kantone haben die Organisation eines Notfalldienstes an die jeweilige kantonale Ärztegesellschaft delegiert. Diese kann einzelne Ärzte vom Notfalldienst befreien. Gemäss Bundesgericht ist der Notfalldienst ein öffentlicher Dienst, für dessen Organisation öffentliches Recht gilt; deshalb gehören beispielsweise Kriterien für Ersatzabgaben im formellen Gesetz geregelt. ${ }^{12} \mathrm{Im}$ Gegensatz zum ärztlichen Notfalldienst wird das Rettungswesen, beispielsweise die Ambulanz- und Helikopterrettung, von den Kantonen selbst organisiert oder an Dritte (Rega usw.). delegiert. Nach geltendem TARMED-Tarif wird der konkrete Einsatz im Notfalldienst - die Notfallkonsultation, der Notfallbesuch - von der Sozialversicherung bezahlt. Im Hinblick auf die Förderung der Hausarztmedizin bestehen Verbesserungsmöglichkeiten bei der Finanzierung der Notfalldienstorganisation, der Telefonzentralen und des Pikettdienstes.

\section{Rettungswesen}

Die «Plattform Rettungswesen» der FMH hat im Jahr 2001 Ziele und Kriterien für den ärztlichen Notfalldienst definiert und 2010 ein Leitbild Rettungswesen veröffentlicht. ${ }^{13}$ Diese Regeln sind zwar nicht verbindliches Standesrecht, da sie nicht von der Ärztekammer verabschiedet wurden. Aber als fachliche Richtlinien eines Expertengremiums können sie für allfällige haftpflichtrechtliche Auseinandersetzungen eine Rolle spielen. 


\subsection{Werbung und Ausschreibung}

Um sich im Gesundheitsmarkt gut zu positionieren, möchten viele Ärztinnen vermehrt mit Werbung auf sich aufmerksam machen, etwa mit eigenen Websites. Das Medizinalberufegesetz MedBG und die Standesordnung lassen Information und Werbung zu, doch gilt für beide der Grundsatz, dass Ärzte nur Werbung machen dürfen, die objektiv ist, dem öffentlichen Bedürfnis entspricht und weder irreführend noch aufdringlich ist. ${ }^{14}$ Zuständig für die Durchsetzung des MedBG ist die kantonale Gesundheitsdirektion. Für die FMH-Mitglieder enthält die FMH-Richtlinie «Information und Werbung» zusätzlich einige standesrechtliche Grundsätze für die Arztwerbung. ${ }^{15}$ Deren Umsetzung und Konkretisierung erfolgt durch die kantonalen Ärztegesellschaften, insbesondere bezüglich der Frage, wann, wie oft und wie gross in welchen Medien inseriert werden kann.

Die Ausschreibung von akademischen Titeln, fachlichen Qualifikationen, Dienstleistungen und Mitgliedschaften auf Briefschaften, im Telefonbuch usw. sind Teil der Werbung. Die zu beachtenden Regelungen für die Ausschreibung finden sich in eidgenössischen und kantonalen Gesetzen, in Universitätsgesetzen, Staatsverträgen, der FMH-Standesordnung sowie deren Richtlinie «Information und Werbung». FMH und SIWF sind daran, eine umfassende Informationsschrift mit Empfehlungen und Beispielen - v.a. auch in Bezug auf im Ausland erworbener Titel und Diplome - zu erarbeiten. Sie wird im 2013 veröffentlicht werden.

\section{Registereintragungen - «Adressbuchschwindel»}

Auch Arztpraxen werden häufiger als früher von Offerten für Einträge in verschiedenste Register und insbesondere Internetregister heimgesucht, die sehr oft mit hohen Kosten und fraglichem Nutzen verbunden sind.

Der Bund hat das Gesetz über den unlauteren Wettbewerb (UWG) revidiert und in Art. 3 Abs. 1 lit. p und q neue Unlauterkeitstatbestände definiert. Weitere Informationen auch zum Vorgehen im Falle eines ungewollt eingegangenen Vertrages mit einem Registerbetreiber sind auf der Website der FMH und dem SECO aufgeschaltet. ${ }^{16}$

14 Art. 40 lit. d MedBG.

15 Anhang 2 der FMH-Standesordnung - so darf der Arzt beispielsweise nicht mit dankbaren Patienten werben.

16 www.fmh.ch $\rightarrow$ Service $\rightarrow$ Recht $\rightarrow$ Praktische Tipps oder unter www.seco.admin.ch $\rightarrow$ unlauterer Wettbewerb $\rightarrow$ Konsumententipps. 


\title{
2.6. Sozial- und Privatversicherungen
}

\author{
Die Sozialversicherungen sind obligatorisch; daher werden Leistungen und \\ Prämien gemäss gesetzlichen Regeln festgelegt. Im Gegensatz dazu besteht bei \\ den Privatversicherungen Vertragsfreiheit.
}

\section{Sozialversicherungen}

In den letzten 80 Jahren sind in der Schweiz elf verschiedene Sozialversicherungen entstanden. Für die behandelnde Ärztin relevant sind die Kranken-, die Unfall-, die Militär- und die Invalidenversicherung. Für den berichterstattenden Arzt spielen zusätzlich die Pensionskassen und - soweit sie noch betrieben wird - die Taggeldversicherung nach KVG eine Rolle.

In den Sozialversicherungen gelten in der Regel die folgenden Grundsätze:

- Alle Bewohnerinnen und Bewohner der Schweiz müssen sich versichern. ${ }^{17}$

- Der Sozialversicherer darf keine Risikoselektion machen und muss jede Person versichern. ${ }^{18}$

- Die Leistungen werden gesetzlich definiert und erfolgen nach pauschalen Regeln.

- Es gilt öffentliches Recht und insbesondere Rechtsgleichheit. Der Versicherer muss daher vergleichbare Fälle gleich behandeln; dies gilt auch gegenüber Leistungserbringern. ${ }^{19}$

- Die Prämien werden nicht nach dem individuellen Risiko festgelegt. Es gilt das Gegenseitigkeitsprinzip, das heisst, die Versicherten bilden eine Solidargemeinschaft, die allen einen zahlbaren Zugang zur sozialen Sicherheit geben soll.

- Der Sozialversicherer hat ein gesetzliches Informationsrecht; umgekehrt darf er nur die Informationen bearbeiten, die er für seine gesetzliche Aufgabe benötigt.

Die Aufsicht erfolgt durch das BSV bzw. (für das KVG und das UVG) durch das BAG.

Seit 2003 werden die Sozialversicherungen über das Bundesgesetz über den Allgemeinen Teil des Sozialversicherungsrechts (ATSG) teilweise miteinander koordiniert. Das ATSG definiert die Rechtsbegriffe Krankheit, Unfall, Arbeitsunfähigkeit, Erwerbsunfähigkeit und Invalidität und es regelt die Streitigkeiten zwischen Versichertem und Sozialversicherer (vgl. Kap. 4.10., 4.15. und 4.16.).

18 Wiederum mit Ausnahme der Krankentaggeldversicherung gemäss KVG, Art. 69ff KVG, in der zwar niemand abgelehnt werden darf - aber der Versicherer darf während 5 Jahren Gesundheitsvorbehalte machen. Ähnliches gilt bei Freizügigkeitsfällen im überobligatorischen BVG, das aufgrund des Freizügigkeitsgesetzes von 1993 Züge einer Privat- wie auch einer Sozialversicherung hat. 


\section{Privatversicherungen}

Die Privatversicherungen unterstehen dem Bundesgesetz über den Versicherungsvertrag (VVG). Hier gilt im Gegensatz zur Sozialversicherung die Vertragsfreiheit. Dazu gehören folgende Grundsätze:

- Es besteht keine Versicherungspflicht.

- Es gibt keine Aufnahmepflicht durch den Versicherer. Dieser darf den Gesundheitszustand abklären und bei schlechten Risiken eine Versicherung verweigern, Vorbehalte machen oder höhere Prämien verlangen. Wer durch falsche Angaben zum Gesundheitszustand die Anzeigepflicht verletzt, verliert den Versicherungsschutz, wenn es sich um eine erhebliche Gefahrentatsache handelt, und der Schaden durch diese Tatsache beeinflusst wurde. Auch möglich ist die Kündigung innert einer bestimmen Frist nach Bekannt werden der Anzeigepflichtverletzung. ${ }^{20}$

- Die versicherten Leistungen werden im Versicherungsvertrag definiert (und nicht im Gesetz).

- Die Aufsicht erfolgt durch die Finanzmarktaufsicht (FINMA), im Zentrum steht die finanzielle Sicherheit der Institution.

Der Versicherer und der Versicherte sind rechtlich gleichgestellt. Es gibt keine Verfügungen und Einspracheentscheide des Versicherers. Im Streitfall muss der Versicherte beim Zivilrichter klagen. 


\section{Erwachsenenschutzrecht}

Nach langer Vorarbeit wurde das neue Erwachsenenschutzrecht (Art. 360-456 ZGB ${ }^{21}$ ) am 19. Dezember 2008 von den Eidgenössischen Räten verabschiedet und vom Bundesrat auf den 1. Januar 2013 in Kraft gesetzt. Das neue Erwachsenenschutzrecht bezweckt den Schutz von Personen, die aufgrund eines Schwächezustandes (z.B. Demenz oder psychische Störung) hilfs- und schutzbedürftig geworden sind und stellt hierfür geeignete Instrumente zur Verfügung. Mit einem Vorsorgeauftrag kann eine handlungsfähige Person ihre Betreuung und rechtliche Vertretung für den Fall ihrer Urteilsunfähigkeit festlegen. In einer Patientenverfügung legt eine urteilsfähige Person fest, welchen medizinischen Massnahmen sie im Fall ihrer Urteilsunfähigkeit zustimmt oder nicht zustimmt. Mit Hilfe dieser Instrumente kann das Selbstbestimmungsrecht auch über die Zeit der eigenen Urteilsfähigkeit hinaus gewahrt werden. Hat eine urteilsunfähige Person keine eigene Vorsorge getroffen, so räumt das neue Erwachsenenschutzrecht Angehörigen oder Nahestehenden gewisse Vertretungsrechte ein. Erweist sich schliesslich die eigene Vorsorge oder die Betreuung durch Angehörige oder Nahestehende als ungenügend, so stellen massgeschneiderte behördliche Massnahmen (Beistandschaften und fürsorgerische Unterbringung) als Ultima Ratio das Wohl und die Interessen von schutz- und hilfsbedürftigen Personen sicher.

\subsection{Eigene Vorsorge}

Um das Selbstbestimmungsrecht auch über die Zeit der eigenen Urteilsfähigkeit hinaus zu wahren, stellt das Erwachsenenschutzrecht zwei Instrumente zur Verfügung.

Mit einem Vorsorgeauftrag beauftragt jemand eine natürliche oder eine juristische Person (z.B. Pro Senectute oder eine Bank), im Fall seiner Urteilsunfähigkeit für ihn zu handeln (Art. 360 ff. ZGB). Die Aufgabenbereiche des Vorsorgebeauftragten können die Personensorge, die Vermögenssorge und/oder den Rechtsverkehr betreffen. Unter Personensorge fallen auch Anordnungen in Bezug auf allfällige medizinische Massnahmen.

Die auftraggebende Person muss zum Zeitpunkt der Errichtung des Vorsorgeauftrags handlungsfähig, d.h. urteilsfähig und volljährig sein. Wegen der grossen Tragweite des Vorsorgeauftrags ist die Errichtung an strenge Formerfordernisse geknüpft. Der Vorsorgeauftrag muss entweder öffentlich beurkundet oder eigenhändig errichtet werden. 
Mit einer Patientenverfügung (mehr dazu siehe Kap. 4.4.) kann eine urteilsfähige Person verbindlich festsetzen, welchen medizinischen Massnahmen sie im Fall ihrer Urteilsunfähigkeit zustimmt oder nicht zustimmt (Art. 370 ff. ZGB). Für die Beurteilung der Wirksamkeit einer Patientenverfügung ist in erster Linie der Arzt zuständig. Befolgt der Arzt die Anordnungen in einer Patientenverfügung nicht, so hat er die Gründe hierfür im Patientendossier festzuhalten.

\subsection{Massnahmen von Gesetzes wegen}

Das neue Erwachsenenschutzrecht räumt Angehörigen oder nahestehenden Personen eines Urteilsunfähigen gewisse gesetzliche Vertretungsrechte ein. Behördliche Massnahmen des Erwachsenenschutzes sind nur dann anzuordnen, wenn sich die eigene Vorsorge und die Betreuung durch Angehörige als ungenügend erweisen.

Gemäss neuem Erwachsenenschutzrecht kommt dem Ehegatten, der eingetragenen Partnerin oder dem eingetragenen Partner das Recht zu, den jeweiligen Partner bei Urteilsunfähigkeit in gewissen Belangen zu vertreten (gesetzliches Vertretungsrecht, Art. 374 ZGB). Sie erhalten insbesondere die Befugnis, alle zur Deckung des Unterhaltsbedarfs üblicherweise erforderlichen Rechtshandlungen vorzunehmen. Dabei muss die Erwachsenenschutzbehörde nicht eingeschaltet werden. Die Regelung erweitert die Vertretungsbefugnisse, die einem Ehegatten bereits gemäss Eherecht ${ }^{22}$ bzw. einem eingetragenen Partner gemäss Partnerschaftsgesetz ${ }^{23}$ zustehen.

Für den Fall, dass eine urteilsunfähige Person zur medizinischen Behandlung keine Anordnungen gemacht hat, legt das neue Erwachsenenschutzrecht fest, wer zur Vertretung berechtigt ist und für die urteilsunfähige Person eine Einwilligung in eine medizinische Massnahme erteilen darf (Vertretung bei medizinischen Massnahmen, Art. 378 ZGB). Vertretungsberechtigt ist an erster Stelle die in einer Patientenverfügung oder in einem Vorsorgeauftrag bezeichnete Person. Diese schliesst alle weiteren Personen von der Vertretungsmacht aus. Hat die urteilsunfähige Person keinen Stellvertreter bezeichnet, so ist an zweiter Stelle der von der Erwachsenenschutzbehörde zu diesem Zweck ernannte Beistand vertretungsberechtigt. Erst an dritter Stelle ist der Ehegatte oder der eingetragene Partner befugt, die urteilsunfähige Person zu vertreten, sofern ein gemeinsamer Haushalt besteht oder dem Urteilsunfähigen regelmässig und persönlich Beistand geleistet wird. An vierter Stelle folgt die Person, die mit der urteilsunfähigen Person einen gemeinsamen Haushalt führt und ihr regelmässig und persönlich Beistand leistet. Schliesslich sind die Nachkommen, die Eltern und zuletzt die Geschwister vertretungsberechtigt, sofern sie dem Urteilsunfähigen regelmässig und persön-

Vgl. Art. 15 PartG (Partnerschaftsgesetz; SR 211.231). 
lich Beistand leisten. Sind mehrere Personen gleichen Ranges vertretungsberechtigt, so darf der gutgläubige Arzt davon ausgehen, dass jede im Einverständnis mit den anderen handelt.

Die vertretungsberechtigte Person hat nach dem mutmasslichen Willen des Urteilsunfähigen und den objektiven Interessen über die zu treffenden medizinischen Massnahmen zu entscheiden. Während der Arzt eine in einer Patientenverfügung niedergelegte Anordnung in Bezug auf eine medizinische Massnahme unter gewissen Voraussetzungen nicht zu befolgen hat, ist er an die Entscheidung des Vertretungsberechtigten grundsätzlich gebunden. Sind allerdings die Interessen der urteilsunfähigen Person gefährdet oder nicht mehr gewahrt, so bestimmt die Erwachsenenschutzbehörde auf Antrag des Arztes oder einer anderen nahestehen Person oder von sich aus einen anderen Vertretungsberechtigten.

Der Arzt ist verpflichtet, bei der Behandlung Urteilsunfähiger einen Behandlungsplan zu erstellen und ihn den laufenden Entwicklungen regelmässig anzupassen (Art. 377 ZGB). Der Behandlungsplan ist mit der vertretungsberechtigten Person zu besprechen und zu erläutern, sodass diese in der Lage ist, ihre informierte $\mathrm{Zu}$ stimmung zur Behandlung zu erteilen. Die betroffene Person ist so weit wie möglich in die Entscheidfindung mit einzubeziehen.

\subsection{Aufenthalt in Wohn- und Pflegeeinrichtungen}

Um den Schutz urteilsunfähiger Personen in Wohn- oder Pflegeeinrichtungen zu gewährleisten, ist es gemäss neuem Recht zwingend, bei längerem Aufenthalt einen schriftlichen Betreuungsvertrag abzuschliessen (Art. 382ff ZGB). Der Vertrag regelt die Leistungen, welche die Wohn- oder Pflegeeinrichtung zu erbringen hat. Medizinische Leistungen sind nicht Inhalt des Betreuungsvertrags. Zum Vertragsabschluss berechtigt ist diejenige Person, die zur Vertretung bei medizinischen Massnahmen befugt ist.

\subsection{Behördliche Massnahmen}

Behördliche Massnahmen des Erwachsenenschutzes haben das Wohl und die Interessen von schutz- und hilfsbedürftigen Personen sicherzustellen. Sie sind nur dann anzuordnen, wenn die Umgebung des Betroffenen nicht genügend Schutz bieten kann, z.B. durch Hilfeleistungen der Familie oder anderer nahestehender Personen. Behördliche Massnahmen müssen zwecktauglich sein, so wenig wie möglich in die Rechtsstellung der schutzbedürftigen Personen eingreifen und zumutbar sein. Dabei wird zwischen amtsgebundenen Massnahmen (Beistandschaften) und nicht amtsgebundenen Massnahmen (eigenes Handeln der Erwachsenenschutzbehörde und fürsorgerische Unterbringung) unterschieden. 
Das neue Erwachsenenschutzrecht kennt als einzige amtsgebundene Massnahme die Errichtung einer Beistandschaft. Der Beistand wird durch die Erwachsenenschutzbehörde ernannt und hat zur Aufgabe, das Wohl und den Schutz einer hilfsbedürftigen Person sicherzustellen (Art. 390ff ZGB). Eine Beistandschaft darf nur errichtet werden, wenn der Betroffene aufgrund eines im Gesetz umschriebenen Schwächezustandes (geistige Behinderung, psychische Störung o. ä.) oder wegen Urteilsunfähigkeit nicht mehr in der Lage ist, seine eigenen Angelegenheiten zu besorgen bzw. die erforderlichen Vollmachten zu erteilen. Die Beistandschaft wird auf Antrag der betroffenen Person oder einer nahestehenden Person oder von Amtes wegen errichtet.

Das Gesetz unterscheidet vier verschiedene Arten von Beistandschaften: (1) Begleitbeistandschaft, (2) Mitwirkungsbeistandschaft, (3) Vertretungsbeistandschaft und (4) umfassende Beistandschaft. Der Erwachsenenschutzbehörde obliegt es, die Beistandschaft möglichst massgeschneidert auf die Bedürfnisse der betroffenen Person auszurichten. Zum einen hat sie festzulegen, welche Art von Beistandschaft konkret errichtet werden soll. Zum anderen muss sie die Aufgabenbereiche des Beistandes möglichst genau umschreiben (Art. 391 ZGB). Die Aufgabenbereiche betreffen sowohl die Personensorge als auch die Vermögenssorge.

Die Beistandschaften können - mit Ausnahme der umfassenden Beistandschaft - miteinander kombiniert werden (Art. 397 ZGB).

Erscheint die Errichtung einer Beistandschaft wegen des Umfangs der Aufgaben als offensichtlich unverhältnismässig, so kann die Erwachsenenbehörde selbst für die betroffene Person handeln (Art. 392 ZGB). Sie kann insbesondere die Zustimmung zu einem Rechtsgeschäft erteilen oder einer Drittperson für einzelne Aufgaben einen Auftrag erteilen.

Im Rahmen einer fürsorgerischen Unterbringung kann eine Person gemäss Art. 426ff ZGB gegen oder ohne ihren Willen in eine geeignete Einrichtung eingewiesen werden (mehr dazu in Kap. 5.10.). Die fürsorgerische Unterbringung soll die betroffene Person in die Selbstständigkeit zurückführen und ihre Selbstverantwortung stärken.

Für die Anordnung der fürsorgerischen Unterbringung und die Entlassung ist grundsätzlich die Erwachsenenschutzbehörde zuständig (Art. 428 ZGB). Die Kantone können aber auch Ärztinnen und Ärzte bezeichnen, die befristet für eine Dauer von maximal sechs Wochen eine Person in einer Einrichtung unterbringen können (Art. 429 ZGB). Eine in einer Einrichtung untergebrachte Person hat das Recht, eine Vertrauensperson beizuziehen, die sie während des Aufenthalts und bis zum Abschluss aller damit zusammenhängenden Verfahren unterstützt (Art. 432 ZGB). Der Vertrauensperson kommt insbesondere die Aufgabe zu, die untergebrachte Person über ihre Rechte aufzuklären und ihr bei deren Wahrnehmung zu helfen. Des Weiteren wirkt sie bei der Erarbeitung des Behandlungsplans mit 
(Art. 433 ZGB). Die fürsorgerische Unterbringung schränkt die Bewegungsfreiheit der betroffenen Person ein, hat aber - anders als etwa die umfassende Beistandschaft - keine Auswirkungen auf deren Handlungsfähigkeit.

Wird eine Person zur medizinischen Behandlung einer psychischen Störung in eine psychiatrische Klinik eingewiesen, so hat der behandelnde Arzt gemäss Art. 433ff ZGB unter Beizug der betroffenen Person und gegebenenfalls ihrer Vertrauensperson zwingend einen schriftlichen Behandlungsplan zu erstellen. Dieser ist vor Beginn der Behandlung der betroffenen Person zur Zustimmung zu unterbreiten. Verweigert die betroffene Person die Zustimmung oder kann sie mangels Urteilsfähigkeit nicht zustimmen, so kann die Chefärztin oder der Chefarzt der Abteilung unter restriktiven Voraussetzungen die im Behandlungsplan vorgesehenen Massnahmen auch gegen den Willen des Betroffenen anordnen.

\subsection{Die Behörden des Erwachsenenschutzes}

Das neue Erwachsenenschutzrecht sieht zu seiner Umsetzung verschiedene Behörden vor. Zentrale Bedeutung kommt der Erwachsenenschutzbehörde und deren Aufsichtsbehörde zu. Daneben umschreibt das Erwachsenenschutzrecht bestimmte Funktionen, namentlich das Amt des Beistands. Ferner sind gewisse Aufgaben den Gerichten zugewiesen.

Die Erwachsenenschutzbehörde ist eine interdisziplinär zusammengesetzte Fachbehörde, die von den Kantonen eingesetzt wird (Art. 440 ZGB). Sie besteht aus mindestens drei Mitgliedern und entscheidet in der Regel als Kollegialbehörde. Die Erwachsenenschutzbehörde nimmt bei der Umsetzung des Erwachsenenschutzrechts eine Schlüsselstellung ein. Sie prüft, ob zum Schutz einer hilfsbedürftigen Person eine Beistandschaft errichtet werden muss, bestimmt die Art der Beistandschaft, ernennt einen Beistand und umschreibt dessen Aufgaben. Sie überwacht den Beistand und schreitet ein, wenn die Interessen der verbeiständeten Person gefährdet sind. Wichtige Aufgaben kommen der Erwachsenenschutzbehörde aber auch im Zusammenhang mit den nichtbehördlichen Massnahmen zu. So hat sie etwa den Vorsorgeauftrag auf seine Gültigkeit hin zu untersuchen, ihn auszulegen und nötigenfalls zu ergänzen, oder sie hat auf Antrag hin zu überprüfen, ob der Patientenverfügung entsprochen wird.

Die Aufsichtsbehörde überwacht die Tätigkeit der Erwachsenenschutzbehörde und sorgt für eine korrekte, einheitliche Rechtsanwendung (Art. 441 ZGB). Sie schreitet von Amtes wegen ein, wenn sie Kenntnis von fehlerhaftem Tun oder Unterlassen der Erwachsenenschutzbehörde erlangt. Im Rahmen der allgemeinen Aufsicht vermag die Aufsichtsbehörde Entscheide der Erwachsenenschutzbehörde im Einzelfall nicht zu korrigieren. Zur Neubeurteilung ist einzig das zuständige Gericht im Beschwerdeverfahren berechtigt. Die Kantone bestimmen die Aufsichtsbehörde und deren Organisation. 
Ist eine Person aufgrund eines Schwächezustandes hilfsbedürftig, so errichtet die Erwachsenenschutzbehörde eine Beistandschaft. Der Beistand übernimmt die ihm von der Erwachsenenschutzbehörde im Einzelfall zugewiesenen Aufgaben und gleicht die Hilfs- und Schutzbedürftigkeit der betroffenen Person aus. Das neue Erwachsenenschutzrecht kennt vier verschiedene Arten von Beistandschaften, die sich hinsichtlich der Intensität des Eingriffs in die Rechtsstellung der betroffenen Person stark unterscheiden. ${ }^{24}$ Der Begriff der Vormundschaft wird im neuen Erwachsenenschutzrecht nicht mehr verwendet.

Entscheide der Erwachsenenschutzbehörde können beim zuständigen kantonalen Gericht mit Beschwerde angefochten werden (Art. 450 ZGB).

\subsection{Verfahren}

Für die Anordnung und Aufhebung von behördlichen Massnahmen ist die Erwachsenenschutzbehörde am Wohnsitz der betroffenen Person zuständig (Art. 442 ZGB). Das Verfahren richtet sich grundsätzlich nach kantonalem Recht, wobei die Kantone gewisse bundesrechtliche Vorgaben zu beachten haben (Art. 443-450e ZGB). Die Erwachsenenschutzbehörde wird entweder von Amtes wegen oder auf private Meldung hin tätig (Art. 443 ZGB). Das heisst, jede Person kann der Erwachsenenschutzbehörde eine ihr hilfsbedürftig erscheinende Person melden. Bevor die Behörde eine Massnahme anordnet, muss sie die Hilfsbedürftigkeit der betroffenen Person umfassend abklären und hört diese an (Art. 444 ZGB).

In ihrer Funktion als Aufsichtsbehörde ist die Erwachsenenschutzbehörde Beschwerdeinstanz. So kann gegen jede Handlung oder Unterlassung des Beistandes die Erwachsenenschutzbehörde angerufen werden (Art. 419 ZGB).

Gegen Entscheide der Erwachsenenschutzbehörde können die betroffene Person oder Nahestehende Beschwerde beim zuständigen Gericht erheben. Das Gericht überprüft den Fall in rechtlicher und tatsächlicher Hinsicht umfassend und entscheidet neu (Art. 450a ZGB). Nach Ausschöpfung des kantonalen Rechtsweges ist die Beschwerde in Zivilsachen an das Bundesgericht möglich.

Gegen das (Nicht-)Handeln der Erwachsenenschutzbehörde kann ferner eine Beschwerde bei deren Aufsichtsbehörde erhoben werden. Im Gegensatz zur gerichtlichen Beschwerdeinstanz besitzt die Aufsichtsbehörde allerdings nicht die Kompetenz, einen Entscheid der Erwachsenenschutzbehörde umzustossen. Sie kann einzig die Erwachsenenschutzbehörde zur korrekten und einheitlichen Rechtsanwendung anhalten. 


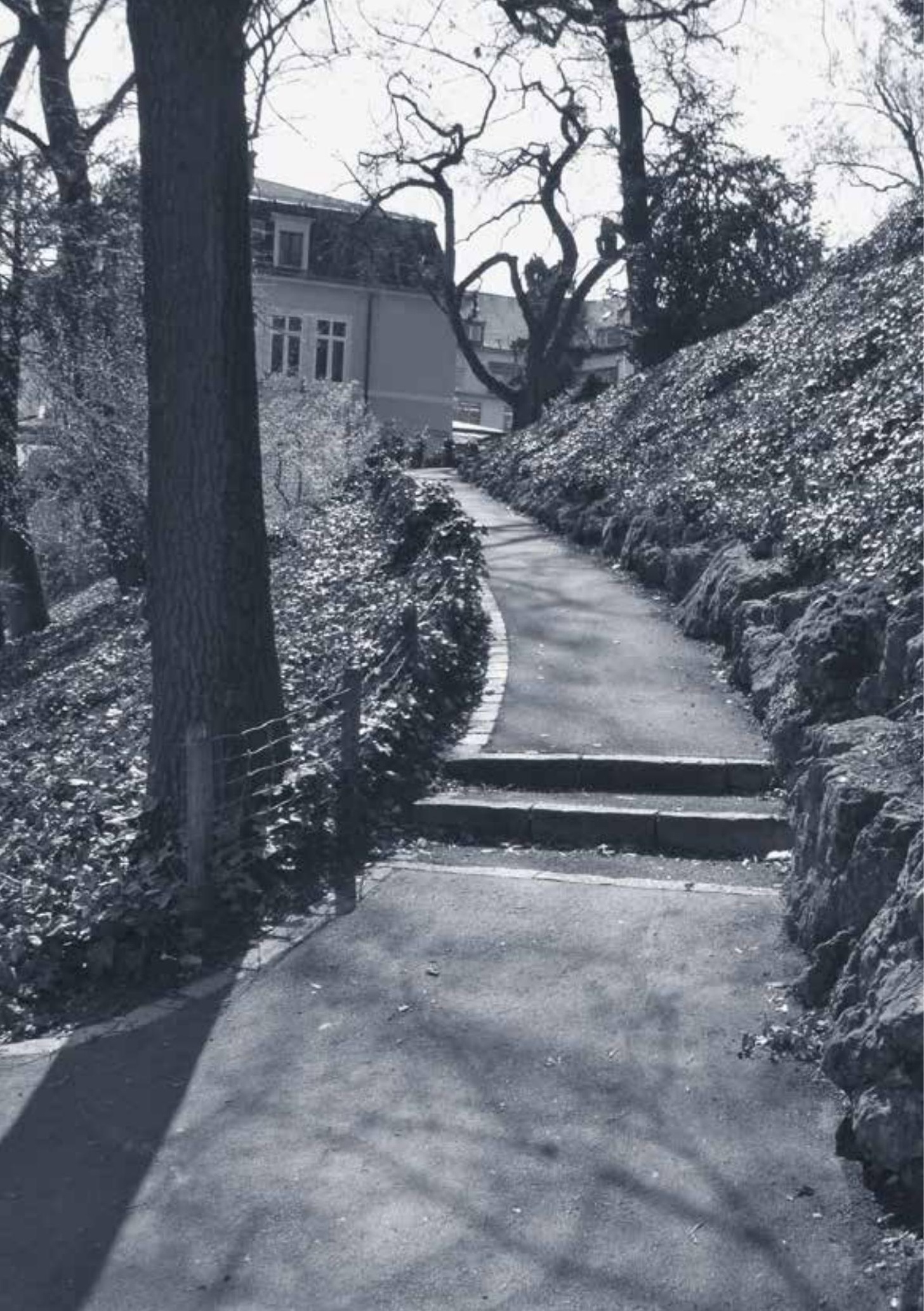




\title{
Grundlagen der Behandlung von Patientinnen und Patienten
}

\subsection{Behandlungsvertrag zwischen Arzt und Patient}

\author{
Die Art des Vertrags zwischen Ärztin und Patient beeinflusst die Ausgestaltung \\ des Arzt-Patienten-Verhältnisses. Es ist deshalb für den Arzt wichtig, sich im \\ Klaren darüber zu sein, wer mit wem einen Behandlungsvertrag abschliesst - \\ und dafür zu sorgen, dass dies auch für die Patientin klar ist.
}

Der Behandlungsvertrag kommt in der Regel bereits dadurch zustande, dass ein Patient einen Sprechstundentermin vereinbart. Damit der Behandlungsvertrag gültig ist, muss der Patient handlungsfähig sein, d.h., er ist sowohl volljährig als auch urteilsfähig.

Gemäss Zivilgesetzbuch können folgende Gründe dazu führen, dass eine Patientin oder ein Patient urteilsunfähig ist: Kindesalter, geistige Behinderung, psychische Störung, Rausch oder ähnliche Zustände, durch welche die Fähigkeit mangelt, vernunftgemäss zu handeln. ${ }^{25}$ Bei Kindern schliessen die Eltern als Inhaber der elterlichen Sorge den Behandlungsvertrag ab. ${ }^{26}$ Urteilsunfähige Volljährige werden grundsätzlich durch die Beiständin oder eine andere vertretungsberechtigte Person vertreten. ${ }^{27}$ Bei einer kurzfristigen vorübergehenden Urteilsunfähigkeit eines mündigen Patienten erfolgt die Behandlung - mangels eines gesetzlichen Vertreters - als Geschäftsführung ohne Auftrag.

Ist ein Patient urteilsfähig, aber minderjährig oder steht er unter umfassender Beistandschaft, kann er ohne die Zustimmung des gesetzlichen Vertreters «Rechte ausüben, die ihm um seiner Persönlichkeit willen zustehen». ${ }^{28}$ Der Abschluss eines Behandlungsvertrags ist ein höchstpersönliches Recht. Der urteilsfähige Minderjährige - beispielsweise eine Jugendliche - oder der Volljährige unter umfassender Beistandschaft kann deshalb nach der Lehrmeinung einiger Juristen ohne Zustimmung des gesetzlichen Vertreters einen Behandlungsvertrag abschliessen, wenn die Kosten durch eine Sozialversicherung gedeckt sind oder wenn es um eine alltägliche und nicht kostspielige Behandlung geht. Zentral ist hier die Frage der Urteilsfähigkeit des Patienten. Selbst wenn man aber davon ausgeht, dass in diesen Fällen kein Vertragsverhältnis begründet wird, dürfte das in den Fällen, in denen eine Sozialversicherung die Kosten übernimmt, keine Rolle 
spielen. Die Behandlung kann dennoch durchgeführt werden und der Arzt erhält sein Honorar. Grundsätzlich können fünf Grundformen von Behandlungsverträgen unterschieden werden, wobei es in der Praxis Mischformen gibt.

- die Behandlung in der Arztpraxis;

- die Behandlung im Belegarztspital;

- die Behandlung im Privatspital mit angestellten Ärzten;

- die Behandlung im öffentlichen Spital;

- die Behandlung im Pflegeheim.

Diese fünf Formen werden im Folgenden kurz skizziert.

\section{Die Behandlung in der Arztpraxis}

Das Verhältnis zwischen einem frei praktizierenden Arzt und seiner Patientin untersteht in der Regel dem Recht des einfachen Auftrages gemäss Obligationenrecht (OR). ${ }^{29}$ Dieser einfache Auftrag wird dadurch abgeschlossen, dass der Patient die Ärztin bittet, ihn zu untersuchen oder zu behandeln, und sie einen Termin vereinbaren. Eine Pflicht zur Übernahme des Auftrages bzw. eine Behandlungspflicht existiert für den Arzt nur in Notfällen oder wenn kantonales Recht dies ausnahmsweise vorschreibt. Als Pendant zur freien Arztwahl der Patienten gilt also auch grundsätzlich die freie Patientenwahl für den Arzt. ${ }^{30}$

Die Ärztin muss als Auftragnehmerin die ärztliche Handlung persönlich ausführen. Selbstverständlich heisst das nicht, dass sie in ihrer Praxis kein medizinisches Hilfspersonal beschäftigen darf. Sie trägt aber die Verantwortung dafür, dass dieses richtig instruiert, gemäss seinen Fähigkeiten eingesetzt und von ihr beaufsichtigt wird.

Der Arzt schuldet der Patientin keinen Behandlungserfolg. Hingegen ist er verpflichtet, seine Tätigkeit sorgfältig nach bestem Wissen und Gewissen und nach den Regeln der ärztlichen Kunst auszuführen (vgl. Kap. 9.3.). Dazu gehört insbesondere, dass die Behandlung medizinisch indiziert ist und dass der Arzt die notwendigen Kenntnisse und Fertigkeiten zu deren Ausführung besitzt. Die Ärztin ist dabei nicht einfach an die Weisungen und Wünsche der Patienten gebunden. Dem Willen des Patienten oder seiner Vertreter auf eine bestimmte Behandlung und Betreuung muss nur entsprochen werden, wenn diese Behandlung den allgemein anerkannten Regeln entspricht.

30 Art. 4 Abs. 3 und Art. 5 FMH-Standesordnung. Doch darf der Arzt Patienten nicht aus diskriminierenden Gründen ablehnen (Hautfarbe, Konfession usw.). 
Gegenstand des Auftrags sind Anamnese, Untersuchung, Diagnose, Beratung und Behandlung durch den Arzt. Die Ärztin muss den Patienten aufklären (vgl. Kap. 4.2). Nur so können sie gemeinsam das weitere Vorgehen, also den Behandlungsplan, festlegen. Nur wenn die Ärztin den Patienten korrekt aufgeklärt hat, kann dieser frei entscheiden, ob und welche Behandlung er wünscht.

Als Auftragnehmer ist der Arzt verpflichtet, der Patientin jederzeit Rechenschaft abzulegen (vgl. Kap. 9.1.). Er muss eine sachgerechte Krankengeschichte führen (vgl. Kap. 4.5.). Die Aufzeichnungs- oder Dokumentationspflicht ergibt sich auch aus der FMH-Standesordnung und aus den kantonalen Gesundheitsgesetzen. Die Standesordnung und teilweise auch die kantonalen Gesundheitsgesetze regeln zudem die Dauer der Aufbewahrungspflicht für die Krankengeschichten.

Der Behandlungsauftrag kann von beiden Seiten grundsätzlich jederzeit widerrufen werden. Für den Arzt gibt es hier aber Einschränkungen, wenn die Gesundheit der Patientin dadurch gefährdet oder beeinträchtigt wird. Bei einer Kündigung zur Unzeit kann der Kündigende schadenersatzpflichtig werden. ${ }^{31}$

\section{Die Behandlung im öffentlichen Spital}

Die öffentlichen Spitäler sind Anstalten mit einem Gemeinwesen als Träger. Das Verhältnis der angestellten Ärzte zum öffentlichen Spital richtet sich grundsätzlich nach den Vorschriften des kantonalen Rechts. Das Gleiche gilt daher für das Verhältnis zwischen dem Spital und den Patienten. Das Behandlungsverhältnis ist öffentlich-rechtlicher Natur und entweder als Anstaltsbenutzung oder als verwaltungsrechtlicher Vertrag ausgestaltet. Dabei enthält das kantonale Gesundheitsrecht in der Regel die Rahmenbedingungen, während die Details dann in Spitalreglementen und in den Verträgen festgehalten werden.

Auch hier ist ein gespaltenes Rechtsverhältnis mit zwei Verträgen denkbar: einer zwischen Chefärztin, Belegarzt oder Konsiliarärztin und Patient, und der andere zwischen Spital und Patient. Massgeblich ist die kantonale Spitalgesetzgebung: Entweder erlaubt sie die Chefarztsprechstunde als freiberufliche Tätigkeit oder der Chefarzt ist auch in seiner «Privatsprechstunde» Angestellter des Spitals.

Die ärztliche Tätigkeit an einem öffentlichen Spital gilt heute als amtliche Tätigkeit (gegebenenfalls mit Ausnahme der Chefarztsprechstunde). Die Haftung der Ärztinnen des öffentlichen Spitals richtet sich demnach primär nach dem kantonalen Verantwortlichkeits- bzw. Staatshaftungsrecht. Das Spital bzw. dessen Haftpflichtversicherer wird allfällige Schadenersatzverhandlungen mit dem Patienten führen müssen. Bei grobem Verschulden des Arztes wird das Spital aber in den meisten Kantonen intern auf den Arzt Rückgriff nehmen können - aber faktisch in aller Regel nicht tun.

31 Der selbstständig tätige Arzt hat sich zudem an die Berufspflichten gemäss Art. 40 MedBG zu halten. 
Die Dokumentations- und die Aufklärungspflicht der Ärztin sowie die Bedingung der Einwilligung des Patienten in die Behandlung ergeben sich aus dem kantonalen Spital- oder Gesundheitsrecht und dem FMH-Standesrecht. Während die kantonalen Vorschriften für Angestellte von öffentlichen Spitälern sowie für frei praktizierende Ärzte unterschiedliche Regeln enthalten können, gelten die Standesregeln gleichermassen für alle FMH-Mitglieder.

Die Aufnahme- oder Behandlungspflicht richtet sich nach dem kantonalen Gesundheitsrecht oder nach dem Leistungsauftrag zwischen Kanton und Spital. Die Pflicht geht hier in aller Regel über die blosse Nothilfepflicht hinaus. Da ein öffentliches Spital dem öffentlichen Recht untersteht, haben die Patienten zudem grundsätzlich Anspruch auf Gleichbehandlung. Kommt das Spital aber mit seiner Kapazität an eine Grenze, muss triagiert werden; dabei sollen medizinische Gründe ausschlaggebend sein.

\section{Die Behandlung im Belegarztspital}

In einem Belegarztspital gehen sowohl das Spital als auch die Belegärztin mit dem Patienten einen Vertrag ein: Das Belegarztspital tritt gegenüber dem Patienten nur für die nichtärztlichen Dienstleistungen als Vertragspartner auf; der Belegarzt schliesst seinerseits mit der Patientin einen Vertrag für die ärztliche Leistung ab. Man spricht deshalb von einem «gespaltenen Spitalvertrag». Durch die Abgrenzung der Leistungen zwischen Spital und Arzt beschränkt sich auch die Verantwortlichkeit der Beteiligten auf ihre jeweiligen Bereiche. Sinnvollerweise schliessen Arzt und Spital ihre Haftpflichtversicherungen beim selben Versicherer ab, damit es keine Schnittstellenprobleme gibt. Für die Belegärztin gilt bezüglich der Behandlungspflicht grundsätzlich dasselbe wie für den frei praktizierenden Arzt (vgl. oben). Vorbehalten bleibt aber ein allfälliger Vertrag des Belegspitals mit dem Kanton (Leistungsauftrag). ${ }^{32}$

\section{Die Behandlung im Privatspital mit angestellten Ärzten}

Schliesst auf Seiten des Leistungserbringers nicht der einzelne Arzt, sondern das Privatspital einen Vertrag mit dem Patienten für dessen Spitalaufenthalt ab, spricht man von einem «Gesamtbehandlungsvertrag». Die medizinische Behandlung untersteht wie beim frei praktizierenden Arzt dem Auftragsrecht. Für die übrigen Teile (Pflege, Unterkunft, Verpflegung usw.) muss im Einzelfall geprüft werden, welche Bestimmungen anwendbar sind. Da beim Gesamtbehandlungsvertrag das Spital als einziger Vertragspartner gegenüber dem Patienten auftritt, 
ist es in erster Linie auch für die medizinische Versorgung verantwortlich. Bei Behandlungsfehlern kann der Patient aber neben dem Spital als seinem Vertragspartner auch die fehlbare Ärztin wegen unerlaubter Handlung belangen; diesfalls muss allerdings das Spital seine Arbeitnehmer von Schadenersatzpflichten freihalten (vgl. Kap. 9.1.).

Bezüglich der Behandlungspflicht gilt dasselbe wie für den frei praktizierenden Arzt (vgl. oben). Vorbehalten bleibt auch hier aber neben dem kantonalen Recht ein allfälliger Vertrag des Spitals mit dem Kanton (Leistungsauftrag).

\section{Die Behandlung im Pflegeheim}

Bewohner von Pflegeeinrichtungen schliessen mit dem Heim einen Betreuungsvertrag ab, der Auskunft darüber gibt, welche Leistungen die Einrichtung im Bereich der Pflege und Betreuung erbringt. ${ }^{33}$ Ärztliche Leistungen werden vom Betreuungsvertrag nicht erfasst. Die ärztliche Grundversorgung der Bewohner erfolgt - je nach Entscheid des Patienten - entweder durch einen Heimarzt oder durch eine Belegärztin. Heimärzte können als Angestellte oder im Auftragsverhältnis tätig sein. Ihre Aufgaben ergeben sich aus dem Arbeitsvertrag oder einer Heimarztvereinbarung. Für die Belegärzte gelten die gleichen auftragsrechtlichen Regeln wie für frei praktizierende Ärzte.

In Wohn- und Pflegeeinrichtungen ist sowohl das Heimarzt- als auch das Belegarztmodell anzutreffen. Während im Spital die Arztwahl normalerweise eingeschränkt ist, ist im Pflegeheim ein reines Heimarztmodell unzulässig. Im Pflegeheim ist die freie Arztwahl als Aspekt der persönlichen Freiheit gewährleistet. ${ }^{34}$ Die Bewohner dürfen sich auch weiterhin durch ihre Hausärztin behandeln lassen. Dieser Unterschied zum Spital wird damit begründet, dass den persönlichen Bedürfnissen und Lebensgewohnheiten im Pflegeheim ein höherer Stellenwert zukommt, da der Aufenthalt regelmässig nicht nur von vorübergehender Natur ist. Die Einrichtungen dürfen vom Grundsatz der freien Arztwahl nur abweichen, wenn wichtige Gründe vorliegen. 


\section{Patientenpflichten im Rahmen einer Behandlung}

Der Behandlungsvertrag bzw. der Spitaleintritt schafft Rechte und Pflichten auf beiden Seiten, d.h. beim Leistungserbringer und bei der Patientin. Die Patientin oder an ihrer Stelle der Versicherer ist verpflichtet, eine korrekte Arzt- oder Spitalrechnung fristgerecht zu bezahlen. Der Patient ist angehalten, der Ärztin die notwendigen Auskünfte über sich und sein Leiden zu erteilen. ${ }^{35}$ Er muss zudem im Spital die Hausordnung respektieren, sich an die Weisungen des Personals halten und muss generell auf andere Patienten und das Personal Rücksicht nehmen.

\section{Verjährungsfristen}

Privatrechtliche Honorarforderungen verjähren binnen fünf Jahren. ${ }^{36}$ Rechnungen für Zusatzversicherungen muss der Patient aber innert zwei Jahren einreichen - also muss der Arzt hier vorher Rechnung stellen. Sonst riskiert er, seinen Honoraranspruch für die zusatzversicherten Behandlungen zu verlieren. Für die öffentlich-rechtlichen Forderungen ist die Verjährungsfrist im kantonalen Recht geregelt.

Haftpflichtansprüche gegen Ärzte und Privatspitäler aus dem Behandlungsvertrag verjähren mit Ablauf von zehn Jahren. ${ }^{37}$ Ausservertragliche Haftpflichtansprüche verjähren im Privatbereich ein Jahr ab dem Tag, an dem der Geschädigte Kenntnis vom Schaden und von der Person des Ersatzpflichtigen erlangt hat, jedenfalls aber nach zehn Jahren vom Tage der schädigenden Handlung an gerechnet. ${ }^{38}$ Im öffentlichen Bereich verjähren Haftpflichtansprüche meist nach kürzerer Frist (im kantonalen Recht geregelt, einige Kantone kennen ein kurze Verwirkungsfrist nach Kenntnis des Fehlers). (Siehe zur Haftpflicht auch Kap. 9.2.)

Obliegenheiten sind Pflichten, die nicht direkt rechtlich eingeklagt werden können. Ein Patient, der eine Obliegenheit verletzt, muss deswegen keinen Schadenersatz bezahlen, kann aber seinen Schadenersatzanspruch gegenüber dem Arzt teilweise oder vollständig verlieren, wenn er ihm eine wichtige Information verschwieg.

36 Art. 128 Ziff. 3 OR.

37 Art. 127 OR.

38 Art. 127 OR. 


\subsection{Aufklärung des Patienten}

\section{Damit ein Patient rechtsgültig in eine Behandlung einwilligen kann, muss er ausreichend aufgeklärt sein. Der Arzt oder das Spital müssen im Streitfall die genügende Aufklärung nachweisen können.}

Eine angemessene Patientenaufklärung ist für jede Behandlung wichtig. Sie ist eine Bedingung, um den Behandlungsvertrag zu erfüllen, und stärkt darüber hinaus das Vertrauen zwischen Arzt und Patientin.

Bei Operationen und anderen invasiven Behandlungen - Schneiden, Stechen, Bestrahlen, Verabreichen von Arzneimitteln - ist die korrekte Patientenaufklärung und Einwilligung zudem aus strafrechtlicher Sicht zentral: Sie ist der strafrechtliche Rechtfertigungsgrund für den Eingriff, der gemäss Bundesgerichtspraxis den objektiven Tatbestand der Körperverletzung und/oder Tötung erfüllt. Liegt ein Rechtfertigungsgrund vor, macht sich die Ärztin nicht strafbar. Auch im Persönlichkeits- und Grundrechtsschutz gilt die Einwilligung als Rechtfertigungsgrund. Weil der Eingriff dann erlaubt ist, entfallen Schadenersatz- und/oder Genugtuungszahlungen, sofern die Ärztin sorgfältig behandelt.

Die Operateurin und der Anästhesist dürfen zwar die Aufklärung delegieren, beispielsweise an den Assistenzarzt oder die zuweisende Ärztin. Weil sie aber den Eingriff durchführen, tragen sie die Konsequenzen, wenn die delegierte Aufklärung ungenügend war. In einem solchen Falle wäre die Zustimmung des Patienten zum Eingriff rechtlich nicht gültig und der invasive Eingriff damit widerrechtlich.

Es gibt in der Schweiz keine fixe Prozentgrenze, oberhalb derer Risiken mitgeteilt werden müssen.$^{39}$ Die Standardformel des Bundesgerichts zur Aufklärung lautet: «Der Patient soll über den Eingriff oder die Behandlung soweit unterrichtet sein, dass er seine Einwilligung in Kenntnis der Sachlage geben kann. Die Aufklärung darf jedoch keinen für seinen Gesundheitszustand schädlichen Angstzustand hervorrufen. $»^{40}$ Daraus ergibt sich ein eigentlicher Optimierungsauftrag an die Medizin: Damit die Patienten die Information auch verstehen und «verdauen» können, ist es bei medizinisch nötigen Eingriffen nach Meinung des FMH-Rechtsdienstes angezeigt, etwa die fünf häufigsten oder schwersten Risiken zu erläutern; dies sollte ohne besondere Nachfrage des Patienten erfolgen. Allerdings lässt sich aus der schweizerischen Rechtsprechung, welche die Anforderungen an eine genügende Aufklärung festlegt, nicht allgemein ableiten, über welche Risiken aufgeklärt werden muss. Die Urteile betreffen Einzelfälle und bis anhin können daraus keine Rezepte für die Risikoaufklärung abgeleitet werden. Das bedeutet für den Arzt, dass er bei jedem Patienten abschätzen muss, welche Risiken sich bei 
ihrer Verwirklichung so schwer auf dessen Leben auswirken würden, dass eine Aufklärung gerechtfertigt ist. Unterlässt der Arzt die Aufklärung über ein Risiko und verwirklicht sich dieses dann, kann er sich haft- und strafbar machen, selbst wenn die Behandlung an sich sorgfältig war. Ist ein Eingriff hingegen medizinisch nicht indiziert, etwa weil der Eingriff allein aus ästhetischen Gründen erfolgt, muss der Arzt möglichst über alle Risiken aufklären.

Wenn die Patientin zu erkennen gibt, dass sie mehr oder weniger erfahren möchte, kann und soll dieser Wunsch respektiert werden; ein Verzicht der Patientin auf die Aufklärung im üblichen Umfang soll in der Krankengeschichte notiert werden.

Neben diesen juristischen Aspekten hat die Aufklärung auch für die Medizin selbst eine grosse Bedeutung. Sie dient der Vertrauensbildung und dem Sicherheitsgefühl der Patientin. Eine gute Aufklärung dürfte damit auch die Erfolgschancen einer Behandlung erhöhen.

\section{Inhalt der Aufklärung}

Die Konzentrations- und Aufnahmefähigkeit der Patienten ist krankheitsbedingt oft eingeschränkt. Der Arzt muss den «Patienten in klaren, verständlichen Worten und so vollständig wie möglich» über die Behandlung aufklären. ${ }^{41}$ Die Patientin muss über alle Umstände informiert werden, «die im Hinblick auf die vorgesehenen medizinischen Massnahmen wesentlich sind, insbesondere über deren Gründe, Zweck, Art, Modalitäten, Risiken, Nebenwirkungen und Kosten, über Folgen eines Unterlassens der Behandlung sowie über allfällige alternative Behandlungsmöglichkeiten». ${ }^{42}$ Falls es sich um eine nichtetablierte Behandlung handelt, muss auch darüber informiert werden. Der Arzt hat seinen Patienten auch über Behandlungsalternativen aufzuklären, falls solche existieren. Gibt es Alternativen, muss der Patient entscheiden, welche Behandlung durchgeführt wird. Die Wahl der Operationsmethode liegt aber im Ermessen des Arztes.

Das Bundesgericht hat 1999 wie folgt präzisiert: «Die Aufklärung ist ein Austausch zwischen Arzt und Patient. Sie verlangt nach einer beidseitigen Mitwirkung. Ein Patient, der aufgrund seines Vorwissens gegen eine bestimmte Behandlungsart Bedenken hat, kann sich über Alternativen näher erkundigen. Tut er dies nicht oder nicht mit dem nötigen Nachdruck, so trifft ihn für sein Aufklärungsdefizit zumindest eine Mitverantwortung.» ${ }^{43}$

Die Patientin muss auch die nötigen Verhaltensanweisungen erhalten. Darf sie noch Auto fahren? Muss sie unbedingt auf die Einnahme von weiteren nicht ärztlich verordneten Medikamenten auf eigene Initiative verzichten? So verpflichtete

41 BGE 119 || 456

42 BGE 124 IV 258 E. 2; BGE 117 lb 197 E. 2a; Art. 7 und Art. 10 Abs. 2 BV; Art. 28 ZGB; Art. 377 Abs. 2 ZGB; Art. 398 OR; Art. 122 ff. StGB; Art. 5 Übereinkommen über Menschenrechte und Biomedizin; Art. 10 Standesordnung.

43 Nicht veröffentlichtes Urteil des Bundesgerichtes vom 8. April 1999. 
das Bundesgericht im Jahr 2001 in einem umstrittenen Entscheid das Waadtländer Universitätsspital CHUV zur Bezahlung der Hälfte des Schadens, weil das Gericht nicht überzeugt war, dass eine HIV-Patientin bei einer Tri-Therapie deutlich genug vor einer Selbstmedikation gewarnt worden war.

Der Patient muss auch über die Kosten informiert sein, vor allem, wenn er die Behandlungskosten ganz oder teilweise selbst übernehmen muss. Dies betrifft insbesondere die Behandlung auf der Privat- oder Halbprivatabteilung oder die Verschreibung von Originalmedikamenten, wenn ein Generikum auch geeignet wäre (vgl. Kap. 4.14.). Behandlungen auf Kosten der obligatorischen Krankenpflegeversicherung müssen wirksam, zweckmässig und wirtschaftlich sein. Wenn Anzeichen dafür bestehen, dass der Patient eine wirksame, aber nicht kassenpflichtige Behandlung selbst bezahlen will, soll der Arzt ihn über diese Option informieren.

\section{Eingriffsspezifische Aufklärungsprotokolle}

Weil nicht immer klar ist, ob ein Patient den Inhalt eines Aufklärungsgesprächs versteht, ist für die Operationsaufklärung und für andere komplexere Behandlungen ein Aufklärungsprotokoll mit Skizze zu empfehlen. Dieses wird im Gespräch mit dem Patienten ausgefüllt. Der Patient erhält nach dem Gespräch eine Kopie des Protokolls. Studien zeigen, dass schriftlich aufgeklärte Patienten den Eingriff besser verstehen und sich auch nach der Operation besser an die Information erinnern als bei bloss mündlicher Aufklärung. Die Patienten können die schriftliche Information mehrmals lesen, Angehörigen und Freunden vorlegen und mit ihnen den bevorstehenden Eingriff diskutieren. Aus Sicht der FMH sind operationsspezifische Aufklärungsprotokolle, die bereits einen sorgfältig formulierten Informationstext zur konkreten Operation enthalten, die besten Hilfsmittel für die Patientenaufklärung und Aufklärungsdokumentation.

\section{Zeitpunkt der Aufklärung}

Zum Aufklärungszeitpunkt gibt es noch keine klare Rechtsprechung des Bundesgerichts. Jedenfalls muss den Patienten bei schweren Operationen ohne zeitliche Dringlichkeit eine vernünftige Bedenkzeit eingräumt werden. Gemäss der Guideline Aufklärung der FMCH richtet sich der Aufklärungszeitpunkt nach der Dringlichkeit des Eingriffs und der Grösse der zu erwartenden Risiken. Je dringlicher der Eingriff ist, desto kurzfristiger kann die Aufklärung erfolgen. Je grösser die Risiken sind, desto früher muss aufgeklärt werden. Bei der Umsetzung dieses Grundsatzes haben sich folgende Faustregeln etabliert: Bei stationären risikoarmen Operationen (Routineeingriffen) ist bei der Planung des Eingriffes aufzuklären, spätestens am Nachmittag des Vortages. Bei risikoreichen Eingriffen hat die Aufklärung mindestens drei Tage vor der Operation zu erfolgen. ${ }^{44}$ 


\section{Beweislast und Dokumentation}

Das Spital bzw. die den Eingriff durchführende Ärztin tragen die Beweislast für die korrekte Aufklärung über die Risiken von Operationen und anderen invasiven Eingriffen. Deshalb ist die Dokumentation zentral: Im Streitfall muss auch Jahre später noch vernünftig rekonstruierbar sein, über was der Patient informiert worden ist. Kann die genügende Risikoaufklärung nicht bewiesen werden, haften Spital und/oder Ärztin für die Komplikationen des Eingriffs auch dann, wenn kein Behandlungsfehler vorliegt.

\subsection{Einwilligung}

Der Wille eines Patienten muss respektiert werden. Dies ist Voraussetzung für jede medizinische Behandlung und Betreuung. Gegen den erklärten Willen einer urteilsfähigen Patientin zu handeln, ist - von seltenen Ausnahmen abgesehen - rechtswidrig. Dies gilt auch dann, wenn der Wille der Patientin ihren eigenen Interessen zuwiderzulaufen scheint. Allerdings muss dem Wunsch nach einer bestimmten Behandlung und Betreuung nur entsprochen werden, wenn diese medizinisch indiziert sind.

Von einer informierten Einwilligung (informed consent) spricht man, wenn folgende Voraussetzungen erfüllt sind:

- Der Patient ist urteilsfähig.

- Er ist in ausreichendem Mass aufgeklärt (vgl. Kap.4.2.).

- Er ist in der Lage, die Reichweite seiner Einwilligung zu verstehen.

- Er kann seine Wahl unbeeinflusst von Zwang oder Manipulation äussern.

- Die Urteilsfähigkeit muss in der konkreten Situation im Hinblick auf eine bestimmte Handlung (Einwilligung) vorliegen. In der Regel kann die Ärztin bei einem volljährigen und wachen Patienten von dessen Urteilsfähigkeit ausgehen. Bestehen Zweifel, können nachfolgende Kriterien hilfreich sein:

- Ist die Patientin fähig, die Informationen in Bezug auf die zu fällende Entscheidung zu verstehen?

- Ist sie fähig, die Situation und die Konsequenzen, die sich aus alternativen Möglichkeiten ergeben, richtig abzuwägen?

- Ist sie in der Lage, die erhaltene Information im Kontext eines kohärenten Wertsystems rational zu gewichten?

- Kann sie die eigene Wahl äussern?

Auch Minderjährige oder Personen unter umfassender Beistandschaft können bezüglich einer Einwilligung in eine Behandlung urteilsfähig sein. Bei Patienten in der Altersstufe von 12 bis 16 Jahren kommt es auf das jeweilige Kind und die Art des Eingriffs an; die Urteilsfähigkeit muss von Fall zu Fall abgeklärt werden. Demgegenüber darf bei Jugendlichen ab 16 Jahren von deren Urteilsfähigkeit 
ausgegangen werden, wenn es nicht um einen aussergewöhnlichen Eingriff mit erheblicher Tragweite geht. Die Urteilsfähigkeit wird fallbezogen beurteilt. Im konkreten Fall muss geprüft werden, ob die jugendliche Patientin aufgrund ihrer geistigen Reife in der Lage ist, die Tragweite der Entscheidung richtig einzuschätzen, ihren Willen äussern und entsprechend handeln kann. Trifft dies zu, ist sie allein zuständig für die Erteilung der Einwilligung zu einem Eingriff. In der Rechtslehre wird die Auffassung vertreten, dass sie auch den Behandlungsvertrag ohne Zustimmung des gesetzlichen Vertreters abschliessen kann, wenn es um eine alltägliche und nicht kostspielige Behandlung geht, oder wenn die Kosten durch eine Krankenversicherung gedeckt sind (vgl. Kap. 4.1.). Zur Vermeidung von Haftungsrisiken ist zu empfehlen, grundsätzlich sowohl den vermutlich urteilsfähigen Jugendlichen als auch zusätzlich dessen gesetzliche Vertreter in die Aufklärung einzubeziehen und von beiden die Einwilligung einzuholen; dies gilt umso mehr bei kostspieligen Behandlungen oder bei Eingriffen, die den Rahmen gewöhnlicher und notwendiger Behandlungen übersteigen (z.B. bei Schönheitsoperationen). ${ }^{45}$

\section{Stellvertretende Einwilligung bei fehlender Urteilsfähigkeit}

Bei urteilsunfähigen Kindern liegt die Berechtigung zur stellvertretenden Einwilligung bei den Eltern als Inhabern der elterlichen Sorge oder bei einem Beistand. Gemeinsam sorgeberechtigte Eltern haben sich über die Zustimmung zu verständigen, wobei sie den andern zum Entscheid ermächtigen können. Der Entscheid der gesetzlichen Vertreter muss sich in jedem Fall am Kindeswohl orientieren.

Bei urteilsunfähigen Erwachsenen ist vorerst zu prüfen, ob sie sich nicht in einer Patientenverfügung zur medizinischen Behandlung geäussert haben. Die Anordnungen einer rechtsgültig errichteten Patientenverfügung sind grundsätzlich verbindlich (vgl. dazu unten). Fehlt eine Patientenverfügung, so hat die Ärztin unter Beizug der zur Vertretung bei medizinischen Massnahmen berechtigten Person die erforderliche Behandlung zu planen. Das Gesetz hat die Reihenfolge der Vertretungsberechtigung im Sinne einer klaren siebenstufigen Hierarchie festgelegt (mehr dazu siehe Kap. 3.). Reicht in dringlichen Fällen die Zeit nicht aus, um den Entscheid der vertretungsberechtigten Person einzuholen, so ergreift die Ärztin oder der Arzt medizinische Massnahmen nach dem mutmasslichen Willen und den Interessen der urteilsunfähigen Person. ${ }^{46}$ 
Form

Grundsätzlich sind Aufklärung und Einwilligung an keine Formvorschriften gebunden. Sie können also auch mündlich durchgeführt werden. Da das Beweisrisiko des Arztes hoch ist und sich die Patientin besser orientieren kann, wenn sie (auch) durch ein Aufklärungsformular geführt wird und dieses nochmals nachlesen kann, empfiehlt sich die schriftliche oder zumindest schriftlich festgehaltene Aufklärung mit Unterschrift der Patientin. Bei Forschungsvorhaben und in der Reproduktionsmedizin gilt die Formvorschrift der Schriftlichkeit.

\subsection{Patientenverfügung}

Die rechtliche Tragweite der Patientenverfügung ist neu im Zivilgesetzbuch geregelt. ${ }^{47}$ Die vom Verfasser des Dokumentes festgehaltenen Anordnungen haben die Wirkung einer verbindlichen Willensäusserung des Patienten. ${ }^{48}$ Der früher teilweise vertretene Standpunkt, dass die Patientenverfügung nur ein Indiz bei der Ermittlung des mutmasslichen Willens des Patienten sei, ist damit überholt. Der Entscheid über eine medizinische Behandlung ist ein sogenanntes höchstpersönliches Recht. Deshalb ist jede urteilsfähige Person berechtigt, eine Patientenverfügung zu errichten. Insbesondere steht dieses Recht auch urteilsfähigen Minderjährigen und Personen unter umfassender Beistandschaft zu.

Die Patientenverfügung bedarf der Schriftform. Eine eigenhändige Niederschrift ist nicht erforderlich, sondern es können auch standardisierte Formulare oder Textbausteine verwendet werden. ${ }^{49}$ Das Dokument ist nur gültig, wenn es datiert und vom Verfasser eigenhändig unterzeichnet ist. Die Patientenverfügung kann auch in einen Vorsorgeauftrag (mehr dazu siehe Kap. 3.) integriert werden. Es ist Sache des Verfassers, die Patientenverfügung den möglichen Adressaten zur Kenntnis zu bringen. Der Gesetzgeber hatte vorgesehen, dass der Patient auf der Versichertenkarte auf die Existenz der Patientenverfügung und den Hinterlegungsort aufmerksam machen kann; nachdem die Versichertenkarten erster Generation nicht einen einheitlichen technischen Standard einhalten, fehlt es zurzeit an der Kompatibilität mit den Health Professional Cards (HPC).

Der Inhalt der Patientenverfügung besteht in der Zustimmung oder Nichtzustimmung zu einer bestimmten medizinischen Massnahme für den Fall der Urteilsunfähigkeit des Verfassers. Zudem kann darin eine Vertrauensperson bezeichnet werden, die die Behandlung mit der Ärztin oder dem Arzt bespricht und in ihrem Namen entscheiden soll.

48 Bei fürsorgerischen Unterbringungen ist eine Patientenverfügung "zu berücksichtigen» (mehr dazu Kap. 4.10.). 
Die Feststellung der Wirksamkeit der Patientenverfügung ist Sache des Arztes. Er stellt insbesondere fest, ob der Patient urteilsunfähig ist und die vom Patienten in Aussicht genommene Situation eingetreten ist. Bei der Zustimmung mittels einer Patientenverfügung trifft den Arzt keine Aufklärungspflicht. Es darf ohne Weiteres davon ausgegangen werden, dass der Verfasser über die für die Willensbildung erforderlichen Informationen verfügte und auf zusätzliche Aufklärung verzichtet. Ist der Wille der betroffenen Person hinreichend klar, so ist er verbindlich und gilt als ihr wirklicher Wille im Zeitpunkt des Eingriffs. Der Arzt entspricht der Patientenverfügung, «ausser wenn diese gegen gesetzliche Vorschriften verstösst oder wenn begründete Zweifel bestehen, dass sie auf freiem Willen beruht oder noch dem mutmasslichen Willen des Patienten entspricht» ${ }^{50}$ Rechtlich unzulässig wäre auch die Anordnung eines nicht indizierten Eingriffes oder einer risikoreichen Massnahme, wenn ein weniger riskanter Eingriff mit identischem therapeutischem Nutzen zur Verfügung steht.

FMH und SAMW haben 2011 gemeinsam Vorlagen für eine Patientenverfügung ausgearbeitet (Kurzversion und ausführliche Version), die auf den Websites von FMH und SAMW unentgeltlich heruntergeladen werden können. ${ }^{51}$

\subsection{Führung der Krankengeschichte}

\section{Das Auftragsrecht, die FMH-Standesordnung und viele kantonalen Gesundheits- gesetze verpflichten die Ärztin und den Arzt, eine sachgerechte Krankenge- schichte zu führen. Diese muss die relevanten Untersuchungen, Behandlungen und die Überlegungen dazu festhalten.}

Die Gesetze regeln nicht im Detail, wie eine Krankengeschichte (KG) richtig geführt werden muss; ${ }^{52}$ dies wäre auch nicht sinnvoll, weil sich die KG einer Orthopädin und jene eines Psychiaters unterscheiden. Die korrekte KG-Führung ist damit primär eine medizinische Fachfrage.

Bei der Führung der KG geht es um zwei Ziele: die Patientensicherheit und die Transparenz. Um die Sicherheit zu gewährleisten, muss die KG lesbar sein. Sowohl der behandelnde Arzt wie auch eine allfällige Stellvertreterin oder Nachfolgerin müssen die Behandlung sicher fortsetzen können. Die Transparenz ist dann gegeben, wenn die KG alle wichtigen Befunde und Überlegungen enthält. Damit kann gegebenenfalls bei einer Behandlungsfehlerdiskussion die Behandlungsgeschichte

50 Art. 372 Abs. 2 ZGB.

51 Lucia Rabia, Michelle Salathé, Jacqueline Wettstein, Wissen was der Patient will, Schweizerische Ärztezeitung 2011; 92: 23/24 875; www.fmh.ch/service/patientenverfuegung.html; www.samw.ch/de/Ethik/Patientenverfuegung.html

52 Sonderregeln für die KG-Führung gibt es in einigen neueren Spezialgesetzen des Bundes (vgl. Kap. 5.1. bis 5.5.). 
nachvollzogen werden. Zur Transparenz gehört auch, dass der Arzt Korrekturen in der KG datiert und mit Initialen signiert. Denn nachträglich muss erkennbar sein, wann ein falscher Befund oder eine falsche Diagnose geändert wurden.

Eine unbrauchbare KG oder ein unkorrekter Operationsbericht können sich negativ auf eine Diskussion um Behandlungsfehler auswirken und zu einer Beweiserleichterung für den Patienten führen.

Die ärztliche Berufsausübung steht schliesslich unter der Aufsicht der Gesundheitsdirektion des Kantons; die KG muss deshalb nötigenfalls auch dem Kantonsarzt oder einem von ihm beauftragten ärztlichen Gutachter ermöglichen, die Behandlungen kritisch nachzuvollziehen.

\section{Elektronische KG-Führung}

Die elektronische KG-Führung ist erlaubt; dabei muss die elektronische Dokumentation sicherstellen, dass jederzeit nachvollziehbar ist, wer wann welche Eintragungen gemacht hat (Logging-Funktionen; kein Gruppen-Account). Das gilt auch für Änderungen. Bei Befunden und Berichten ist es daher wichtig, mit Versionen zu arbeiten, damit nachvollziehbar bleibt, welche Version zu welchem Zeitpunkt Gültigkeit hatte (vorläufiger Bericht, definitiver Bericht). Auch bei der Verlaufsdokumentation ist darauf zu achten, dass neue Eintragungen nicht alte überschreiben. Die Rekonstruierbarkeit des Dokumentationsstandes zu einem bestimmten Zeitpunkt ist wichtig, denn sonst hätte die KG in einer Behandlungsfehlerdiskussion wenig Beweiswert.

\section{Sicherheit und Aufbewahrungsfristen}

Egal, ob die KG auf Papier oder elektronisch geführt wird, der Arzt und die Ärztin müssen dafür sorgen, dass sie sicher ist. Dies hat verschiedene Konsequenzen:

Auch das Personal der Arztpraxis und der Spitäler untersteht dem Patientengeheimnis. Die Ärztin soll ihre Hilfspersonen schriftlich darauf verpflichten. ${ }^{53}$ Eine solche Verpflichtung ist im Mustervertrag für MPA eingebaut - aber er ist auch für das Reinigungspersonal und den Buchhalter sinnvoll. Für Personen, welche die Arztpraxis im IT-Bereich unterstützen, ist eine solche Verpflichtung unbedingt zu empfehlen. Kürzlich bekanntgeworden sind Fälle aus Deutschland, bei denen IT-Support leistende Personen die Krankengeschichten der betreuten Arztpraxen «abgesaugt» (kopiert) und an die pharmazeutische Industrie verkauft haben. 
Die KGs gehören in abschliessbare feuerfeste Schränke sowie an einen Ort, an dem sie bei einer Überschwemmung nicht beschädigt werden können.

Die KG muss in der Arztpraxis mindestens zehn Jahre nach der letzten Eintragung aufbewahrt werden. Für die öffentlichen Spitäler sehen einige Kantone längere Fristen vor. Immer dort, wo es um langfristige Leistungen der Versicherer gehen kann, kann es für den Patienten hilfreich sein, wenn der Arzt die KG auch ohne gesetzliche Pflicht länger aufbewahrt. Konkret ist zu denken an:

- Symptome oder Leiden, die später als Berufsunfall oder Berufskrankheit gemäss UVG relevant werden können; ${ }^{54}$

- Symptome oder Leiden, die einen Zusammenhang mit dem Militärdienst haben können.

Für elektronische Dokumentationen gelten analoge Regeln: Es müssen kontinuierlich oder wenigstens regelmässig Sicherheitskopien erstellt werden, die an einem anderen Ort oder wenigstens an einem Ort, an dem sie vor Wasser und Feuer geschützt sind, aufzubewahren sind. Auch die Sicherheitskopien sind entsprechend vor unbefugtem Zugang zu schützen.

Bei portablen Medien (Notebooks, Tablet-PCs) sollte die Abspeicherung der Daten verschlüsselt erfolgen, damit die Daten bei Verlust/Diebstahl nicht lesbar sind.

Bei Nutzung von Speichern im Internet («Cloud») ist darauf zu achten, dass die Daten vor dem Speichern in der Cloud lokal verschlüsselt werden. Bei der Auswahl des Anbieters sollen seine Datensicherheits- und Datenschutzregeln kritisch geprüft und sein diesbezüglicher Ruf beachtet werden; die verbreitetste Lösung ist nicht immer die beste.

\subsection{Arzneimittel, Betäubungsmittel und Medizinprodukte}

Das Heilmittelgesetz (HMG) will sicherstellen, «dass nur qualitativ hoch stehende, sichere und wirksame Heilmittel in Verkehr gebracht werden». Der Bund soll zudem darauf achten, dass «für die Forschung und Entwicklung im Heilmittelbereich günstige Rahmenbedingungen bestehen». ${ }^{55}$ Die zweite Zweckbestimmung kann die Behörde, die den Preis festlegen muss, in einen Zielkonflikt bringen, verlangt doch das KVG eine wirtschaftliche Versorgung.

54 Auch für Spätfolgen eines Berufsunfalls bleibt die Unfallversicherung des damaligen Arbeitgebers zuständig. Dies gilt für alle UVG-Leistungen, d.h. für Nachbehandlungen wie für Taggeld- und Rentenleistungen. Bei Berufskrankheiten ist die zeitliche Zuordnung ebenfalls wichtig, siehe die bekannte Asbestthematik: Heute wäre man froh, man hätte noch möglichst alle medizinischen Unterlagen zur Verfügung. Internationaler Konsens ist deshalb für arbeitsmedizinischtätige Ärztinnen und Ärzte eine Aufbewahrungsfrist von 40 Jahren. Siehe Anhang 4 zur FMH-Standesordnung.

55 Art. 1 Abs. 1 HMG. Unter dem Begriff Heilmittel versteht das HMG sowohl Arzneimittel als auch Medizinprodukte. 


\section{Arzneimittel}

Arzneimittel sind gemäss HMG: «Produkte chemischen oder biologischen Ursprungs, die zur medizinischen Einwirkung auf den menschlichen oder tierischen Organismus bestimmt sind oder angepriesen werden, insbesondere zur Erkennung, Verhütung oder Behandlung von Krankheiten, Verletzungen und Behinderungen; zu den Arzneimitteln gehören auch Blut und Blutprodukte.» ${ }^{56}$ Das Schweizerische Heilmittelinstitut Swissmedic lässt Arzneimittel zu, wenn diese sicher und wirksam sind. Die von den Pharmaunternehmen angegebenen Indikationen und die Dosis werden überprüft. Swissmedic kontrolliert zudem die verschiedenen Aspekte der Informationen, die zuhanden der Gesundheitsfachpersonen veröffentlicht werden, vor allem im Arzneimittelkompendium, sowie die Patienteninformationen in den Arzneimittelpackungen.

Das HMG legt Regeln für die Arzneimittel fest, die entsprechend den sogenannten "Magistralrezepturen» hergestellt werden. ${ }^{57}$ Dabei handelt es sich um Produkte, die der Apotheker auf der Grundlage einer individuellen ärztlichen Verordnung für einen bestimmten Patienten herstellt. Im Gegensatz zu den anderen Arzneimitteln werden Magistralrezepturen nicht in Serie hergestellt, sondern entsprechen einem spezifischen Bedarf; sie dürfen nicht an Lager gehalten werden. Solche Arzneimittel können insbesondere dann von Nutzen sein, wenn kein analoges, von Swissmedic zugelassenes Produkt auf dem Markt erhältlich ist.

Wenn Swissmedic ein Arzneimittel zulässt, ist noch nicht gewährleistet, dass die Kosten für dieses Arzneimittel durch die Krankenversicherer rückvergütet werden. Über diese Kassenpflichtigkeit entscheidet das Bundesamt für Gesundheit (BAG), nachdem es die Eidgenössische Arzneimittelkommission (EAK) angehört hat. Während Swissmedic also die Sicherheit und Wirksamkeit von Arzneimitteln überprüft, beurteilt das BAG deren Kosten-Nutzen-Verhältnis (vgl. Kap. 4.14.).

Die Verordnung von Arzneimitteln - also die Anwendung am Patienten, die Abgabe $^{58}$ und die Verschreibung - muss nach den «anerkannten Regeln der medizinischen und pharmazeutischen Wissenschaften ${ }^{59}$ erfolgen. Zudem muss der verordnende Arzt den Gesundheitszustand des Patienten kennen. ${ }^{60}$ Jeder Arzt, der über eine Praxisbewilligung verfügt, die von der zuständigen Behörde des Kantons erteilt wurde, in dem er seine Tätigkeit ausübt, ist gleichzeitig auch berechtigt, Arzneimittel zu verordnen. Assistenzärztinnen und Assistenzärzte dürfen auf den Rezeptformularen ihres Arbeitgebers (Spital oder frei praktizierender Arzt) ebenfalls Arzneimittel verordnen, wobei dies unter der Aufsicht und Verantwor-

58 Abgabe durch den Arzt mit Selbstdispensationsbewilligung (SD) nach kantonalem Gesundheitsrecht oder durch den Arzt ohne SD bei Notfallbehandlung oder je nach Kanton auch als Erstabgabe bei Behandlungsaufnahme. 
tung der Ärztinnen und Ärzte erfolgt, denen sie unterstellt sind. Wer hingegen auf dem eigenen Rezeptformular Arzneimittel verschreibt, übt damit seinen Beruf eigenverantwortlich aus; dafür braucht es nach kantonalem Gesundheitsgesetz einen Weiterbildungstitel und die Berufsausübungsbewilligung.

\section{Betäubungsmittel}

Betäubungsmittel, die für medizinische Verwendungen bestimmt sind, unterstehen speziellen Kontrollen. Der Hauptzweck der Vewendung muss medizinisch gerechtfertigt sein (beispielsweise die Behandlung von Schmerzen), und das spezifische Risiko der Abhängigkeit muss entsprechend berücksichtigt werden. Solche Produkte dürfen nur für einen begrenzten Zeitraum verordnet werden (in der Regel während eines Monats, in Ausnahmefällen bis maximal drei Monate pro Verordnung).

Psychotrope Arzneimittel (Benzodiazepine und ähnliche Produkte) unterstehen ebenfalls dem Betäubungsmittelgesetz. Solche Produkte dürfen nur für einen begrenzten Zeitraum verordnet werden (in der Regel während eines Monats, in Ausnahmefällen bis maximal sechs Monate pro Verordnung).

Die zeitlich beschränkte Gültigkeit der Verordnung von Betäubungsmitteln und psychotropen Substanzen ist darauf ausgerichtet, die medizinische Kontrolle durch den verordnenden Arzt zu gewährleisten. Sofern es angesichts der jeweiligen Umstände gerechtfertigt ist, kann der Arzt eine neue Verordnung mit der gleichen Dauer ausstellen.

Betäbungsmittel der Verzeichnisse a und d der BetmVV-EDI müssen auf den amtlichen BM-Rezeptformularen verordnet werden, pro Formular dürfen maximal 3 Betäubungsmittel verordnet werden. Für die Verschreibung, die Abgabe und die Verabreichung von Betäubungsmitteln (betrifft alle Verzeichnisse, somit auch Benzodiazepine) zur Behandlung von betäubungsmittelabhängigen Personen braucht es eine kantonale Bewilligung.

Ein Arzt, der als Arzneimittel zugelassene Betäubungsmittel für eine andere als die zugelassene Indikation abgibt oder verordnet, muss dies der zuständigen kantonalen Behörde melden. Auf Verlangen der Behörde ist die Abgabe oder Verordnung zu begründen.

\section{Medizinprodukte}

Medizinprodukte, d.h. «Produkte, einschliesslich Instrumente, Apparate, Invitro-Diagnostika, Software und andere Gegenstände oder Stoffe, die für die medizinische Verwendung bestimmt sind oder angepriesen werden und deren Hauptwirkung nicht durch ein Arzneimittel erreicht wird», ${ }^{61}$ müssen bestimmte 
Qualitätsnormen erfüllen. Im Gegensatz zu den Arzneimitteln stellt Swissmedic keine Zulassungen für solche Produkte aus. Die Unternehmen, die Medizinprodukte vermarkten, müssen nachweisen, dass diese den Anforderungen entsprechen und die geforderten Eigenschaften aufweisen. Die entsprechenden Zertifikate werden von für Medizinprodukte akkreditierten und benannten Konformitätsbewertungsstellen ausgestellt.

Wie bei den Arzneimitteln entscheidet das BAG über die Kassenpflicht der Medizinprodukte (vgl. Kap. 4.14.).

\section{7. "Off label use» und "unlicensed use»}

"Off label use» oder "unlicensed use» sind im Heilmittelgesetz nicht geregelt, aber als Teil der therapeutischen Freiheit des Arztes grundsätzlich zulässig.

Wenn ein Arzneimittel in der Schweiz zugelassen ist, aber der ärztlich verordnete Einsatz von der genehmigten Fachinformation abweicht, liegt ein sogenannter «off label use» vor. Meist wird das Arzneimittel dann bei einer anderen Indikation oder mit einer anderen Dosis verwendet. In vielen Fachbereichen (insbesondere Pädiatrie, Gynäkologie, Onkologie und Geriatrie) ist der «off label use» häufig, weil viele Medikamente für diese Patientengruppen nicht hinreichend untersucht und daher auch nicht ausdrücklich für sie zugelassen wurden. «Off label use» kann auch deshalb geschehen, weil die pharmazeutische Industrie die entsprechenden Anwendungen nicht immer zusätzlich registrieren lassen kann oder will, etwa in Ermangelung eindeutiger klinischer Daten, zufolge geringen Marktpotenzials, aus Angst vor Haftpflichtverfahren oder Imagerisiken ${ }^{62}$ oder um zu vermeiden, dass das BAG den Preis aufgrund der breiteren Indikationen senkt.

«Off label use» ist Teil der ärztlichen therapeutischen Freiheit und damit zulässig, auch wenn er im Heilmittelgesetz nicht erwähnt wird. Allerdings trägt der Arzt die Verantwortung für die Abweichung: Er muss begründen können, dass der «off label use» dem Stand der Wissenschaft entsprach, und hat sich im Rahmen seiner Sorgfaltspflicht nach Art. $3 \mathrm{HMG}$ und Art. $26 \mathrm{HMG}$ zu richten. Zudem muss er darauf hinweisen, dass bei «off label use» krankenversicherungsrechtlich eine Kostenübernahmepflicht nur unter engen Voraussetzungen Platz greift (Siehe Kapitel 4.14.).

Von einem «unlicensed use» spricht man, wenn es um die Verwendung eines Arzneimittels geht, das in der Schweiz nicht zugelassen ist, grundsätzlich aber der Zulassungspflicht unterliegen würde. Der «unlicensed use» kann ausnahmsweise zulässig sein (Art. 20 HMG in Verbindung mit Art. 36 Arzneimittel-Bewilligungsverordnung, AMBV). In der Regel ist dafür eine Bewilligung von Swissmedic

62 So hat z.B. der Hersteller von RU 486 das Medikament in vielen Ländern aus Imagegründen lange nicht registriert. 
erforderlich. Keine Sonderbewilligung ist nötig, wenn der Arzt ein Arzneimittel verschreibt, das von einem Land zugelassen wurde, dessen Zulassung als gleichwertig mit der Schweiz anerkannt wird (z.B. EU-Staaten oder USA).

\subsection{Nichtetablierte Behandlungen bei einzelnen Patienten}

Von einer nichtetablierten Behandlung spricht man z.B. in einer Situation, in der die Nutzenbewertung eines Arzneimittels unter Alltagsbedingungen noch aussteht. Für die Abgrenzung von Forschung und Behandlung spielt das Ziel der Intervention eine zentrale Rolle. Werden verallgemeinerbare Erkenntnisse angestrebt, die über den individuellen Entscheid der hier und jetzt durchgeführten Behandlung bei einem einzelnen Patienten hinausgehen, handelt es sich um Forschung. Nichtetablierte Behandlungen kommen insbesondere zur Anwendung,

- wenn für eine bestimmte Indikation bislang keine Standardbehandlung zur Verfügung steht; oder

- wenn die Standardbehandlung bei einem Patienten nicht oder nicht mehr hilft.

Wird eine nichtetablierte Behandlung nach Abwägung aller Standardverfahren primär vom Behandlungsinteresse für den einzelnen Patienten geleitet, liegt kein typischer Forschungsansatz vor. Die Übergänge zwischen nichtetablierter Behandlung und Forschung können allerdings fliessend sein. Die SAMW arbeitet deshalb medizin-ethische Richtlinien zur Konkretisierung der Sorgfaltspflicht in dieser speziellen Situation aus.

\subsection{Röntgenuntersuchungen und Labor}

Röntgenuntersuchungen setzen eine spezifische Ausbildung voraus. Für das

Praxislabor ist Qualitätssicherung mit externen Ringversuchen vorgeschrieben.

\section{Röntgen}

Im Strahlenschutzgesetz wird bei medizinischen Anwendungen zwischen dem Sachverstand und der Sachkunde unterschieden:

- Der Sachverstand ist eine Voraussetzung, um eine Röntgenanlage betreiben zu dürfen. Man erwirbt diesen mit dem Besuch eines durch das BAG anerkannten Strahlenschutzkurses. In einer Gruppenpraxis bzw. in einem Spital muss allerdings nur eine Person über diesen Sachverstand verfügen; das kann einer der Partnerärzte, aber beispielsweise auch eine Fachfrau für medizinischtechnische Radialogie (MTRA) sein. 
- Das eidgenössische Arztdiplom berechtigt nur zum Röntgen von Extremitäten und des Thorax. Für dosisintensive Röntgenanwendungen braucht es eine zusätzliche Weiterbildung, die sogenannte Sachkunde. Diese Sachkunde muss - im Gegensatz zum Sachverstand - auch in einer Gruppenpraxis jeder einzelne Arzt nachweisen können, sofern er entsprechende Röntgenuntersuchungen durchführen will. Voraussetzung für den Erwerb der Sachkunde ist in jedem Fall der Nachweis eines absolvierten Strahlenschutzkurses des BAG. In einzelnen Fachgebieten wird die Sachkunde direkt mit dem Facharzttitel erworben, andernfalls über einen eigenständigen Fähigkeitsausweis. ${ }^{63}$

Medizinische Praxisassistentinnen (MPA) mit eidgenössischem Fähigkeitszeugnis sind zum Röntgen von Extremitäten und des Thorax berechtigt. Für die Durchführung von dosisintensiven Aufnahmen muss die MPA eine zusätzliche, vom BAG anerkannte Weiterbildung absolvieren.

Gemäss Strahlenschutzverordnung ist der Inhaber einer Betriebsbewilligung verpflichtet, alle in seinem Betrieb tätigen strahlenexponierten Personen einer Personendosimetriestelle zu melden, die in regelmässigen Abständen die Strahlenexposition der einzelnen Arbeitnehmer ermittelt. Personen unter 16 Jahren dürfen nicht als beruflich strahlenexponierte Personen beschäftigt werden. ${ }^{64}$

\section{Labor}

Wer ein Praxislabor betreibt, muss eine Qualitätssicherung mit internen Kontrollen und externen Ringversuchen vorweisen können. Ob für das Praxislabor darüber hinaus eine spezifische Weiterbildung und damit der Erwerb des bereits angebotenen entsprechenden Fähigkeitsausweises des KHM verlangt werden soll, wird derzeit diskutiert. ${ }^{65}$ Für die Betriebsbewilligung für Grosslabors gelten zusätzliche Bestimmungen. Bestimmungen werden auf der Website des SIWF nachgeführt und erläutert: www.fmh.ch/bildung-siwf/weiterbildung_allgemein/roentgen.html

64 Strahlenschutzverordnung $\rightarrow$ www.admin.ch/ch/d/sr/c814_501.html

65 Die DV der FMH sprach sich 2011 für ein Obligatorium aus. Die jeweils geltenden Bestimmungen werden auf der Website des SIWF unter www.siwf.ch $\rightarrow$ Fähigkeitsprogramme $\rightarrow$ Praxislabor nachgeführt. 


\subsection{Grundprinzipien der obligatorischen Krankenpflegeversicherung}

In der obligatorischen Krankenpflegeversicherung ist die gesamte Wohnbevölkerung versichert. Anders als beispielsweise in der Unfallversicherung ist in der obligatorischen Krankenpflegeversicherung nicht relevant, weshalb jemand erkrankt ist. Die Krankenversicherung kommt hingegen nur zum Tragen, wenn nicht eine andere Sozialversicherung zuständig ist.

\section{Versicherungsobligatorium}

Wer seinen Wohnsitz in der Schweiz hat, muss sich in der obligatorischen Krankenpflegeversicherung (OKP) versichern, und dies spätestens drei Monate nach Wohnsitznahme oder Geburt. Damit gilt das Versicherungsobligatorium auch für hier lebende Ausländerinnen und für Saisonniers. Grenzgänger können sich gemäss KVG versichern, müssen aber nicht. Die Versicherungspflicht gilt ebenfalls für Asylbewerber während laufendem Verfahren (BGE 129 V $77^{66}$ ), doch berät das Parlament über eine allfällige Verschärfung des Asylgesetzes. ${ }^{67}$ Nicht mehr obligatorisch bei einer Krankenkasse versichert sind illegal Anwesende (sogenannte $\mathrm{NEE}^{68}$ ) und abgewiesene Asylbewerber.

Sowohl das Freizügigkeitsabkommen zwischen der Schweiz und der EU ${ }^{69}$ als auch das Übereinkommen mit der EFTA ${ }^{70}$ regeln die Behandlung von Aufenthaltern (z.B. Touristen): Wenn sie die europäische Krankenversicherungskarte (EKVK) oder die provisorische Ersatzbescheinigung vorlegen, sind sie wie Krankenversicherte aus der Schweiz zu behandeln, d.h., sie haben Anrecht auf Tarifschutz.

Die Selbstbehandlung des Arztes ist gemäss Bundesgericht nicht kassenpflichtig, und auch sich selbst verschriebene Medikamente werden von der Kasse nicht übernommen. Wenn hingegen eine Ärztin ihre Familienangehörigen behandelt, so ist diese Behandlung kassenpflichtig. ${ }^{71}$

66 E.5.2.: "Die dem Obligatorium unterworfenen Personen ohne Aufenthaltsbewilligung, aber mit Wohnsitz in der Schweiz, bezahlen ebenso Krankenkassenprämien und ihr Einkommen unterliegt ebenfalls der Steuerpflicht, sodass sie auch den staatlich subventionierten Teil der Krankenpflegeversicherung nach Massgabe ihrer Einkommensverhältnisse mitfinanzieren.»

67 www.parlament.ch/d/suche/seiten/ratsunterlagen.aspx?gesch_nr=20100052, www.parlament.ch/ab/frameset/d/n/4904/384306/d_n_4904_384306_384317.htm (Diskutiert wird zurzeit der Wechsel von Sozialhilfe zu blosser Nothilfe während der Dauer des Asylverfahrens; dies soll nicht gelten für besonders Schutzbedürftige wie beispielsweise Familien mit Kindern.)

68 Nichteintretensentscheide.

69 Die neuen EU-Verordnungen Nr. 883/2004 und 987/2009 sind zwischen der Schweiz und den EU-Mitgliedstaaten seit dem 1. April 2012 anwendbar. An diesem Tag trat der revidierte Anhang II zum Freizügigkeitsabkommen (FZA) in Kraft. Diese Verordnungen ersetzen die Verordnungen Nr. 1408/71 und 574/72.

70 Zwischen der Schweiz und den EFTA-Staaten (Island, Liechtenstein, Norwegen) bleiben die Verordnungen Nr. 1408/71 und 574/72 in Kraft. BGE $125 \vee 430$. 
Die obligatorische Krankenpflegeversicherung gemäss Krankenversicherungsgesetz (KVG) ist eine sogenannt finale Versicherung: Das heisst, es kommt nicht darauf an, weshalb jemand krank geworden ist; es gibt daher auch keine Kürzung bei Selbstverschulden. Die Behandlungskosten werden von der Krankenkasse aber nur übernommen, wenn keine andere Sozialversicherung (UVG, MVG, IVG) zuständig ist. Dafür werden auch die Unfallbehandlungen durch den Krankenversicherer nach den KVG-Regeln ${ }^{72}$ übernommen, wenn der Patient keine UVGVersicherung hat.

Die OKP ist vorleistungspflichtig im Verhältnis zu den anderen Sozialversicherungen: Wenn die Leistungspflicht gemäss UVG, MVG oder IVG umstritten ist, zahlt die Krankenkasse nach den Regeln des KVG bis zur Klärung des Falls. ${ }^{73}$

\section{Kostenübernahmeprinzip}

In Artikel 25 des KVG heisst es, dass die OKP die Kosten für jene Leistungen übernimmt, die der Diagnose oder Behandlung einer Krankheit und ihrer Folgen dienen. Es gilt also das Kostenübernahmeprinzip. Die Behandlungsführung und -verantwortung liegt damit bei der behandelnden Ärztin, und nicht beim Versicherer.

Es gibt allerdings Ausnahmen von diesem Prinzip: Der Bundesrat kann namentlich bei «besonders kostspieligen Diagnose- oder Behandlungsverfahren die vorgängige Zustimmung des Vertrauensarztes» verlangen. ${ }^{74}$ Dies tut der Bundesrat denn auch immer häufiger.

\section{Pflichtleistung und Indikation im KVG}

Das Gesetz definiert Krankheit als «jede Beeinträchtigung der körperlichen, geistigen oder psychischen Gesundheit, die nicht Folge eines Unfalls ist und die eine medizinische Untersuchung oder Behandlung erfordert oder eine Arbeitsunfähigkeit zur Folge hat». ${ }^{75}$ Entscheidend für die Frage, ob eine Behandlung von der Krankenversicherung bezahlt wird, ist damit grundsätzlich die medizinische Indikation. Damit liegt die Definitionsmacht für die Leistungen der Krankenversicherung primär in der Medizin. ${ }^{76}$

\footnotetext{
D.h. zum Kassentarif und mit Franchise und Selbstbehalt.

Art 70 ATSG. (www.admin.ch/ch/d/sr/830_1/a70.html)

Art. 58 KVG.

Art. 3 ATSG.

76 Anderes gilt in UVG, MVG, IVG und Taggeldversicherung sowohl für die Entstehung des Leistungsanspruchs überhaupt wie auch für Taggeld- oder Rentenleistungen.
} 
Für alle vom Arzt oder von der Ärztin durchgeführten Untersuchungen oder Behandlungen gilt die Vermutung, dass sie Pflichtleistungen sind. Das Departement des Innern hat nach Anhörung der Eidgenössischen Kommission für allgemeine Leistungen und Grundsatzfragen (Leistungs- und Grundsatzkommission) verschiedene ärztliche Pflichtleistungen in der Krankenpflege-Leistungsverordnung (KLV) und im Anhang 1 dazu näher definiert. Die KLV ist damit weder eine Positiv- noch eine Negativliste, sondern eine blosse Sammlung von Entscheiden bei umstrittenen Leistungen.

Wie das KVG in Artikel 33 vorschreibt, müssen die Untersuchungen und Behandlungen wirksam, zweckmässig und wirtschaftlich sein. Sie müssen zudem auf das begrenzt werden, was «im Interesse des Versicherten liegt und für den Behandlungszweck erforderlich ist». ${ }^{77}$ Wirksam sind die Massnahmen, wenn ihre Wirksamkeit gemäss dem Stand des medizinischen Wissens nachgewiesen ist. Zweckmässig bedeutet, dass die Untersuchung oder Behandlung auf den konkreten Fall angepasst ist und dass sich ein Heilungserfolg erwarten lässt. Wirtschaftlich heisst: Stehen zwei gleichermassen zweckmässige Behandlungen zur Auswahl, muss die kostengünstigere Variante gewählt werden; gibt es hingegen keine echte Alternative, kann nur ein grobes Missverhältnis zwischen Kosten und Nutzen die Leistungsverweigerung rechtfertigen. ${ }^{78}$

\subsection{Leistungserbringer und Wahlfreiheit im KVG}

\section{Das Krankenversicherungsgesetz legt fest, welche Leistungserbringer über die obligatorische Krankenpflegeversicherung abrechnen können. In der Wahl dieser Leistungserbringer sind die Versicherten aber grundsätzlich frei.}

Das Gesetz definiert, welche Leistungserbringer für das KVG zugelassen sind:

- Ärzte sowie ambulante Einrichtungen der ärztlichen Krankenpflege, Apotheker, Zahnärztinnen, Chiropraktoren und Hebammen;

- Spitäler, Geburtshäuser, Pflegeheime und Heilbäder;

- Personen, die auf ärztliche Anordnung hin Leistungen erbringen (Paramedics), oder Organisationen, die solche Personen beschäftigen: Physiotherapeuten, Ergotherapeutinnen und Ergotherapieorganisationen, Pflegefachleute, Logopäden, Ernährungs- und Diabetesberaterinnen, Spitexorganisationen;

- Abgabestellen für Mittel und Gegenstände, Transport- und Rettungsunternehmen sowie Labors. 
Wer die staatlichen Zulassungsvoraussetzungen erfüllt, hat Anspruch auf eine ZSR-Nummer der SASIS AG. ${ }^{79}$

\section{Wahl des Leistungserbringers und Kostenübernahme im ambulanten Bereich}

Die Versicherten können unter den zugelassenen Leistungserbringern, die für die ambulante Behandlung geeignet sind, frei wählen. ${ }^{80}$ Der Krankenversicherer übernimmt die Kosten maximal nach dem Tarif, der am Wohn- oder Arbeitsort der versicherten Person oder in deren Umgebung gilt. Fährt der Patient ohne medizinische Gründe ${ }^{81}$ für eine ambulante Behandlung zu weit von seinem Wohnort weg, muss er die allfällige Tarifdifferenz zwischen Behandlungsort und Wohnort bezahlen. Für die Frage, was zu weit weg ist, gilt bei ambulanten Behandlungen nicht die Kantonsgrenze, sondern ein spezieller Umgebungsbegriff. Die massgebliche Umgebung ist grösser oder kleiner je nach Anzahl der ambulanten Leistungserbringer im Fachgebiet. Liegen hingegen medizinische Gründe vor für eine Behandlung bei einem weiter entfernten Leistungserbringer, so richtet sich die Kostenübernahme nach dem Tarif, der für diesen Leistungserbringer gilt. ${ }^{82}$

\section{Wahl des Leistungserbringers und Kostenübernahme im stationären Bereich}

Im Spital hat die Patientin in der Grundversicherung zwar keine freie Arztwahl. Für die stationäre Behandlung akuter Krankheiten (Somatik oder Psychiatrie) oder der stationären Durchführung von Massnahmen der medizinischen Rehabilitation können die Versicherten aber seit Einführung der neuen Spitalfinanzierung ${ }^{83}$ unter jenen Spitälern frei wählen, die auf der Spitalliste ihres Wohnkantons oder jener des Standortkantons aufgeführt sind (Listenspital). ${ }^{84}$ Dasselbe gilt sinngemäss für Geburtshäuser. Die Listenspitäler gelten als zugelassen zur Tätigkeit zulasten der obligatorischen Krankenpflegeversicherung (OKP). Der Versicherer und der Wohnkanton übernehmen die Vergütung bei stationärer Behandlung in einem Listenspital anteilsmässig ${ }^{85}$, jedoch - ausser es sei medizinisch

www.sasis.ch; Das Bundesgericht hat den Rechtsanspruch auf Erteilung einer ZSR-Nummer gestützt auf die vertragliche Verpflichtung bejaht, mit der ZSR-Nummer abzurechnen. (BGE 132 V 303 vom 27. März 2006, K 139/04).

80 Art. 41 Abs. 1 KVG.

81 Medizinische Gründe liegen vor bei einem Notfall oder wenn die erforderlichen Leistungen nicht angeboten werden: bei ambulanter Behandlung am Wohn- oder Arbeitsort der versicherten Person oder in deren Umgebung (Art. 41 Abs. 3bis lit. a KVG).

82 Art. 41 Abs. 2 KVG.

83 Gesetzesrevision in Kraft seit 1.1.2009, eingeführt per 1.1.2012.

84 Art. 41 Abs. 1 bis KVG.

85 Art. 41 Abs. 1 bis KVG i.V.m. Art. 49a Abs. 1 und 2 KVG; der Kantonsanteil beträgt mindestens 55 Prozent. 
notwendig ${ }^{86}$ - nur nach dem Tarif, der in einem Listenspital des Wohnkantons für die betreffende Behandlung gilt (Referenztarif). ${ }^{87}$

Beansprucht die versicherte Person bei einer stationären Behandlung aus medizinischen Gründen ein nicht auf der Spitalliste des Wohnkantons aufgeführtes ausserkantonales Listenspital, so übernehmen die Versicherung und der Wohnkanton die Vergütung auch anteilsmässig. Soll die Behandlung zum Tarif des behandelnden Spitals erfolgen, braucht es - ausser im Notfall - eine Bewilligung (Kostengutsprache) des Wohnkantons. ${ }^{88}$

Neben den Listenspitälern gibt es in der Grundversicherung noch die Kategorie der Vertragsspitäler. Diese sind zwar zur Leistungserbringung nach KVG zugelassen, befinden sich aber weder auf der Liste des Wohnort- noch des Standortkantons. Die Vergütung erfolgt bei den Vertragsspitälern aufgrund eines Vertrages zwischen den Versicherern und den Spitälern, wobei die Versicherer nicht einen höheren Anteil aus der Grundversicherung zahlen dürfen als bei den Listenspitälern. Der Rest der Kosten der Behandlung geht zulasten der Zusatzversicherung oder muss vom Patienten selbst getragen werden. Der Kanton beteiligt sich hier nicht.

\subsection{Managed Care}

\section{Der Versicherte kann im Versicherungsvertrag freiwillig auf die volle Arzt- und Spitalwahlfreiheit verzichten und eine Ärztin oder einen Arzt als Care Manager wählen. Asylsuchende müssen in Managed-Care-Modellen versichert sein.}

Mit der Möglichkeit von Managed-Care-Modellen wollte der Gesetzgeber mit dem KVG von 1994 Kosten senken. So heisst es im Krankenversicherungsgesetz (KVG): «Die Versicherten können [...] ihr Wahlrecht auf Leistungserbringer beschränken, die der Versicherer im Hinblick auf eine kostengünstigere Versorgung auswählt.» ${ }^{89}$

Aus Sicht der FMH basiert Managed Care auf Zusammenarbeitsverträgen zwischen Netzwerken und Versicherern, die «insbesondere die Zusammenarbeit, den Datenaustausch, die Qualitätssicherung und die Vergütung der Leistungen» ${ }^{90}$ regeln. nicht angeboten werden: bei stationärer Behandlung in einem Spital, das auf der Spitalliste des Wohnkantons aufgeführt ist (Art. 41 Abs. 3bis lit. b KVG).

87 Thomas Plattner et al, Für Patienten steht viel auf dem Spiel - auch nach dem 1. Januar 2012, Schweizerische Ärztezeitung 2011; 92: 41, 1585-87.

88 Art. 41 Abs. 3 KVG.

89 Art. 41 Abs. 4 KVG.

90 FMH-Thesen zu Managed Care, 2006 (SÄZ 36/2006; S.1535ff). 
Am 17. Juni 2012 haben die Stimmbürger eine Managed Care-Vorlage mit 76\% Nein-Stimmen abgelehnt, die insbesondere eine obligatorische Budgetmitverantwortung der Ärzte im Netzwerk, tiefere Selbstbehalte für Patienten im Netzwerk sowie eine Verbesserung des Risikoausgleichs beinhaltet hätte. Die Versicherer hätten weiterhin wie im geltenden KVG ergänzend zu Netzwerken einseitig definierte Listenmodelle anbieten können. Die Weiterentwicklung ist bei Redaktionsschluss des Leitfadens offen; Konsens scheint für eine Verbesserung des Risikoausgleichs zu bestehen. ${ }^{91}$

\subsection{Qualitätssicherung und Register}

Das Krankenversicherungsgesetz verpflichtet Ärztinnen und Ärzte, qualitätssichernde Massnahmen zu ergreifen. Register sind ein wichtiges Instrument der Qualitätssicherung und Forschung. Zertifizierung und Akkreditierung erlauben dem Staat, gewisse Qualitätskontrollen und Zulassungen an Dritte zu delegieren.

Das KVG verlangt Qualitätssicherung. ${ }^{92}$ Die Verordnung über die Krankenversicherung (KVV) sieht vor, dass «die Leistungserbringer oder deren Verbände Konzepte und Programme über die Anforderungen an die Qualität der Leistungen und die Förderung der Qualität» erarbeiten. «Die Modalitäten der Durchführung werden in den Tarifverträgen oder in besonderen Qualitätssicherungsverträgen mit den Versicherern oder deren Verbänden vereinbart. Die Bestimmungen haben den allgemein anerkannten Standards zu entsprechen, unter Berücksichtigung der Wirtschaftlichkeit der Leistungen.» (Art. 77 KVV). Seit 2009 ist ein revidierter Art. 22a KVG in Kraft, der die Leistungserbringer «verpflichtet, den zuständigen Bundesbehörden die Daten bekannt zu geben, die benötigt werden, um die Anwendung der Bestimmungen dieses Gesetzes über die Wirtschaftlichkeit und Qualität der Leistungen zu überwachen».

2009 hat die SAMW Empfehlungen zur Erhebung, Analyse und Veröffentlichung von Daten über die medizinische Behandlungsqualität veröffentlicht. Qualitätssicherung dient der Transparenz und dem Qualitätswettbewerb; die Transparenz soll aber nicht dazu führen, dass Spitäler aus Sorge um ihre Statistik Risikopatienten abweisen. Entscheidend sind Relevanz, Korrektheit und Verständlichkeit der Daten.

92 Art. 43 Abs. 2 lit. d und Art. 58 KVG. In den übrigen Sozialversicherungen fehlt eine entsprechende explizite gesetzliche Grundlage für OS. Botschaft KVG BBI 1991 S.129: "Qualitätssicherung trägt zur Kostendämpfung bei, indem sie Ressourcen von den Tätigkeiten abzieht, die unnötig, unwirksam und unzweckmässig sind. Der Begriff der Qualität wird weit gefasst, damit die drei Elemente, Behandlungsergebnisse, Angemessenheit der Leistung und Zufriedenheit des Patienten, von der Qualitätskontrolle erfasst werden können (vgl. Art. 50).» 
Es gibt einzelne Bereiche mit expliziten Qualitätssicherungsverträgen, beispielsweise für das Labor (Qualab) oder für die Zertifizierung von Operationssälen gemäss TARMED. Diese Verträge gelten für alle Sozialversicherungen (nicht nur für den Bereich KVG).

Ein wichtiges Element der Qualitätssicherung ist die offene Diskussion von Fällen und Problemen in Qualitätszirkeln. Eine weitere Anwendung ist das «Critical Incident Reporting System» (CIRS), das heisst die Auswertung von kritischen Ereignissen ohne eigentliche Schadenfolge: Wer Schadenfälle wirksam vermeiden will, konzentriert sich auf die Reduktion der Anzahl unsicherer Handlungen. Organisatorische und technische Vorkehrungen sind dabei zentral. Meldungen von «critical incidents» sollen aus Datenschutzgründen bei der Auswertung anonymisiert, und die Originalmeldung soll vernichtet werden. ${ }^{93}$

\section{Klinische und epidemiologische Register}

In einem klinischen Register werden typischerweise die Patienten erfasst, die in einer oder in wenigen Institutionen behandelt werden. Typische Auswertungsfragen sind die nach Einflussfaktoren (wie Alter, Geschlecht oder einzelne Befunde) für den Erfolg einer Therapie oder ganz allgemein für die Prognose des Patienten, sowie Fragen nach Häufigkeit des Auftretens (Inzidenz) einer Komplikation oder eines anderen unerwünschten Ereignisses.

Epidemiologische Register versuchen, eine bestimmte Region (z.B. einen Kanton oder auch das Gebiet der ganzen Schweiz) möglichst vollzählig zu erfassen. Sie dienen meist der Erforschung einer bestimmten, meist schweren und oft seltenen Krankheit. Eine typische Fragestellung ist die nach dem Auftreten (Inzidenz) oder der Verbreitung (Prävalenz) einer Krankheit in der Region (bzw. den Regionen), gegliedert nach Differentialdiagnosen, Geschlecht, Altersklasse usw., sowie das Erkennen einer zunehmenden (z.B. von Leukämien in der Nähe von Atomkraftwerken) oder abnehmenden (nach Impfkampagnen) Inzidenz oder Prävalenz.

Klinische und epidemiologische Register arbeiten in der Regel mit Daten, die dem Arztgeheimnis unterstehen, und benötigen deshalb entweder die Zustimmung des Patienten oder eine gesetzliche Grundlage. Viele Kantone haben gesetzliche Grundlage für Krebsregister geschaffen.

Der Bund arbeitet an einem Entwurf für ein Krebsregistergesetz des Bundes. Es soll als Rahmengesetz sowohl für epidemiologische als auch klinische Register dienen und ebenfalls Register regeln, die nicht Krebskrankheiten betreffen. 


\section{Abgrenzung von Qualitätssicherung und Forschung}

Die Unterscheidung zwischen Qualitätssicherung und Forschung ist wichtig, weil Qualitätssicherungsprogramme gemäss KVG von den Krankenkassen zu finanzieren sind, Forschung hingegen nicht. Das Bundesgericht definierte Forschung gemäss Heilmittelgesetz so breit, dass darunter auch fällt, was gemäss KVG noch Qualitätssicherung wäre. ${ }^{94}$ Gemäss Botschaft zum Humanforschungsgesetz sollen Qualitätskontrollstudien künftig dann als Forschung gelten, «wenn zusätzlich zur Qualitätskontrolle methodengeleitet neue, verallgemeinerbare Erkenntnisse gewonnen werden sollen».

\section{Akkreditierung im akademischen Bereich - Zertifizierung von Spitälern oder Praxen}

Die Anerkennung von Studiengängen der Fakultäten oder Weiterbildungsprogrammen des SIWF wird Akkreditierung genannt. Im Medizinalberufegesetz MedBG wird die Akkreditierung folgendermassen definiert: «Die Akkreditierung hat den Zweck zu überprüfen, ob es die Aus- und Weiterbildungsgänge den Personen in Aus- und Weiterbildung erlauben, die Ziele dieses Gesetzes zu erreichen. Sie schliesst die Überprüfung der Qualität von Strukturen, Prozessen und Ergebnissen ein.» (Art. 22 MedBG; siehe auch Kap. 7.3.)

Nicht nur Spitäler, sondern auch verschiedene Arztpraxen haben hingegen eine freiwillige Qualitätszertifizierung durchlaufen; dies ist eine Form der Qualitätssicherung, die sich in der Regel auf die Infrastruktur- und die Prozessqualität bezieht. Die gesetzliche Grundlage dafür liefert das Bundesgesetz über die technischen Handelshemmnisse (THG). Zertifizierungen können sowohl Qualitäts- wie Werbeinstrument sein. Im Bereich des THG bedeutet Akkreditierung die Zulassung eines Zertifizierers. ${ }^{95}$ 


\subsection{Kostenübernahme durch die Krankenpflege- versicherung von Arzneimitteln, Mitteln und Gegen- ständen im KVG}

\section{Kassenpflichtige Arzneimittel, Mittel und Gegenstände gemäss Positivlisten}

Kosten für Leistungen, die der Diagnose oder Behandlung einer Krankheit und ihrer Folgen dienen, werden von der obligatorischen Krankenpflegeversicherung übernommen. Diese Leistungen umfassen unter anderem die ärztlich verordneten Analysen und Arzneimittel sowie die der Untersuchung oder Behandlung dienenden Mittel und Gegenstände. ${ }^{96}$

Das Departement erlässt nach Anhören der zuständigen Kommissionen

- die Arzneimittelliste mit Tarif (ALT), d.h. die Liste der in der Rezeptur verwendeten Präparate, Wirk- und Hilfsstoffe mit Tarif; dieser umfasst auch die Leistungen des Apothekers oder der Apothekerin;

- die Mittel- und Gegenstände-Liste (MiGeL), d.h. Bestimmungen über die Leistungspflicht und den Umfang der Vergütung bei Mitteln und Gegenständen, die der Untersuchung oder Behandlung dienen. Die MiGeL enthält Höchstvergütungsbeträge. Lässt sich der Versicherte ein teureres Mittel verschreiben, muss er die Differenz zum Listenbetrag selbst tragen. ${ }^{97}$

Das Bundesamt erstellt

- die Spezialitätenliste (SL), d.h. die Liste der pharmazeutischen Spezialitäten und konfektionierten Arzneimittel mit Preisen. Diese Liste enthält auch die mit den Originalpräparaten austauschbaren preisgünstigeren Generika. ${ }^{98}$

Kassenpflichtig sind grundsätzlich Arzneimittel, Mittel und Gegenstände, die in einer dieser Positivlisten (ALT, MiGel, SL) aufgeführt sind.

Die Kosten eines auf der Spezialitätenliste aufgeführten Arzneimittels werden von der Krankenversicherung zudem grundsätzlich nur übernommen, wenn dieses für von Swissmedic genehmigte medizinische Indikationen verschrieben wurde. ${ }^{99}$ 
"Off label use" und "unlicensed use"

Wird ein in der Spezialitätenliste aufgeführtes Arzneimittel ausserhalb der zugelassenen Indikation oder Dosierung abgegeben, so spricht man von «off label use». Beim «unlicensed use» ${ }^{100}$ wird ein Arzneimittel eingesetzt, das in der Schweiz nicht zugelassen ist, grundsätzlich aber der Zulassungspflicht nach Artikel 9 Absatz 1 HMG unterliegt. ${ }^{101}$

Die vom Bundesgericht entwickelte Praxis zum «off label use» wurde mehr oder weniger in Art. 71a und b KVV übernommen; die revidierte KVV trat am 1. März 2011 in Kraft.

Die obligatorische Krankenpflegeversicherung übernimmt unter bestimmten Voraussetzungen die Kosten eines in die Spezialitätenliste aufgenommenen Arzneimittels für eine Anwendung ausserhalb der vom Institut genehmigten Fachinformation oder ausserhalb der in der Spezialitätenliste festgelegten Limitierung. Unter denselben Voraussetzungen übernommen werden die Kosten für ein vom Institut zugelassenes verwendungsfertiges Arzneimittel, das nicht in die Spezialitätenliste aufgenommen ist für eine Anwendung innerhalb oder ausserhalb der Fachinformation.

Gemäss Art. 71a Abs. 1 Bst. a oder b KVV werden die Kosten für die erwähnten Arzneimittel übernommen, wenn

- der Einsatz des Arzneimittels eine unerlässliche Voraussetzung für die Durchführung einer anderen von der obligatorischen Krankenpflegeversicherung übernommenen Leistung bildet und diese eindeutig im Vordergrund steht (sogenannter Behandlungskomplex); oder

- vom Einsatz des Arzneimittels ein grosser therapeutischer Nutzen gegen eine Krankheit erwartet wird, die für die versicherte Person tödlich verlaufen oder schwere und chronische gesundheitliche Beeinträchtigungen nach sich ziehen kann, und wegen fehlender therapeutischer Alternativen keine andere wirksame und zugelassene Behandlungsmethode verfügbar ist.

Erforderlich ist neu eine vorgängige Kostengutsprache des Versicherers nach Konsultation des Vertrauensarztes oder der Vertrauensärztin. «Die zu übernehmenden Kosten müssen in einem angemessenen Verhältnis zum therapeutischen Nutzen stehen. Der Versicherer bestimmt die Höhe der Vergütung.» ${ }^{102}$ 
Wird ein vom Institut nicht zugelassenes Arzneimittel eingesetzt, das nach dem Heilmittelgesetz eingeführt werden darf, werden die Kosten nur übernommen, wenn die eben erwähnten Voraussetzungen nach Artikel 71a Abs. 1 Bst. a oder b KVV erfüllt sind und zusätzlich das Arzneimittel von einem Land mit einem vom Institut als gleichwertig anerkannten Zulassungssystem für die entsprechende Indikation zugelassen ist. ${ }^{103}$

\section{Generika und Co-Marketing-Arzneimittel}

Ein Generikum sollte sich gemäss KKV durch identische Wirkstoffe sowie die gleiche Darreichungsform und Dosierung wie das Originalpräparat auszeichnen. ${ }^{104}$ Hingegen können die Trägerstoffe, wie z. B. Bindemittel, Aromata, Stabilisatoren, Antioxidantien und Farbstoffe, verschieden sein, was Auswirkungen auf die Galenik und auch hinsichtlich Trägerstoff-assoziierter unerwünschter Arzneimittelwirkungen (u.a. Allergien) haben kann. ${ }^{105}$ Im Unterschied zum Generikum, das nur im Wesentlichen gleich ist wie ein Originalpräparat, ist das Co-MarketingArzneimittel einzig mit Ausnahme der Bezeichnung und Packung identisch mit dem Basispräparat. ${ }^{106}$

Der Arzt oder die Ärztin informiert den Patienten oder die Patientin, wenn in der Spezialitätenliste mindestens ein mit dem Originalpräparat austauschbares Generikum aufgeführt ist. ${ }^{107}$

Der Krankenversicherer erhebt einen Selbstbehalt von 20\%, wenn ein Originalmedikament verwendet wird, für das in der Spezialitätenliste austauschbare Generika aufgeführt sind, deren Höchstpreise mindestens 20\% tiefer sind als der Preis des Originalmedikamentes. Die Originalmedikamente mit einem Selbstbehalt von 20\% finden sich in einer aktualisierten Liste des Bundesamts für Gesundheit. ${ }^{108}$ Verschreibt der Arzt aus medizinischen Gründen ausdrücklich ein Originalpräparat, obwohl es ein billigeres Generikum gibt, beträgt der Selbstbehalt lediglich $10 \% .^{109}$

Apotheker oder Apothekerinnen können Originalpräparate der Spezialitätenliste durch die günstigeren Generika dieser Liste ersetzen, wenn nicht der Arzt oder die Ärztin ausdrücklich die Abgabe des Originalpräparates verlangt. Erfolgt eine Substitution, informieren sie die verschreibende Person über das abgegebene Präparat. ${ }^{110}$ Verlangt nun ein Arzt oder eine Ärztin ausdrücklich die Abgabe des Origi-

103 Art. 71b KVV.

104 64a Abs. 2 KVV.

105 Vgl. etwa Stephan Rüegg et al, Stellungnahme der Schweizerischen Liga gegen Epilepsie (SLgE),

Einsatz von Antiepileptika-Generika in der Epilepsietherapie, Schweiz. Ärztezeitung 49/2011

S. 1909-12.

106 Art. 64a Abs. 3 KVV.

107 Art. 38a Abs. 6 KLV.

108 http://bag.e-mediat.net/SL2007.Web.External/Default.aspx

109 www.bag.admin.ch/themen/krankenversicherung/04114/04285/index.html?lang=de

110 Art. 52a KVG. 
nalpräparates, muss dies auf dem Rezept handschriftlich und deutlich vermerkt werden: «aus medizinischen Gründen nicht substituieren». Dieser Hinweis zeigt der Apothekerin bzw. dem Apotheker an, dass eine Substitution ausgeschlossen ist. (Der Vermerk «sic» genügt nicht.) Der Arzt oder die Ärztin muss ausserdem auf der Rechnung einen Hinweis anbringen, dass die Verschreibung des Originalpräparates medizinisch begründet ist. ${ }^{111}$

\section{Gebot der Weitergabe von Vergünstigungen versus Verbot gezielter Einkaufspolitik}

Im Arzneimittelbereich setzt das BAG nur noch den Ex-Factory-Preis und den Höchstpreis für den Patienten fest. Zwischen Hersteller, Grossist und Detaillist soll hingegen der Markt spielen.

Gemäss Art. 33 im HMG dürf(t)en Rabatte den Entscheid, welche Medikamente abgegeben oder verschrieben werden, nicht beeinflussen - obschon Arzt, Ärztenetzwerk und Spital mit gezieltem Einkauf Kosten im Gesundheitswesen sparen können. Ziel des KVG ist hingegen eine günstige Versorgung bei guter Qualität. ${ }^{112}$ Gemäss Art. 56 Abs. 3 KVG darf der Leistungserbringer seinen Einkaufsentscheid durchaus auch vom Preis abhängig machen; er muss bloss den gewährten Rabatt oder die Vergünstigung an die Patienten bzw. die Krankenversicherer weitergeben. Hier kollidieren die Regeln des HMG 33 und des KVG 56 noch miteinander. Der Bundesrat schlägt in der aktuellen Revision des HMG vor, dass Rabatte künftig auch im HMG dann zulässig sind, wenn sie transparent gemacht und an die Konsumenten oder Versicherer weitergegeben werden. ${ }^{113}$

\subsection{Tarifrecht und Verfahren bei Streitigkeiten im KVG}

\section{Versicherer und Leistungserbringer legen die Tarife für die Leistungen in Verträgen fest. Diese müssen in der Folge von den Behörden genehmigt werden. Das KVG legt fest, wie bei Tarifstreitigkeiten zu verfahren ist.}

Einzelleistungstarife müssen auf einer gesamtschweizerischen Tarifstruktur beruhen (TARMED). ${ }^{114}$ Seit Januar 2012 müssen die Akutspitäler ihre stationären Behandlungen ebenfalls nach schweizweit einheitlichen Fallpauschalen (SwissDRG) abrechnen. ${ }^{115}$

111 Kommentar zu den Verordnungsänderungen vom 9. November und

12. Dezember 2005 des Bundesamts für Gesundheit.

112 Art. 43 KVG.

113 Art. 57b des HMG-Revisionsentwurfs vom 7. 11. 2012.

114 Art. 43 Abs. 5 KVG.

115 Art. 49 Abs. 1 KVG. 
Haben sich Versicherer und Leistungserbringer bzw. deren Verbände auf einen Tarifvertrag geeinigt, muss dieser vor dem Inkrafttreten von der Behörde genehmigt werden (je nach Tarifregion vom Bundesrat oder von der Kantonsregierung). ${ }^{116}$ Dies gilt auch für Managed-Care-Verträge ${ }^{117}$. Der Bundesrat hat aber schon ausnahmsweise - wie im Fall des Mammographie-Screenings in Genf - auf diesen Genehmigungsvorbehalt verzichtet, um nicht nachträglich ein Chaos hervorzurufen.

Falls kein Tarifvertrag zustande kommt, muss die genehmigende Behörde einen Ersatztarif festlegen. Wenn ein Arzttarifvertrag ausläuft, gilt für ein Jahr ein Rahmentarif; danach kann die Behörde den Tarif neu festsetzen. Seit Annahme der Parlamentarischen Initiative TARMED ist auch der Bundesrat subsidiär zur Tarifergänzung legitimiert. ${ }^{118}$

Für die Leistungen gemäss KVG müssen die vertraglich vereinbarten Tarife bzw. die behördlichen Ersatztarife eingehalten werden (Tarifschutz). ${ }^{19}$ Zusatzrechnungen sind aber zulässig für echte Mehrleistungen; zu den Mehrleistungen gehört zum Beispiel die Behandlung durch die erfahrene Ärztin im Spital. ${ }^{120}$

\section{Rechtsstreitigkeiten zwischen Patient und Krankenversicherer}

Über Leistungen, Forderungen und Anordnungen, die erheblich sind oder mit denen der Versicherte nicht einverstanden ist, muss der Krankenversicherer eine Verfügung mit Begründung und Rechtsmittelbelehrung erlassen. ${ }^{121}$ Der Versicherte kann gegen die Verfügung zur Sache selbst innert 30 Tagen Einsprache beim verfügenden Versicherer einreichen. Nächste Instanz ist das Kantonale Versicherungsgericht, das «rasch und für die Parteien kostenlos» entscheiden soll. Wo die Verhältnisse es rechtfertigen, wird der Anwalt vom Staat bezahlt. Letzte Instanz ist das Bundesgericht in Luzern.

117 Die im Juni 2012 abgelehnte Managed-Care-Vorlage hätte die Genehmigungspflicht für Managed-Care-Verträge aufgehoben.

118 Art. 43 Abs. 5bis KVG.

119 Art. 44 Abs. 1 KVG.

120 BGE 130 I 306, 9C_725/2008 vom 9.11.2009.

121 Art. 49 ATSG: Nicht zu verfügen hat der Sozialversicherer gegenüber dem - rechtlich ihm gleichgestellten - Leistungserbringer. 


\section{Rechtsstreitigkeiten zwischen Leistungserbringern und Krankenversicherer; Tarifergänzungen}

Streitigkeiten zwischen Versicherern und Leistungserbringer sind gemäss KVG durch ein kantonales Schiedsgericht zu regeln. ${ }^{122}$ Im ambulanten Bereich sehen praktisch alle Tarifverträge ${ }^{123}$ vor, dass vor Anrufung des Schiedsgerichts ein Schlichtungsverfahren vor einer Paritätischen Kommission (PVK) durchgeführt wird. Im stationären Bereich ist eine PVK nicht vorgeschaltet.

Im TARMED gibt es je nach Thema verschiedene paritätische Kommissionen:

Für Tarifinterpretationsfragen im TARMED ist quer über alle Sozialversicherungsgesetze zuerst die Paritätische Interpretationskommission (PIK) aller vier Vertragspartner für den Schlichtungsvorschlag zuständig. Konkrete Fälle sind an die TARMED Suisse zu richten, wobei eine Vorbesprechung mit dem FMH-Tarifdienst sinnvoll ist.

Für Fragen zur Dignität ${ }^{124}$ oder der Spartenanerkennung ist zuerst die Paritätische Kommission Dignität (PaKoDig) zuständig; hier sollen konkrete Fälle via FMHTarifdienst eingereicht werden.

Für alle übrigen Streitfragen ist die kantonale Paritätische Kommission PVK (KVG) zuständig. Das Verfahren richtet sich nach dem kantonalen PVK-Reglement. Einzelne Kantone haben gewisse Themen von der Zuständigkeit der PVK ausgenommen. Der Arzt erkundigt sich am besten bei seiner kantonalen Ärztegesellschaft.

Tarifergänzungen werden von der Paritätischen Tarifkommission (PTK) erarbeitet; danach sind sie von den vier Partnern von TARMED Suisse (FMH, H+, santésuisse und MTK) zu ratifizieren und vom Bundesrat zu genehmigen. Im Bereich SwissDRG stellen die Partner der SwissDRG AG ${ }^{125}$ Anträge für die Differenzierung bestehender oder für die Aufnahme neuer Leistungen. Auf der Basis dieser Leistungen und der Kostendaten der Spitäler nimmt die SwissDRG AG die Kalkulation vor. Ergänzungen und Änderungen der Tarifstruktur SwissDRG müssen vom Bundesrat genehmigt werden.

Sollte das Spital mit einer Kodierung des Revisors/der Revisorin nicht einverstanden und eine Bereinigung der Differenzen nicht möglich sein, so muss von beiden Parteien eine gemeinsame Anfrage beim Kodierungssekretariat des Bundesamtes für Statistik (BFS) eingereicht werden. Das BFS entscheidet innert 30 Werktagen nach Erhalt der Anfrage. Besteht die Meinungsverschiedenheit weiter, kann das

122 Art. 89 Abs. 1 KVG.

123 Im ambulanten und spitalambulanten Bereich.

124 Die qualitative Dignität gibt an, welche fachlichen Qualifikationen gemäss Weiterbildungsordnung notwendig sind, um eine bestimmte Leistung zu Lasten der Sozialversicherungen abzurechnen (Facharzttitel, Schwerpunkte, Fähigkeitsausweise). Die qualitativen Dignitäten sind bei jeder einzelnen Leistung in der TARMED-Tarifstruktur vermerkt.

$125 \mathrm{H}+, \mathrm{FMH}$, santésuisse, Gesundheitsdirektorenkonferenz GDK, Medizinaltarifkommission UVG, MV/IV (MTK). 
BFS den Fall der Schweizerischen Expertengruppe für Klassifikationen im Gesundheitswesen übertragen, die als letzte Instanz entscheidet. Die Antwort der Expertengruppe erfolgt spätestens einen Monat nach Erhalt der Anfrage.

\subsection{Behandlung von Patienten in der Unfall-, Militär- und Invalidenversicherung}

Gemäss Unfallversicherungsgesetz (UVG) ist versichert, wer Arbeitnehmer ist. Mit der Militärversicherung übernimmt der Bund die Verantwortung für die Gesundheitsschäden, die während den angeordneten Diensten eintreten. Wer AHV-Beiträge bezahlt, ist gleichzeitig auch bei der IV versichert. Ziel der Invalidenversicherung ist es, mit Eingliederungsmassnahmen Invalidität zu verhindern oder mit Geldleistungen die Existenzgrundlage zu sichern.

\section{Unfallversicherung}

Alle Arbeitnehmer müssen nach UVG versichert sein. Selbstständigerwerbende können sich freiwillig nach UVG versichern. Wer mehr als acht Stunden pro Woche arbeitet, ist auch für den Nichtbetriebsunfall - also über die Arbeitszeit hinaus - versichert. Wer nicht UVG-versichert ist und einen Unfall erleidet, ist gemäss KVG bei seiner Krankenkasse versichert. In diesem Fall muss der Arzt nach dem Taxpunktwert für Krankenkassenpatienten abrechnen.

Im Gegensatz zum KVG ist die obligatorische Unfallversicherung eine kausale Versicherung: Nur wenn die gesetzlichen Kriterien für einen Unfall, eine unfallähnliche Körperschädigung oder eine Berufskrankheit erfüllt sind, zahlt die Unfallversicherung die zweckmässige Behandlung.

Den Unfall definiert das Gesetz als «die plötzliche, nicht beabsichtigte schädigende Einwirkung eines ungewöhnlichen äusseren Faktors auf den menschlichen Körper, die eine Beeinträchtigung der körperlichen, geistigen oder psychischen Gesundheit oder den Tod zur Folge hat». ${ }^{126}$

Als unfallähnliche Körperschädigungen anerkannt - und damit auch ohne ungewöhnliche äussere Einwirkung dem Unfall rechtlich gleichgestellt - sind die folgenden Körperschädigungen, «sofern sie nicht eindeutig auf eine Erkrankung oder eine Degeneration zurückzuführen sind: a. Knochenbrüche; b. Verrenkungen von Gelenken; c. Meniskusrisse; d. Muskelrisse; e. Muskelzerrungen; f. Sehnenrisse; g. Bandläsionen; h. Trommelfellverletzungen». ${ }^{127}$

127 Art. 9 Unfallversicherungsverordnung UVV. Soll mit der laufenden UVG-Revision (Botschaft vom 29. Mai 2008) zu Art. 6 Abs. 2 UVG werden. 
Berufskrankheiten gemäss UVG sind Krankheiten, «die bei der beruflichen Tätigkeit ausschliesslich oder vorwiegend durch schädigende Stoffe oder bestimmte Arbeiten verursacht worden sind. Der Bundesrat erstellt die Liste dieser Stoffe und Arbeiten sowie der arbeitsbedingten Erkrankungen. [...] Als Berufskrankheiten gelten auch andere Krankheiten, von denen nachgewiesen wird, dass sie ausschliesslich oder stark überwiegend durch berufliche Tätigkeit verursacht worden sind.» ${ }^{128}$

Das UVG hält fest, dass der Versicherer - unter angemessener Rücksichtnahme auf den Versicherten und seine Angehörigen - die nötigen Anordnungen zur zweckmässigen Behandlung des Versicherten treffen kann. Dies bedeutet, dass die Behandlungsführung rechtlich gesehen beim UVG-Versicherer ist (sogenanntes Naturalleistungsprinzip) - und nicht bei der behandelnden Ärztin - , obschon der Unfallversicherer in der Realität nicht oft in die Behandlung eingreift.

Anders als im KVG sind die Pflichtleistungen nicht auf Verordnungsstufe näher definiert. Das Gesetz schreibt lediglich vor, dass der Versicherte Anspruch auf die zweckmässige Behandlung der Unfallfolge hat. In der Regel werden die Bestimmungen zur Krankenversicherung analog angewendet. Die Unfallversicherer übernehmen aber ihnen sinnvoll erscheinende Behandlungen traditionell auch dann, wenn diese gemäss KVG keine Pflichtleistung wären. Für den UVG-Versicherer sind Behandlungskosten eben nicht bloss Kosten, sondern eine Investition zur Vermeidung von Taggeld und Rente.

Die UVG-Versicherung umfasst auch Taggeld und Rente. Bei Selbstverschulden können diese Versicherungsleistungen, nicht aber die Behandlungskosten, gekürzt werden.

Unfallversicherte können den Arzt, den Zahnarzt, den Chiropraktor, die Apotheke und die Heilanstalt frei wählen. ${ }^{129}$ Die Zusammenarbeit zwischen Leistungserbringern und Versicherern muss gemäss UVG vertraglich geregelt werden, ${ }^{130}$ für Medizinalpersonen zudem gesamtschweizerisch. Eine Tarifgenehmigungspflicht hat der Gesetzgeber ausdrücklich nicht vorgesehen. Für ambulante ärztliche Leistungen gilt der TARMED (UV-MV-IV-Tarifvertrag zwischen der FMH und der MTK), für stationäre Spitalleistungen gilt die SwissDRG-Tarifstruktur.

Sowohl für die Streitigkeiten zwischen Patient und UVG-Versicherer als auch zwischen Leistungserbringer und UVG-Versicherer gelten dieselben Regeln wie in der Krankenversicherung (vgl. Kapitel 4.15.). Einziger Unterschied: Die Paritätische Kommission PVK ist nicht kantonal, sondern gesamtschweizerisch für alle UVsowie die MV- und IV-Fälle organisiert.

128 Art. 9 Abs. 1 und 2 UVG.

129 Art. 10 Abs. 2 UV; Um UVG-Patienten behandeln zu können, genügt die

Berufsausübungsbewilligung. Dies gilt bei Redaktionsschluss auch

für die KVG-Behandlung, doch könnte dies wieder ändern, siehe bei 3.12.

130 Art. 56 Abs. 1 UVG. 


\section{Militärversicherung}

Versichert sind nicht nur die Angehörigen der Armee und Personen, deren Beruf ein ähnliches Risiko wie der Militärdienst einschliesst, sondern auch Zivilschutzleistende, Zivildienstleistende, Teilnehmer an Veranstaltungen von Jugend+Sport und Zivilpersonen, die an Übungen der Armee und des Zivilschutzes teilnehmen. Versichert sind zudem auch Teilnehmer an friedenserhaltenden Aktionen und Guten Diensten des Bundes (Gelb- und Blaumützen) sowie Teilnehmer an Aktionen des Bundes, sofern sie Angehörige des Schweizerischen Katastrophenhilfekorps sind. Berufsmilitärs können sich nach ihrer Pensionierung freiwillig versichern.

Gemäss Militärversicherungsgesetz (MVG) erstreckt sich die Militärversicherung, die von der Suva geführt wird, auf «jede Gesundheitsschädigung, die während des Dienstes in Erscheinung tritt und gemeldet oder sonstwie festgestellt wird». ${ }^{131}$ Für die Haftung der Militärversicherung spielt es keine Rolle, ob der Gesundheitsschaden durch Unfall, Krankheit oder Suizidversuch hervorgerufen wurde.

Bei einer Gesundheitsschädigung, die während des Dienstes in Erscheinung tritt, besteht die gesetzliche Vermutung, dass das Leiden durch den Dienst verursacht wurde. Das heisst, die MV muss im Zweifelsfall beweisen, dass eine Schädigung bereits vor dem Dienst bestanden hat oder sich nicht während des Dienstes verschlechtert hat.

Für die erst nach Schluss eines Dienstes gemeldete Gesundheitsschädigung sowie für nach dem Dienst festgestellte Spätfolgen oder Rückfälle haftet dagegen die Militärversicherung nur dann, wenn mit überwiegender Wahrscheinlichkeit eine Entstehung oder eine Verschlimmerung der Schädigung während des Dienstes anzunehmen ist. Die Beweislast trägt in diesem Fall der Versicherte.

Wird anlässlich der Eintrittsmusterung eine vordienstliche Gesundheitsschädigung festgestellt, übernimmt die Militärversicherung während eines Jahres ab Dienstentlassung die volle Haftung, wenn der Versicherte trotzdem Dienst leisten muss und wenn während des Dienstes eine Verschlimmerung auftritt.

Wie im UVG gilt auch hier das sogenannte Naturalleistungsprinzip: «Die Militärversicherung sorgt für die Heilbehandlung. Sie ordnet deren Wiederaufnahme an, wenn diese medizinisch angezeigt ist oder wenn davon eine erhebliche Verbesserung der Erwerbsfähigkeit des Versicherten erwartet werden kann.» ${ }^{132}$ Die Militärversicherung zahlt im «tiers payant» ohne Franchise und Selbstbehalt. Es gilt der TARMED (UV-MV-IV-Tarifvertrag). 
Auch im MVG gelten die WZW-Kriterien. Die Behandlung ist aber nicht strikte auf die für die Krankenkassen gültigen Pflichtleistungen beschränkt. Zudem werden auch Reise-, Transport-, Such- und Rettungskosten vergütet. Die MV vergütet - ohne formelle Listenbeschränkung - einfache und zweckmässige Hilfsmittel für die Verbesserung des Gesundheitszustandes sowie für die berufliche und soziale Eingliederung.

Militärversicherte haben ein bedingt freies Wahlrecht unter Ärzten und Spitälern: Sie können zwar frei wählen, müssen aber die allfällige Tarifdifferenz selbst bezahlen, wenn sie nicht - Notfälle ausgenommen - die nächstgelegene geeignete Anstalt oder Medizinalperson wählen ${ }^{133}$ oder wenn sie sich halbprivat oder privat behandeln lassen.

Bei Streitigkeiten zwischen Versichertem und Versicherer gelten dieselben Regeln wie in der Krankenversicherung (vgl. Kap. 4.14). Für die Streitigkeiten zwischen Leistungserbringer und MVG-Versicherer gelten dieselben Regeln wie in der Unfallversicherung (siehe oben).

\section{Invalidenversicherung}

Mit der 5. Revision des Invalidenversicherungsgesetzes IVG ${ }^{134}$ haben Versicherte bis zum vollendeten 20. Altersjahr Anspruch auf medizinische Massnahmen, die nicht auf die Behandlung des Leidens an sich, sondern unmittelbar auf die Eingliederung ins Erwerbsleben oder in den Aufgabenbereich gerichtet sind. Die aus medizinischer Sicht in jedem Fall nötige Behandlung auf Kosten von Krankenkasse oder Unfallversicherung muss abgeschlossen sein.

Für medizinische Behandlungen gemäss IVG muss vorgängig eine Kostengutsprache eingeholt werden. Wie im UVG gelten die WZW-Kriterien, und formal sind die Behandlungen nicht auf die KVG-Pflichtleistungen beschränkt. Die Versicherten können unter den geeigneten Medizinalpersonen frei wählen. Es gilt der TARMED (UV-MV-IV-Tarifvertrag).

Bei den über 20-Jährigen muss die Krankenkasse bzw. die Unfallversicherung die Kosten für die medizinischen Massnahmen übernehmen. Wer durch Krankheit invalid wird, muss für Eingliederungsmassnahmen Franchise und Selbstbehalt bezahlen. Die IV übernimmt weiterhin und auch für erwachsene Versicherte jene Hilfsmittel für die Eingliederung, «deren er für die Ausübung der Erwerbstätigkeit oder der Tätigkeit im Aufgabenbereich, zur Erhaltung oder Verbesserung der Erwerbsfähigkeit, für die Schulung, die Aus- und Weiterbildung oder zum Zwecke der funktionellen Angewöhnung bedarf». ${ }^{135}$ 
Am 1.Januar 2012 ist der erste Teil der 6. IV-Revision (Massnahmepaket 6a) in Kraft getreten. Ein Hauptbestandteil der Revision ist die eingliederungsorientierte Rentenrevision nach dem Motto «Eingliederung vor Rente». Zur Verbesserung der Erwerbsfähigkeit der Rentner können jederzeit Massnahmen zur Wiedereingliederung umgesetzt werden. Neben den bestehenden Massnahmen sind neu Beratung und Begleitung für die Versicherten und deren Arbeitgeber vorgesehen. Ziel dabei ist der Erhalt des Arbeitsplatzes. Weiter werden Renten, die bei pathogenetisch-ätiologisch unklaren syndromalen Beschwerdebildern ohne nachweisbare organische Grundlage gesprochen wurden, innerhalb von drei Jahren ab dem 1. Januar 2012 überprüft. ${ }^{136}$ Es geht dabei konkret um die Frage, ob eine Erwerbstätigkeit trotz vorhandener Beschwerden aus objektiver Sicht zumutbar ist. Von der Überprüfung ausgenommen sind Personen ab 55 Jahren und solche, die seit mehr als 15 Jahren eine Rente beziehen. Für beide gilt die Besitzstandsgarantie.

Neu wurde auch der Assistenzbeitrag für Menschen mit Behinderung eingeführt. Er ergänzt die Hilflosenentschädigung, den Intensivpflegezuschlag, die Spitexleistungen im Bereich Grundpflege sowie die Dienstleistungen Dritter anstelle eines Hilfsmittels. Anspruch auf einen Assistenzbeitrag haben grundsätzlich handlungsfähige Volljährige, die eine Hilfslosenentschädigung beziehen und zu Hause leben oder zu Hause leben möchten. Zudem ist für die Auszahlung ein Arbeitsvertrag mit einer Assistenzperson erforderlich, wobei nur natürliche Personen als Assistenzpersonen zugelassen sind, die nicht in gerader Linie mit der versicherten Person verwandt sind. Weiter haben Anspruch auf einen Assistenzbeitrag, sofern die Voraussetzungen gegeben sind, Minderjährige sowie Volljährige mit eingeschränkter Handlungsfähigkeit. ${ }^{137}$

Im IVG gilt seit Juli 2006 wieder das Vorbescheidsverfahren: In einem Vorbescheid teilt die IV-Stelle der versicherten Person den vorgesehenen Endentscheid über ein Leistungsbegehren oder den Entzug oder die Herabsetzung einer bisher gewährten Leistung mit. Ist vom vorgesehenen Entscheid die Leistungspflicht eines anderen Versicherungsträgers betroffen, so muss die IV-Stelle diesen vor Erlass der Verfügung anhören.

Erst nach dem Vorbescheid kann der Versicherte eine Verfügung verlangen. Ist er mit dieser nicht einverstanden, führt der Weg ans kantonale Versicherungsgericht und gegebenenfalls ans Bundesgericht (EVG). Wird das kantonale Versicherungsgericht angerufen, gelten von diesem Zeitpunkt an dieselben Bestimmungen wie im KVG (vgl. Kap. 4.14.). Allerdings sind Prozesse gemäss IVG nicht mehr unentgeltlich. Bei Streitigkeiten zwischen Leistungserbringer und IV-Stelle gelten dieselben Regeln wie in der Unfallversicherung. 


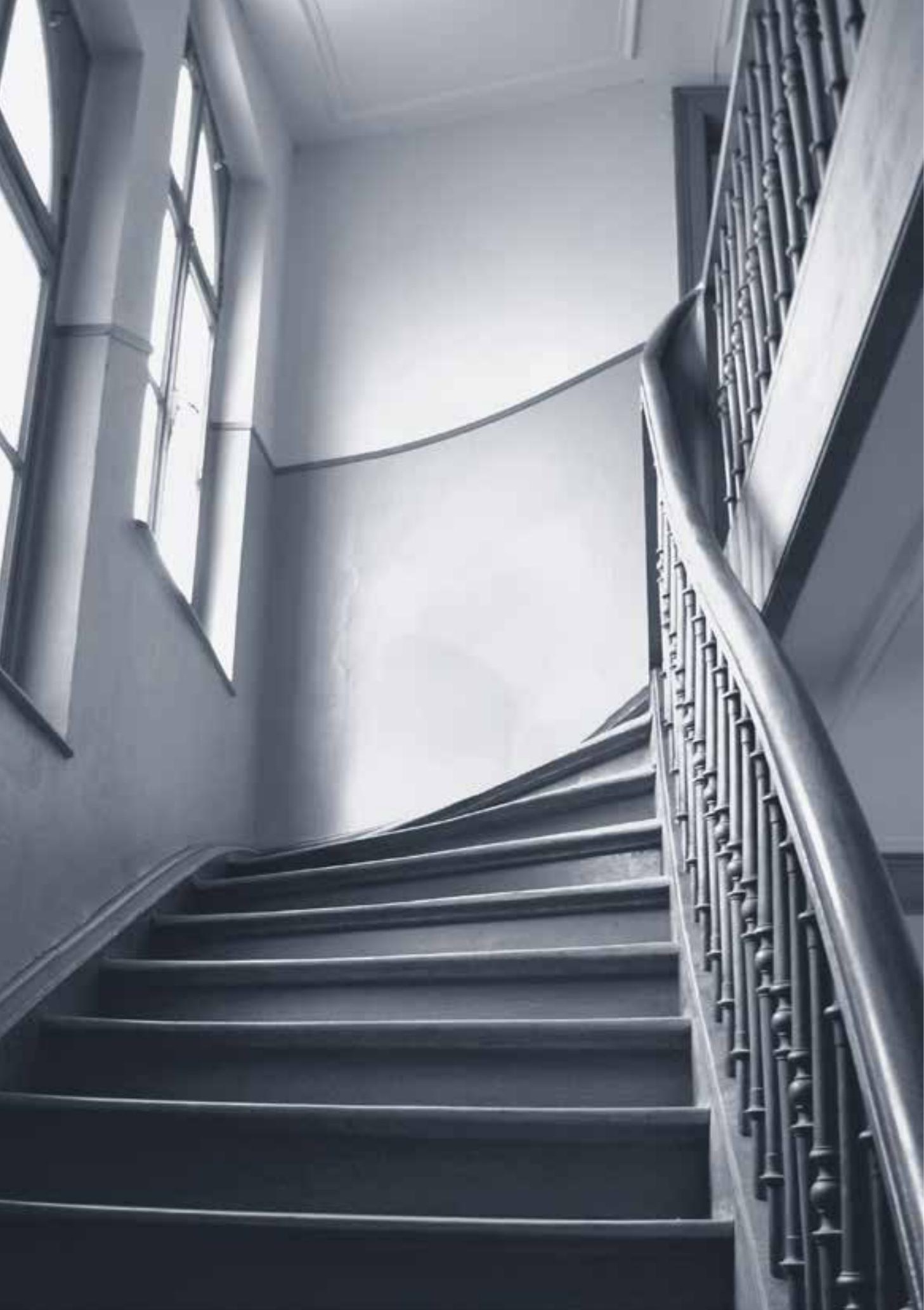




\section{Spezielle Situationen bei der Behandlung von Patientinnen und Patienten}

\subsection{Genetische Untersuchungen}

\section{Seit dem Jahr 2007 sind genetische Untersuchungen in einem speziellen Gesetz geregelt. Dieses Gesetz schützt einerseits die Rechte der betroffenen Personen, soll aber auch die Qualität der Untersuchungen sichern. ${ }^{138}$}

Das Bundesgesetz über genetische Untersuchungen am Menschen (GUMG) erfasst sämtliche Untersuchungen, die unmittelbar die Abklärung ererbter oder während der Embryonalphase erworbener Eigenschaften des Erbgutes bezwecken. Die gesetzliche Regelung gilt dabei unabhängig von der gewählten Methode, sodass z.B. auch bildgebende Verfahren zur pränatalen Risikoabklärung darunter fallen, sofern sie dem genannten Zweck dienen. Hingegen werden zu Forschungszwecken durchgeführte Untersuchungen nicht erfasst.

Das Gesetz gewährleistet einerseits den Schutz bzw. die Rechte der betroffenen Personen (Diskriminierungsverbot, Informations- und Einwilligungsrechte, Recht auf Nichtwissen), enthält aber auch Vorgaben mit dem Ziel der Qualitätssicherung. So ist die Veranlassung genetischer Untersuchungen Ärztinnen und Ärzten vorbehalten, wobei diese für präsymptomatische und pränatale Untersuchungen über eine entsprechende Weiterbildung verfügen müssen. Pränatale und Reihenuntersuchungen unterliegen zusätzlichen spezifischen Voraussetzungen.

Weiter benötigen die Laboratorien, die zyto- und molekulargenetische Untersuchungen durchführen, eine Bewilligung des Bundesamtes für Gesundheit. Schliesslich dürfen genetische In-vitro-Diagnostika nur an medizinische Fachpersonen oder den Fachhandel (Grossisten), nicht aber an Private abgegeben werden.

Neben den Untersuchungen im medizinischen Bereich regelt das Gesetz u.a. auch die Erstellung von DNA-Profilen zur Klärung der Abstammung. Um Interessenkollisionen zu vermeiden, darf ein urteilsunfähiges Kind nicht von derjenigen

138 Siehe u.a. Peter Miny, Siv Fokstuen, Praktische Konsequenzen für Anbieter, Ärzte und Patienten, SÄZ 13/2007; 580- 82; Cristina Benedetti, BAG: Genetische Untersuchungen beim Menschen ab dem 1. April 2007 gesetzlich geregelt, SÄZ 13/2007; 583-85; sowie Peter Suter, Bundesgesetz über genetische Untersuchungen beim Menschen (GUMG), SÄZ 13/2007; 586f.; SAMW, Genetische Untersuchungen im medizinischen Alltag - Ein Leifaden für die Praxis, 2. Auflage 2011. 
Person vertreten werden, von der die Abstammung geklärt werden soll. Hier ist die Kindes- und Erwachsenenschutzbehörde beizuziehen. Bei vorgeburtlichen Vaterschaftsabklärungen bestehen zusätzliche Informationspflichten.

Das GUMG regelt zudem die genetischen Untersuchungen im Versicherungsbereich und begrenzt die Rechte der Versicherer, das heisst, Versicherer dürfen keine genetischen Untersuchungen durchführen lassen. Sie dürfen in den folgenden Fällen zudem nicht nach früheren Untersuchungsergebnissen fragen:

- Sozialversicherer (Pensionskassen dürfen auch für den überobligatorischen Bereich nicht nach allfälligen früheren Testergebnissen fragen);

- Taggeldversicherer;

- Lebensversicherer bei einer Versicherungssumme von höchstens 400000 Franken;

- freiwillige Invaliditätsversicherungen mit einer Jahresrente von höchstens 40000 Franken.

Die Nachforschung seitens des Versicherers nach Ergebnissen von früheren genetischen Untersuchungen ist nur dann zulässig,

- wenn ein Versicherungsvertrag erstens nicht der soeben erwähnten Regel untersteht (konkret v.a.: Lebensversicherungen über 400000 Franken, Zusatzversicherungen zur Krankenversicherung und Invalidenversicherung über 40000 Franken), und

- wenn die betreffende Untersuchung technisch und in der medizinischen Praxis zuverlässige Ergebnisse liefert und der wissenschaftliche Wert der Untersuchung für die Prämienberechnung nachgewiesen ist.

Der Versicherer hat zudem nicht bei der antragstellenden Person, sondern bei der beauftragten Ärztin bzw. beim beauftragten Arzt nachzufragen. Letztere teilen dem Versicherer zudem lediglich mit, in welcher Risikokategorie die betreffende Person einzuteilen ist.

Schliesslich regelt das Gesetz die Zulässigkeit genetischer Untersuchungen im Haftpflichtbereich (d.h. im Kontext der Schadensberechnung und der Schadenersatzbemessung) und im Arbeitsbereich: Von wenigen Ausnahmen abgesehen dürfen im Zusammenhang mit der Arbeit keine genetischen Untersuchungen durchgeführt werden. 


\subsection{Fortpflanzungsmedizin}

Das Fortpflanzungsmedizingesetz legt fest, unter welchen Voraussetzungen die Verfahren der medizinisch unterstützten Fortpflanzung beim Menschen angewandt werden dürfen. Es schützt die Menschenwürde, die Persönlichkeit sowie die Familie und verbietet missbräuchliche Anwendungen der Bio- und der Gentechnologie.

Nach dem Bundesgesetz über die medizinisch unterstützte Fortpflanzung dürfen Verfahren wie die künstliche Insemination oder die In-vitro-Fertilisation mit Embryotransfer erstens nur bei verschiedengeschlechtlichen Paaren und zweitens nur zur Überwindung von Unfruchtbarkeit oder zur Vermeidung der Übertragung einer schweren, unheilbaren Krankheit angewendet werden. Das Geschlecht oder bestimmte andere Eigenschaften des Kindes dürfen dabei nicht beeinflusst werden, ausser es ist notwendig, um die Übertragung einer schweren, unheilbaren Krankheit zu vermeiden. Die Präimplantationsdiagnostik ist (noch) verboten.

Während die Verwendung gespendeter Samenzellen zulässig ist, sind die Eizellen- und Embryonenspende sowie die Leihmutterschaft generell verboten. Keimzellen und imprägnierte Eizellen ${ }^{139}$ dürfen maximal fünf Jahre konserviert werden, Embryonen ${ }^{140}$ hingegen nicht. Nach dem Gesetz dürfen zudem pro Behandlungszyklus der Frau nur so viele imprägnierte Eizellen zu Embryonen entwickelt werden, als zur Herbeiführung einer Schwangerschaft erforderlich sind, höchstens aber drei.

Selbstverständlich dürfen Verfahren der Fortpflanzungsmedizin nur angewandt werden, wenn eine schriftliche Einwilligung des umfassend aufgeklärten Paares vorliegt; dies gilt auch für die künstliche Insemination.

Die Anwendung von Fortpflanzungsmedizinverfahren, die Konservierung von Keimzellen und imprägnierten Eizellen sowie die Vermittlung von Samenzellen bedürfen einer Bewilligung des jeweiligen Kantons. ${ }^{141}$

Das Fortpflanzungsmedizingesetz wird derzeit überarbeitet. Im Wesentlichen soll das Verbot der Präimplantationsdiagnostik durch eine Zulassung unter strengen Voraussetzungen ersetzt werden. Die Gesetzesänderung wird nicht vor 2015 in Kraft treten.

139 Imprägniert ist eine Eizelle, wenn sie zwar befruchtet ist, die Kernverschmelzung aber noch nicht stattgefunden hat.

140 Der Embryo wird gesetzlich als die Frucht von der Kernverschmelzung bis zum Abschluss der Organentwicklung definiert.

141 Keine Bewilligung ist nötig für die künstliche Insemination mit Samenzellen des Partners. 


\subsection{Schwangerschaftsabbruch}

Der Schwangerschaftsabbruch ist dann erlaubt und bleibt straflos, wenn er innerhalb der ersten 12 Wochen oder aufgrund medizinischer Indikation erfolgt.

Seit 2002 ist die sogenannte Fristenlösung in Kraft. Der Abbruch einer Schwangerschaft ist straflos, «wenn er innerhalb von zwölf Wochen seit Beginn der letzten Periode auf schriftliches Verlangen der schwangeren Frau, die geltend macht, sie befinde sich in einer Notlage, durch eine zur Berufsausübung zugelassene Ärztin oder einen zur Berufsausübung zugelassenen Arzt vorgenommen wird. Die Ärztin oder der Arzt hat persönlich mit der Frau vorher ein eingehendes Gespräch zu führen und sie zu beraten.» ${ }^{142}$ Ein späterer Schwangerschaftsabbruch ist bei medizinischer Indikation ebenfalls straflos. Dabei gilt: «Die Gefahr [für die Frau] muss umso grösser sein, je fortgeschrittener die Schwangerschaft ist.»

Eine urteilsfähige jugendliche schwangere Frau kann den Behandlungsvertrag ohne Zustimmung der Eltern abschliessen. ${ }^{143}$

Die Kantone führen Listen der Praxen und Spitäler, die einen straflosen Schwangerschaftsabbruch und die Beratung der Frau durchführen dürfen. Der Arzt oder die Ärztin muss den Abbruch zu statistischen Zwecken der zuständigen Gesundheitsbehörde des Kantons melden (vgl. Kap. 7.3). Diese Meldung erfolgt anonym.

\subsection{Sterilisation}

Seit dem Jahr 2005 regelt ein Bundesgesetz die Sterilisation. Durch eine Sterilisation wird die Fortpflanzungsfähigkeit einer Person auf Dauer aufgehoben. Nicht als Sterilisation gelten hingegen Heileingriffe, deren unvermeidliche Begleiterscheinung die Aufhebung der Fortpflanzungsfähigkeit ist.

Urteilsfähige Erwachsene dürfen sterilisiert werden, wenn sie über den Eingriff umfassend informiert wurden und diesem frei und schriftlich zugestimmt haben. «Wer den Eingriff durchführt, muss in der Krankengeschichte festhalten, auf Grund welcher Feststellungen er auf die Urteilsfähigkeit der betroffenen Person geschlossen hat.» ${ }^{144}$

Personen unter umfassender Beistandschaft, die urteilsfähig sind, müssen ebenfalls umfassend aufgeklärt werden und selbst schriftlich und frei zustimmen; bei ihnen müssen aber zusätzlich der gesetzliche Vertreter und die Erwachsenenschutzbehörde zustimmen. Die Erwachsenenschutzbehörde muss zudem eine ärztliche Zweitmeinung und nötigenfalls ein psychiatrisches Gutachten zur Urteilsfähigkeit einholen.

143 Vgl. Kapitel 3.1.: Die Behandlung ist kassenpflichtig, deshalb müssen die Eltern nicht zustimmen. 144 Art. 5 Sterilisationsgesetz. 
Die Sterilisation einer Person unter 18 Jahren ist grundsätzlich verboten. Nur unter strengen Voraussetzungen ist eine Ausnahme bei einer über 16-jährigen, dauernd urteilsunfähigen Person möglich. Die betroffene Person, eine ihr nahestehende Person oder ihr Beistand können zudem den Entscheid der Erwachsenenschutzbehörde innert 30 Tagen anfechten.

\subsection{Transplantationsmedizin}

Das Transplantationsgesetz legt fest, unter welchen Voraussetzungen Organe, Gewebe oder Zellen zu Transplantationszwecken verwendet werden dürfen. Es soll einerseits dazu beitragen, dass menschliche Organe, Gewebe oder Zellen für Transplantationszwecke zur Verfügung stehen. Es soll aber auch den missbräuchlichen Umgang mit Organen, Gewebe oder Zellen verhindern und die Menschenwürde, die Persönlichkeit und die Gesundheit schützen.

Bei einer verstorbenen Person ist die Entnahme von Organen zulässig, wenn sie vor ihrem Tod zugestimmt hat und der Tod nach den entsprechenden Richtlinien der SAMW festgestellt worden ist. Liegt seitens der verstorbenen Person keine dokumentierte Erklärung vor (z. B. Spenderausweis, Patientenverfügung) und ist eine solche auch nicht bekannt, können die nächsten Angehörigen der Entnahme zustimmen. Diese haben bei ihrer Entscheidung aber den mutmasslichen Willen der verstorbenen Person zu beachten. Ohne eine explizite Zustimmung der verstorbenen Person oder der Angehörigen ist die Entnahme unzulässig. Hat die verstorbene Person die Entscheidbefugnis nachweislich einer Vertrauensperson übertragen, tritt diese an die Stelle der Angehörigen.

Das Gesetz enthält eine vergleichsweise liberale Regelung zur Lebendspende durch urteilsfähige und mündige Personen: Diese müssen schriftlich und nach umfassender Aufklärung zugestimmt haben, und deren Leben oder Gesundheit darf nicht ernsthaft gefährdet sein. Zudem darf für die empfangende Person keine andere Therapie mit vergleichbarem Nutzen bestehen. Eine Spende kann sowohl zugunsten einer bekannten als auch einer unbekannten Person erfolgen, eine persönliche Beziehung ist nicht notwendig. Die Lebendspende urteilsunfähiger oder minderjähriger Personen ist demgegenüber nur ausnahmsweise für die Entnahme regenerierbarer Gewebe und Zellen zulässig. 
Der behandelnde Arzt ist verpflichtet, Patienten, bei denen eine Organtransplantation indiziert ist, mit deren Einwilligung unverzüglich einem Transplantationszentrum zu melden. Die erforderlichen Daten werden in der Folge auf einer gesamtschweizerischen, von der nationalen Zuteilungsstelle ${ }^{145}$ geführten Warteliste aufgenommen. Die Zuteilung der verfügbaren Organe erfolgt nach gesetzlich definierten Kriterien zentral und patientenbezogen durch die nationale Zuteilungsstelle. Das Transplantationsgesetz legt zudem verschiedene Bewilligungs- und Meldepflichten fest. ${ }^{146}$

Diskutiert wird aktuell eine Teilrevision des Transplantationsgesetzes. Neu sollen Grenzgängerinnen und Grenzgänger, die in der Schweiz krankenversichert sind, bei der Zuteilung von Organen den Personen mit Wohnsitz in der Schweiz gleichgestellt werden. Gleichzeitig wird eine Änderung von Bestimmungen vorgeschlagen, die in der praktischen Umsetzung zu Unsicherheiten geführt haben. Diese betreffen einerseits den Zeitpunkt der Anfrage an die Angehörigen für eine Organspende sowie andererseits die Vertretungsbefugnis bei der Zustimmung zu vorbereitenden medizinischen Massnahmen. Schliesslich werden Bestimmungen zur weiteren finanziellen Absicherung der Lebendspenderinnen vorgeschlagen.

\section{6. Ärztliche Betreuung von Sportlern; Doping}

Auch wenn der Arzt im Auftrag eines Sportverbandes tätig wird, soll er die Interessen des Sportlers bzw. des Patienten wahren. Doping ist verboten.

Wer Sportler und Sportlerinnen betreut, ist häufig auch im Auftrag eines Sportverbandes tätig. Diese Dreiecksbeziehung kann Interessenkonflikte mit sich bringen. Die FMH-Standesordnung hält dazu fest: «Von Notfällen abgesehen enthalten sich Ärztinnen und Ärzte im Auftrag von Sportverbänden [...] in dieser Funktion jeder Tätigkeit, die über ihre spezifischen Aufgaben hinausführt. [...] Im Auftrag von Sportverbänden tätige Ärzte und Ärztinnen sind sich des Interessenkonfliktes bewusst, welcher zwischen der untersuchten Person einerseits und dem Auftraggeber [...] anderseits entstehen kann [...]. Bei der Weiterleitung von Informationen bemühen sich Arzt und Ärztin, die Interessen beider Parteien angemessen zu berücksichtigen.» ${ }^{147}$

145 Die nationale Zuteilungsstelle wird im Auftrag des Bundes von der Stiftung Swisstransplant geführt.

146 Eine umfassende Übersicht über die gesetzlichen Rahmenbedingungen ermöglicht die Website des Bundesamtes für Gesundheit (www.bag.admin.ch/transplantation/index.html?lang=de). Eine Einführung für Ärzte gibt der Artikel von Conrad E. Müller, Erläuterungen zum neuen Transplantationsgesetz und dessen Umsetzung, SÄZ 30/2007; 1256 - 58.

147 Art. 27 und Art. 33 FMH-Standesordnung. Vgl. auch die Richtlinie der Ärztekammer «für die ärztliche Betreuung von Sportlern und Sportlerinnen», Anhang 5 zur FMH-Standesordnung. 
Im Zentrum der öffentlichen Diskussionen rund um die Beziehung zwischen Ärztin und Sportler steht aber weniger das Verhältnis zum Sportverband als vielmehr das Doping. Das Bundesgesetz über Turnen und Sport stellt die Mithilfe eines Arztes oder einer Ärztin bei Doping unter Strafe. Auf www.dopinginfo.ch werden jeweils die auf der Dopingliste stehenden Medikamente oder anderen Substanzen aufgeführt.

Doping ist verboten, wenn der Athlet an einem Anlass des «reglementierten Wettkampfsports» teilnimmt. Dazu gehören Sportanlässe, die von einem bei Swiss Olympic angeschlossenen Verband oder Verein oder deren internationalen Dachverbänden organisiert werden, sowie das Training vor und die Regeneration nach dem Wettkampf. Wer an solchen Anlässen teilnimmt, gilt als Athletin und nicht als normale Patientin.

Falls der Arzt bei einer Sportlerin die Verabreichung eines Medikamentes mit verbotenen Substanzen aus medizinischen Gründen als notwendig erachtet, hat er vorgängig die Einwilligung der Vertrauensärztin der Fachkommission für Dopingbekämpfung (FDB) von Swiss Olympic einzuholen.

\subsection{Behandlung und Betreuung von älteren, pflegebedürftigen Menschen}

\section{Bei der Betreuung von älteren Menschen in Heimen besteht ein Konflikt zwischen Hilfsbedürftigkeit und Autonomie. Der Schutz ihrer Persönlichkeit und ihrer Würde hat einen hohen Stellenwert.}

Der rechtliche und psychosoziale Schutz von urteilsunfähigen Personen, die in Wohn- und Pflegeeinrichtungen leben, wurde durch das neue Erwachsenenschutzrecht verbessert. ${ }^{148}$ Wird eine urteilsunfähige Person für längere Dauer in einem Alters- oder Pflegeheim betreut, so muss zwingend ein schriftlicher Betreuungsvertrag abgeschlossen werden, der Auskunft über die Leistungen der Einrichtung und das geschuldete Entgelt gibt. Es ist Sache der vertretungsberechtigten Personen, den Vertrag für die urteilsunfähige Person zu unterzeichnen (mehr dazu siehe Kap. 3.).

Der Autonomie der betagten Menschen kommt eine grosse Bedeutung zu. Die freie Arztwahl ist deshalb auch im Heim gewährleistet, soweit nicht wichtige Gründe dagegen sprechen (mehr dazu siehe Kap. 3.). Die Patientin und ihre Angehörigen sollen jedenfalls wissen, welche Ärztin zuständig ist. Die entsprechende SAMW-Richtlinie ${ }^{149}$ hält fest, dass für die adäquate Betreuung ein persönlicher Kontakt zwischen dem Arzt und der älteren, pfegebedürftigen Person unabding- 
bar ist. Im Heim ist eine umfassende Beurteilung der Situation der Patienten besonders wichtig: So sollen die behandelnde Ärztin, das Pflegepersonal und die Therapeuten ihre Enscheide auf die gemeinsame Evaluation medizinischer, psychischer, sozialer und funktionaler Aspekte und des Umfelds stützen.

Es gibt Situationen, in denen sich verwirrte, urteilsunfähige Personen nicht mehr orientieren können und sich dadurch gefährden. Das Bundesrecht regelt, unter welchen Bedingungen eine Einschränkung der Bewegungsfreiheit zulässig ist. ${ }^{150}$ Eine solche Massnahme kann angeordnet werden, um entweder eine ernsthafte Gefahr für das Leben oder die körperliche Integrität der betroffenen Person oder Dritter abzuwenden oder eine schwerwiegende Störung des Gemeinschaftslebens zu beseitigen. Die Einschränkung ist so bald als möglich wieder aufzuheben und muss protokolliert werden.

Zum Schutze der Persönlichkeit gehört es ferner, dass sich die Einrichtung um das Wohl der urteilsunfähigen Bewohner kümmert, deren Bedürfnissen Rechnung trägt und Leiden lindert. Um die Einsamkeit zu verringern, sollen Kontakte zu Personen ausserhalb der Einrichtung gefördert werden. Gemäss SAMW-Richtlinien machen Ärzte und Pflegende ältere Personen frühzeitig auf die Möglichkeit der Bezeichnung einer Vertrauensperson aufmerksam. Kümmert sich niemand von ausserhalb der Einrichtung um die betagte Person, so ist die Erwachsenenschutzbehörde zu benachrichtigen.

\subsection{Medizinische Entscheidungen am Lebensende}

\section{Medizinische Entscheidungen am Lebensende können mit einem lebensverkürzenden Effekt verbunden sein. Die klinischen Situationen in diesem Zusammenhang sind vielfältig und fast immer komplex.}

Unter medizinischen Entscheidungen am Lebensende versteht man sämtliche Handlungen und Unterlassungen, die möglicherweise oder sicher die Lebensspanne eines schwerstkranken Menschen verkürzen bzw. dessen Tod herbeiführen. Häufig spricht man in diesem Zusammenhang von «Sterbehilfe», wobei in den letzten Jahren eine Tendenz zu beobachten war, diesen Begriff zunehmend in eingeengter Bedeutung als Überbegriff für Suizidbeihilfe und aktive Sterbehilfe auf Verlangen zu verwenden. Aus Sicht der FMH ist es hingegen wichtig, zwischen Suizidbeihilfe und Sterbehilfe klar zu unterscheiden (vgl. Kap. 5.9.). Einen Überblick über die verschiedenen Formen der medizinischen Entscheidungen am Lebensende gibt die nachstehende Tabelle. 


\section{Formen medizinischer Entscheidungen am Lebensende und deren rechtliche Einordnung}

\begin{tabular}{|c|c|c|}
\hline $\begin{array}{l}\text { Medizinische Entscheidungen am } \\
\text { Lebensende (Sterbehilfe') }\end{array}$ & Todesart & Rechtlicher Status \\
\hline $\begin{array}{l}\text { Behandlungsverzicht und -abbruch in } \\
\text { Übereinstimmung mit dem } \\
\text { (mutmasslichen) Willen des Patienten } \\
\text { (passive Sterbehilfe) }\end{array}$ & natürlicher Todesfall & legal \\
\hline $\begin{array}{l}\text { Behandlung von Schmerz und anderen } \\
\text { Symptomen unter Inkaufnahme } \\
\text { einer Lebensverkürzung (indirekte } \\
\text { aktive Sterbehilfe) }\end{array}$ & natürlicher Todesfall & legal \\
\hline Beihilfe zum Suizid² & nichtnatürlicher Todesfall & $\begin{array}{l}\text { legal, falls keine eigen- } \\
\text { nützigen Motive des } \\
\text { Sterbehelfers: Art. } 115 \mathrm{StGB}\end{array}$ \\
\hline Aktive Sterbehilfe auf Verlangen ${ }^{2}$ & nichtnatürlicher Todesfall & illegal: Art. 114 StGB \\
\hline $\begin{array}{l}\text { Aktive Lebensbeendigung ohne } \\
\text { ausdrückliches Verlangen }\end{array}$ & nichtnatürlicher Todesfall & $\begin{array}{l}\text { illegal, z.B. Art. } 111 \text { StGB } \\
\text { und } 113 \mathrm{StGB}\end{array}$ \\
\hline
\end{tabular}

Sterbehilfe im weiten Sinne.

2 Beihilfe zum Suizid und aktive Sterbehilfe auf Verlangen werden zunehmend als Sterbehilfe (im engen Sinne) bezeichnet.

Was die rechtliche Einordnung dieser Entscheidungen betrifft, so gilt, dass die gezielte Tötung eines Patienten durch den Arzt in jedem Fall illegal ist, dies auch dann, wenn eine urteilsfähige Patientin ihre Tötung ausdrücklich verlangt hat. Dagegen ist die ärztliche Beihilfe zum Suizid grundsätzlich straflos (vgl. Kap. 5.9.).

Auf eine lebenserhaltende Behandlung zu verzichten oder diese abzubrechen (passive Sterbehilfe) ist erlaubt oder sogar geboten, wenn die Entscheidung dem ausdrücklichen Willen des urteilsfähigen Patienten entspricht. Ist der Patient urteilsunfähig, ist die in einer Patientenverfügung festgehaltene Anordnung massgeblich oder der Entscheid liegt bei der vertretungsberechtigten Person (vgl. Kap. 4.3.). Auch bei der sogenannten «indirekten aktiven Sterbehilfe» ist die ausdrückliche Zustimmung des urteilsfähigen Patienten bzw. beim urteilsunfähigen Patienten der Inhalt der Patientenverfügung oder der Entscheid des Vertretungsberechtigten verbindlich. Es ist allerdings umstritten, wieweit eine korrekte Schmerz- und Symptomtherapie am Lebensende überhaupt mit dem durch den Begriff «indirekte aktive Sterbehilfe» postulierten lebensverkürzenden Effekt verbunden ist. 


\section{Einige Sondersituationen}

Folgende Situationen sind in der Praxis geläufig, aber im Gesetz bisher nicht ausdrücklich geregelt:

- Nahrungs- und Flüssigkeitszufuhr bei Patientinnen im Wachkoma bzw. mit persistierendem vegetativem Status (PVS): Ohne gegenteilige Anordnung in einer Patientenverfügung ist gemäss SAMW-Richtlinien ein klinisch stabiler Patient adäquat zu ernähren und mit Flüssigkeit zu versorgen. Beim Auftreten von Komplikationen (z.B. einer Lungenentzündung) ist der Verzicht auf lebensverlängernde Massnahmen gerechtfertigt, wenn dies dem in einer Patientenverfügung niedergelegten Willen des Patienten entspricht oder die vertretungsberechtigte Person ihr Einverständnis dazu gibt.

- Ein dementer Patient verweigert zunehmend die Nahrungs-, evtl. auch die Flüssigkeitsaufnahme: Gemäss SAMW-Richtlinien ist, nach zumutbarer Diagnostik zum Ausschluss einer behandelbaren Störung (Schluckstörung, gastrointestinale Pathologie), ein solches Verhalten als verbindliche Willensäusserung zu respektieren und auf das Legen einer PEG-Sonde zur Zwangsernährung zu verzichten.

- Versetzen einer terminalen Patientin in ein kontinuierliches, tiefes Koma bis zum Tode (palliative Sedierung): Dies ist gemäss SAMW-Richtlinien erlaubt, wenn eine Symptomkontrolle nicht anders erzielt werden kann und diese Massnahme mit dem Patienten besprochen oder in einer Patientenverfügung gewünscht wurde. Eine Patientin darf aber nicht in ein kontinuierliches Koma versetzt werden, um damit gewollt den Tod herbeizuführen. Dann nämlich würde der Bereich der indirekten Sterbehilfe verlassen und eine aktive, strafbare Fremdtötung vorliegen.

- Sedierung eines zerebral schwerst und irreversibel geschädigten Patienten mit noch nicht vollständig erloschenem eigenem Atemantrieb vor Extubation: Gemäss SAMW-Richtlinien ${ }^{151}$ ist dies dann erlaubt, wenn der Tod der Patientin absehbar ist.

- Verzicht auf bzw. Einstellen einer Reanimation bei Herzkreislaufstillstand (do not resuscitate order): Es ist umstritten, inwieweit der Entscheid, nicht zu reanimieren, mit dem Patienten und/oder der vertretungsberechtigten Person abgesprochen werden muss. Die SAMW hat hierzu Richtlinien ausgearbeitet. ${ }^{152}$ Ein Verzicht auf den «informed consent» könnte rechtlich insbesondere dann begründet werden, wenn die Wahrscheinlichkeit einer erfolgreichen Reanimation verschwindend klein erscheint (futile treatment). 


\subsection{Beihilfe zum Suizid}

\section{Beihilfe zum Suizid ist in der Schweiz nicht strafbar, wenn ein urteilsfähiger Sterbewilliger den Suizid selbst begeht und wenn die helfende Person nicht selbstsüchtig handelt.}

In der Schweiz ist Beihilfe zum Suizid gemäss Artikel 115 des Strafgesetzbuchs nicht strafbar, solange sie ohne selbstsüchtige Motive erfolgt. Die Grenzen regelt das Strafgesetzbuch: «Wer aus selbstsüchtigen Beweggründen jemanden zum Selbstmord verleitet oder ihm dazu Hilfe leistet, wird, wenn der Selbstmord ausgeführt oder versucht wurde, mit Freiheitsstrafe bis zu 5 Jahren oder Geldstrafe bestraft.» Das bedeutet, dass Beihilfe zum Suizid nicht strafbar ist, wenn alle folgenden drei Bedingungen gleichzeitig erfüllt sind:

- Der Sterbewillige hatte selbst die Tatherrschaft über die zum Tode führende Handlung inne.

- Bei der beim Suizid assistierenden Person liegen keine selbstsüchtigen Beweggründe vor.

- Die Person, welche die Selbsttötung beging, war bezüglich des eigenen Sterbewunsches urteilsfähig.

Vor diesem liberalen strafrechtlichen Hintergrund hat die Schweiz in den letzten zwanzig Jahren einen Weg beschritten, der im internationalen Vergleich gegenüber der Suizidbeihilfe sehr tolerant ist.

Durch diese Entwicklung ist die traditionelle standesrechtliche Position, dass «Beihilfe zum Suizid kein Teil der ärztlichen Tätigkeit» sei, etwas aufgeweicht worden. Es wird in der Schweiz heute auch in der Ärzteschaft zunehmend anerkannt, dass Suizidbeihilfe ohne ärztliche Expertise betreffend Diagnose, Prognose sowie Möglichkeiten der Behandlung oder Symptomlinderung nicht befriedigend reguliert werden kann. Gleichzeitig darf aber nicht ausser Acht geraten, dass es hier um höchstpersönliche bzw. gesellschaftliche Fragen geht, die den Rahmen medizinischer Expertise sprengen.

Das zur Suizidbeihilfe verwendete Natrium-Pentobarbital untersteht sowohl der Betäubungsmittelverzeichnisverordnung als auch dem Heilmittelgesetz (Abgabekategorie B). Es darf also nur von Ärzten verschrieben werden, die zur selbstständigen Berufsausübung zugelassen sind, wobei diese dabei die anerkannten Regeln der medizinischen Wissenschaften zu beachten haben. Die Einhaltung der entsprechenden Pflichten wird von den kantonalen Aufsichtsbehörden überwacht; bei Pflichtverletzungen können die Kantone Disziplinarmassnahmen wie einen Verweis oder einen teilweisen bzw. vollständigen Entzug der Praxisbewilligung verfügen. 
Zur Frage, was unter den anerkannten Regeln der medizinischen Wissenschaften im Kontext der Rezeptierung von Natrium-Pentobarbital zum Zweck der Suizidbeihilfe zu verstehen ist, hat das Bundesgericht 2006 festgehalten, dass die Rezeptierung von Natrium-Pentobarbital mit dem Ziel, einem Patienten den Suizid zu ermöglichen, eine «den Regeln der ärztlichen Berufs- und Sorgfaltspflichten entsprechend vorgenommene Diagnose, Indikationsstellung und ein Aufklärungsgespräch» verlangt. Des Weiteren ist die Prüfung und Dokumentation der Urteilsfähigkeit des Patienten für seinen Sterbewunsch zentral. Die Beurteilung der Urteilsfähigkeit kann dabei letztlich nur durch einen Arzt erfolgen. ${ }^{153}$ Im Entscheid nimmt das Bundesgericht explizit Bezug auf die medizin-ethischen Richtlinien der SAMW über die «Betreuung von Patienten am Lebensende». Gemäss diesen Richtlinien ${ }^{154}$ ist die Beihilfe zum Suizid nicht Teil der ärztlichen Tätigkeit, weil sie den Zielen der Medizin widerspreche; die Entscheidung einer einzelnen Ärztin, aufgrund einer persönlichen Gewissensentscheidung dennoch Beihilfe zum Suizid zu leisten, sei aber zu respektieren. In einem solchen Fall verlangen die Richtlinien die Prüfung der folgenden Voraussetzungen:

- Die Erkrankung des Patienten rechtfertigt die Annahme, dass das Lebensende nahe ist.

- Alternative Möglichkeiten der Hilfestellung wurden erörtert und soweit gewünscht auch eingesetzt.

- Die Patientin ist urteilsfähig, ihr Wunsch ist wohlerwogen, ohne äusseren Druck entstanden und dauerhaft. Dies wurde von einer unabhängigen Drittperson überprüft, wobei diese nicht zwingend ein Arzt sein muss.

Anders als die Richtlinien der SAMW sieht allerdings das Bundesgericht die Möglichkeit der ärztlichen Suizidbeihilfe nicht auf Patientinnen und Patienten am Lebensende beschränkt. Es schliesst sogar die ärztliche Beihilfe zum Suizid bei psychisch Kranken nicht generell aus, verlangt hier aber äusserste Zurückhaltung. 
Neben der Problematik der Suizidbeihilfe bei psychisch Kranken wirft in der Praxis auch das Verlangen auf Unterstützung von Personen im frühen Stadium einer Demenz sowie von betagten, lebensmüden, aber körperlich und psychisch weitgehend gesunden Personen schwierige Fragen auf, ohne dass darauf aber in der bisherigen Rechtsprechung eine klare Antwort zu finden wäre. Ein Positionspapier der SAMW vom März 2012 hält fest, dass die Richtlinien Suizidhilfe bei Menschen, die aufgrund allgemeiner Lebensmüdigkeit oder infolge fehlender Lebensqualität einen Sterbewunsch äussern, nicht erfassen, sofern diese nicht am Lebensende stehen. Eine zunehmende Etablierung der Suizidhilfe liege in der Verantwortung der Gesellschaft als Ganzer und dürfe nicht an die Ärzteschaft delegiert werden. Die grundsätzliche Diskussion über diese umstrittenen Fragen sei unerlässlich. ${ }^{155}$

Fälle von Suizidbeihilfe sind, gleich wie nichtassistierte Suizide, nichtnatürliche, also aussergewöhnliche Todesfälle mit entsprechender Meldepflicht (vgl. Kapitel 7.3.).

\subsection{Zwangsmassnahmen in der Medizin}

\section{Als Zwangsmassnahmen werden alle Eingriffe bezeichnet, die gegen den erklärten Willen oder gegen den Widerstand eines Menschen erfolgen. Darunter fallen auch weniger eingreifende Massnahmen, wie z.B. jemanden zu zwingen aufzustehen, Nahrung einzunehmen oder an einer therapeu- tischen Sitzung teilzunehmen.}

In der Praxis wird zwischen Freiheitsbeschränkung und Zwangsbehandlung unterschieden. Von Freiheitsbeschränkung spricht man, wenn ausschliesslich die Bewegungsfreiheit eingegrenzt wird (z.B. die Unterbringung in einer geschlossenen Abteilung). Schwerwiegende Freiheitsbeschränkungen sind die Fixation (z.B. mit Gurten) oder die Isolation (z.B. in einem Isolierzimmer). Wird nicht nur die Freiheit beschränkt, sondern auch in die körperliche Integrität eines Menschen eingegriffen, etwa bei einer Zwangsmedikation, handelt es sich um eine medizinische Zwangsmassnahme mit Verletzung der körperlichen Integrität. Dafür wird der Begriff Zwangsbehandlung verwendet.

Zwangsmassnahmen stellen in jedem Fall einen schweren Eingriff in das Selbstbestimmungsrecht und das Recht auf persönliche Freiheit eines Menschen dar. Dennoch sind Zwangsmassnahmen als letztes Mittel nicht immer zu umgehen. Bei akuter Selbst- oder Fremdgefährdung stellen sie manchmal die einzige zur Verfügung stehende Möglichkeit dar, um grösseren Schaden abzuwenden. 
In Notfallsituationen mit einem hohen Grad an Selbst- und Fremdgefährdung ist die Notwendigkeit von Zwangsmassnahmen kaum bestritten. Schwieriger ist die Ausgangslage in Situationen ohne Notfallcharakter, bei denen Aspekte der Sicherheit oder Gesundheitsschädigung im Vordergrund stehen, insbesondere im Bereich der Altersmedizin und Psychiatrie. Hier ist oft nicht eindeutig, ob das Prinzip «Gutes tun» die Einschränkung der Persönlichkeitsrechte und Freiheit, also die punktuelle Durchbrechung der Patientenautonomie, tatsächlich aufwiegt.

Zwangsmassnahmen können ausserordentlich traumatisierend sein. Daher ist das Prinzip der Verhältnismässigkeit besonders zu achten; das heisst, eine solche Massnahme muss erstens notwendig, zweitens proportional zur Schwere der Gefährdung und drittens nicht durch weniger einschneidende Massnahmen ersetzbar sein. Es ist deshalb im Einzelfall zu prüfen, welche Massnahme für den Betroffenen am wenigsten belastend ist. Zudem ist abzuschätzen, ob der zu erwartende Nutzen den möglichen Schaden eines solchen Eingriffes deutlich übertrifft bzw. weniger gravierende Folgen hat als eine sonst notwendige Massnahme. Auch die Dauer der Zwangsmassnahme ist den Umständen anzupassen. Zudem muss eine Zwangsmassnahme nach bestem Stand des Wissens ausgewählt und reversibel sein.

\section{Fürsorgerische Unterbringung (FU)}

Eine Zwangseinweisung darf nur erfolgen, wenn die Voraussetzungen einer fürsorgerischen Unterbringung (FU) erfüllt sind oder eine andere klare gesetzliche Grundlage (z.B. Epidemiengesetz) besteht. Eine schutzbedürftige Person darf in einer geeigneten Einrichtung untergebracht werden, wenn sie «an einer psychischen Störung oder an einer geistigen Behinderung leidet oder schwer verwahrlost ist» und «die nötige Behandlung oder Betreuung nicht anders erfolgen kann». ${ }^{156}$ Die FU ist ein schwerer Eingriff in das Grundrecht der persönlichen Freiheit, weshalb sie nur als Ultima Ratio in Frage kommt. Für die Unterbringung und die Entlassung ist die Erwachsenenschutzbehörde zuständig. Die Kantone können die Einweisungskompetenz auch an Ärzte und Ärztinnen delegieren, wobei die Dauer der Unterbringung in diesem Fall auf maximal sechs Wochen zu beschränken ist. ${ }^{157}$ Die untergebrachte Person hat das Recht, eine Vertrauensperson beizuziehen (mehr dazu in Kap. 3.).

Erfolgt die Unterbringung zum Zwecke der Behandlung, so erstellt die behandelnde Ärztin oder der behandelnde Arzt unter Beizug der betroffenen Person und der Vertrauensperson einen Behandlungsplan. Ist die betroffene Person urteilsfähig, so muss ihr der Behandlungsplan zur Zustimmung unterbreitet werden. 
Es gelten in diesem Fall die Grundsätze der informierten Einwilligung (informed consent). Ist die untergebrachte Person urteilsunfähig, so ist eine allfällige Patientenverfügung (mehr dazu Kap. 4.4.) zu berücksichtigen. Auch bei FU muss die Patientenverfügung somit nach Möglichkeit befolgt werden, auch wenn ihr keine absolute Verbindlichkeit zukommt. Die Zwangsbehandlung ist im Gesetz unter dem Titel «Behandlung ohne Zustimmung» geregelt. ${ }^{158}$ Sie ist durch den Chefarzt oder die Chefärztin schriftlich anzuordnen und setzt insbesondere voraus, dass die betroffene Person hinsichtlich ihrer Behandlungsbedürftigkeit urteilsunfähig ist und eine ernsthafte Gefahr für ihre Gesundheit oder das Leben oder die körperliche Integrität Dritter besteht. Liegt eine Notfallsituation vor, so können zudem die zur Abwendung der akuten Gefahrensituation unerlässlichen medizinischen Massnahmen sofort ergriffen werden. Diese Regeln sind nur auf die Behandlung von psychischen Störungen bei FU anwendbar. Bei somatischen Erkrankungen sind auch während einer FU die medizinischen Vertretungsrechte bei Urteilsunfähigen zu beachten (mehr dazu siehe Kap. 3. und 4.3.). Auf freiheitsbeschränkende Massnahmen (Fixation, Isolation) sind die Bestimmungen über die Einschränkung der Bewegungsfreiheit in Wohn- und Pflegeeinrichtungen bei FU sinngemäss anwendbar (mehr dazu siehe Kap. 5.7.).

Bei FU besteht ein ausgebauter Rechtsschutz. Die betroffene oder eine ihr nahestehende Person kann das Gericht in folgenden Fällen anrufen: Anordnung der ärztlichen Unterbringung, Zurückbehaltung und Abweisung eines Entlassungsgesuches durch die Einrichtung, Behandlung ohne Zustimmung, Einschränkung der Bewegungsfreiheit. Die Frist zur Anrufung des Gerichts beträgt grundsätzlich zehn Tage seit Mitteilung des Entscheides.

Das neue Erwachsenenschutzrecht hat darauf verzichtet, Rechtsgrundlagen für die ambulante Zwangsbehandlung einzuführen. Aufgrund eines ausdrücklichen Vorbehaltes haben jedoch die Kantone die Befugnis, in diesem Bereich zu legiferieren. Sie können die Nachbetreuung regeln und ambulante Massnahmen vorsehen. Zahlreiche Kantone haben von dieser Kompetenz Gebrauch gemacht. Danach wird in der Regel der Erwachsenenschutzbehörde die Befugnis erteilt, der betroffenen Person die Auflage zu machen, sich einer ärztlichen Behandlung zu unterziehen oder medizinisch indizierte Medikamente einzunehmen. Auf diese Weise sollen FU verhindert bzw. Rückfälle vermieden werden. 


\section{Indirekter Behandlungszwang durch Strafrichter oder Versicherer}

Gemäss Strafgesetzbuch kann ein Strafrichter bei psychischen Störungen oder einer Suchtmittelabhängigkeit des Täters eine stationäre therapeutische Massnahme oder eine ambulante medizinische Behandlung anordnen. ${ }^{159}$ Bei ambulanten Therapien besteht immerhin ein erheblicher Druck zur Mitwirkung, da bei einem Therapieabbruch der Vollzug einer aufgeschobenen Freiheitsstrafe droht.

In verschiedenen Versicherungsbereichen sind Versicherte zudem verpflichtet, den Schaden zu mindern. Es besteht damit ein indirekter Zwang, sich behandeln zu lassen: Unfallversicherer, Militärversicherung und die IV können verlangen, dass sich jemand zumutbaren Behandlungen - auch invasiven Eingriffen - unterzieht, wenn damit seine Erwerbsfähigkeit verbessert werden kann. Und sogar die private Taggeldversicherung kann verlangen, dass sich ein Versicherter zumutbar medizinisch behandeln lässt, wenn dies der Wiederherstellung seiner Arbeitsfähigkeit dient. Selbstverständlich ersetzt dieser indirekte Zwang durch den Versicherer nicht die Zustimmung des Patienten zur Behandlung; der Patient kann «Nein» sagen und in Kauf nehmen, dass die Versicherung ihre Taggeld- oder Rentenleistungen streicht oder kürzt.

\subsection{Behandlung von Patienten im Strafvollzug}

\section{Die Grundrechte des Bürgers gelten auch für die Mitwirkung von Ärzten im Strafvollzug, sei dies bei polizeilichen Zwangsmassnahmen (z. B. Ausschaffungen) oder in der medizinischen Betreuung von Inhaftierten.}

Verschiedene internationale Organisationen (UNO, Europarat usw.) haben in den letzten Jahren Leitlinien für die gesundheitliche Betreuung von inhaftierten Personen verfasst. Auch die SAMW hat 2002 Richtlinien für die «Ausübung der ärztlichen Tätigkeit bei inhaftierten Personen» verabschiedet, die von der FMH in die Standesordnung aufgenommen wurden. ${ }^{160}$

Menschen im Strafvollzug haben grundsätzlich dieselben Rechte bezüglich ihrer Gesundheit wie alle anderen Bürger. Sie haben Anrecht auf eine Behandlung, die medizinisch jener der Allgemeinbevölkerung entspricht. ${ }^{161}$ Wie in jedem Arzt-

159 Art. 56ff StGB.

160 Die SAMW hat im Januar 2012 zusätzlich «Hinweise zur praktischen Umsetzung der Richtlinien» veröffentlicht.

161 Vgl. auch Art. 80, Abs. 1 StGB: «Von den für den Vollzug geltenden Regeln darf zu Gunsten des Gefangenen abgewichen werden: a. wenn der Gesundheitszustand des Gefangenen dies erfordert; b. bei Schwangerschaft, Geburt und für die Zeit unmittelbar nach der Geburt; c. zur gemeinsamen Unterbringung von Mutter und Kleinkind, sofern dies auch im Interesse des Kindes liegt." 
Patienten-Verhältnis darf auch im Strafvollzug eine diagnostische oder therapeutische Massnahme nur nach Aufklärung und mit dem freien Einverständnis der inhaftierten Person durchgeführt werden. Zudem besteht auch hier ein Recht auf Vertraulichkeit und Datenschutz.

In Notfallsituationen kann die Ärztin - nach den gleichen Kriterien, die für nichtinhaftierte Personen gelten - auf die Einwilligung verzichten. Dies gilt z.B. für medizinisch begründete Massnahmen zur physischen Ruhigstellung, die jedoch höchstens für einige wenige Stunden und unter regelmässiger Beobachtung in Betracht gezogen werden dürfen. Ist der Patient aber urteilsfähig, muss sein Wille respektiert werden. Dies gilt auch für einen Hungerstreik, der zu einem beträchtlichen Gesundheitsrisiko für den Betroffenen führen kann. Fällt die hungerstreikende Person in ein Koma, geht die Ärztin nach ihrem Gewissen und ihrer Berufsethik vor, es sei denn, die betreffende Person habe ausdrückliche Anordnungen für den Fall eines Bewusstseinsverlusts hinterlegt. Solche sind zu respektieren, auch wenn sie den Tod zur Folge haben können. Die Frage ist allerdings, ob der Todeswunsch ernsthaft ist, will doch der Hungerstreikende in der Regel ein bestimmtes Ziel erreichen und nicht sein Leben beenden. Auch unter Juristen stiess der Entscheid des Bundesgerichts betreffend Bernard Rappaz auf Kritik: Es wollte die Ärzte verpflichten, am Hungerstreikenden eine Zwangsernährung nach den Regeln der Kunst und unter Beachtung der Menschenwürde anzuwenden ${ }^{162}$ nicht nur aus ärztlicher Sicht ein unauflösbarer Widerspruch.

Die ärztliche Schweigepflicht gemäss Art. 321 StGB besteht grundsätzlich auch gegenüber dem Überwachungspersonal einer Haftanstalt. Aus diesem Grund müssen die Krankengeschichten unter ärztlicher Verantwortung aufbewahrt werden. Ausnahmen von der Schweigepflicht sind zum Schutz vor der Übertragung ansteckender Krankheiten oder bei unmittelbarer Gefahr von selbst- oder fremdgefährdenden Handlungen der inhaftierten Person zulässig. Hier ist aber jeder Fall einzeln zu beurteilen.

Konflikte können entstehen, wenn ein Arzt Zeuge von schwerwiegenden disziplinarischen Massnahmen (z. B. Isolation) wird oder gar Misshandlungen feststellt. Disziplinarische Massnahmen dürfen nur durchgeführt werden, wenn sie mit dem Erhalt der Gesundheit vereinbar sind; Hinweise auf Misshandlungen müssen vom behandelnden Arzt festgehalten und den Aufsichtsbehörden gemeldet werden. Ein Spannungsfeld kann auch zwischen Strafauftrag des Gefängnisses und Gesundheitsschutz bestehen, wenn Desinfektionsmaterial und Einwegspritzen zur Risikominderung bei Suchtmittelkonsum zur Verfügung gestellt werden. Aus grundsätzlichen Überlegungen muss sich deshalb die Ärztin gegenüber polizeilichen oder Strafvollzugsbehörden auf volle Unabhängigkeit berufen können und ihre Entscheidungen ausschliesslich auf medizinische Kriterien stützen. 


\subsection{Behandlung von Sans-Papiers und Asylsuchenden}

Auch Sans-Papiers und Asylsuchende haben einen Anspruch auf Wahrung des

Arztgeheimnisses. Asylsuchende sind obligatorisch krankenversichert, müssen aber in einem Managed-Care-Modell betreut werden. Abgewiesene Asylsuchende und solche mit Nichteintretensentscheid sind nicht KVGversichert, sondern erhalten nur eine Mindestversorgung.

Asylsuchende im laufenden Verfahren sind KVG-versichert, müssen aber vom Kanton einem Managed-Care-Modell zugewiesen werden. ${ }^{163}$ Asylsuchende, auf deren Gesuch nicht eingetreten wurde (sogenannte NEE), und abgewiesene Asylbewerber sind nach der letzten Asylgesetzrevision nicht mehr krankenversichert. Für sie gilt lediglich, dass die Kantone im Rahmen des Existenzminimums die medizinische Mindestversorgung sicherstellen müssen, wofür sie eine Pauschale des Bundes erhalten.

Für die medizinische Betreuung im Zusammenhang mit der Rückführung von abgewiesenen Asylsuchenden gelten aus berufsethischer und standesrechtlicher Sicht die Richtlinien der SAMW von 2002 über die «Ausübung der ärztlichen Tätigkeit bei inhaftierten Personen». Der FMH-Zentralvorstand hat im Juni 2012 daran erinnert, dass die mit der medizinischen Betreuung beauftragten Ärztinnen und Ärzte in der Lage sein müssen, ihre Rolle korrekt wahrzunehmen. Dazu gehört insbesondere die Kenntnis der Krankengeschichte, die Möglichkeit, die auszuschaffende Person vorher zu untersuchen, und die Möglichkeit einer wirksamen Überwachung während des Transports (unter anderem muss der Arzt das Gesicht erkennen und den Puls messen können). Zudem müssen die mit der medizinischen Betreuung beauftragten Ärztinnen und Ärzte erfahren und unabhängig sein. Der Abschluss von Verträgen zur medizinischen Begleitung mit grossen Gruppenpraxen, die dann ihre jüngeren ärztlichen Mitarbeiterinnen schicken, kann dies nicht sicherstellen.

163 Art. 82 revidiertes Asylgesetz. Siehe auch «Krankenversicherung und Zugang zur Gesundheitsversorgung von Sans Papiers" Bericht des Bundesrates in Erfüllung des Postulates Heim (09.3484) vom Mai 2012. (www.news.admin.ch/message/index.html?lang=de\&msg-id=44651). 


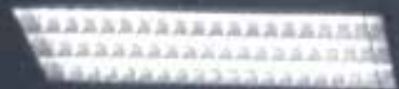

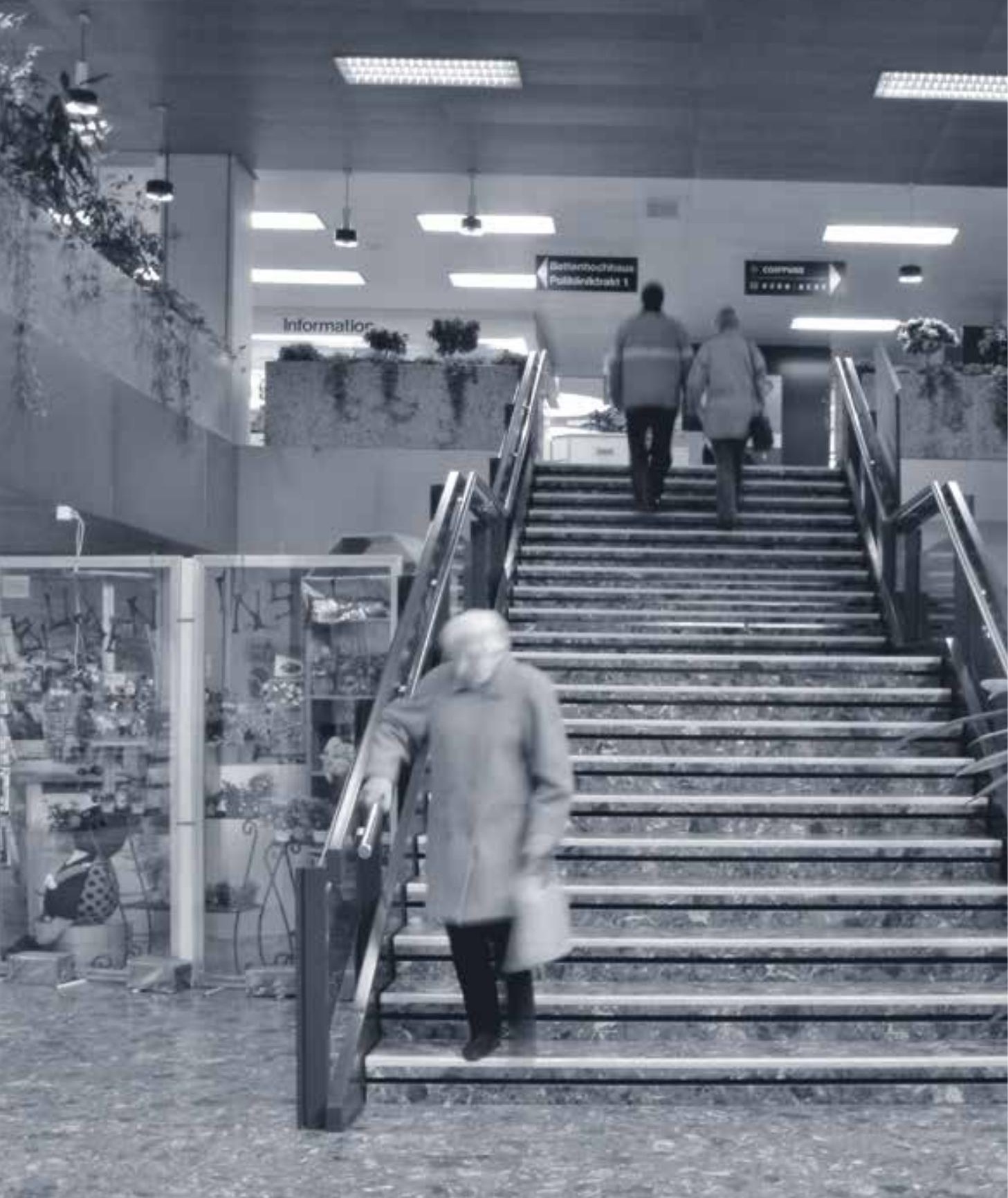




\section{Forschung}

\subsection{Forschung am Menschen}

Mit dem im März 2010 von Volk und Ständen angenommenen Verfassungsartikel Art. 118b BV wurde der Bund ermächtigt, die Forschung am Menschen umfassend zu regeln. Gestützt darauf hat das Parlament das Humanforschungsgesetz verabschiedet, das zusammen mit dem Verordnungsrecht voraussichtlich 2014 in Kraft treten wird. Bis zu diesem Zeitpunkt gilt für Heilmittelstudien nach wie vor das Heilmittelgesetz sowie für Forschungsvorhaben in spezifischen Teilbereichen die einschlägigen Bestimmungen im Bundesrecht und in kantonalen Gesetzen.

Das Humanforschungsgesetz regelt die Forschung zu Krankheiten des Menschen sowie zu Aufbau und Funktion des menschlichen Körpers umfassend. Vom Geltungsbereich erfasst sind alle Forschungsprojekte mit lebenden und verstorbenen Personen, an Embryonen und Föten sowie mit biologischem Material menschlicher Herkunft (Biomaterial) und mit gesundheitsbezogenen Personendaten, sofern das Biomaterial bzw. die Daten nicht anonymisiert sind.

Zu den zentralen Grundregeln gehören gemäss Humanforschungsgesetz die folgenden:

- Interesse, Gesundheit und Wohlergehen des einzelnen Menschen haben Vorrang gegenüber den Interessen der Wissenschaft und der Gesellschaft (Art. 4).

- Grundsätzlich darf eine Person nur dann in ein Forschungsprojekt einbezogen werden, wenn sie nach hinreichender Aufklärung in die Teilnahme eingewilligt hat. Die Zustimmung muss freiwillig erfolgen und die betroffene Person kann ihre Einwilligung jederzeit ohne Begründung widerrufen (Art. 7).

- Für Forschungsprojekte mit Personen, die noch nicht volljährig sind oder die aufgrund mangelnder Urteilsfähigkeit oder einer spezifischen Situation (z.B. Notfallsituation) einer besonderen Aufmerksamkeit bedürfen (sogenannte besonders verletzbare Personen), gelten zusätzliche Einwilligungsvoraussetzungen und Schutzvorschriften (Art. $21 \mathrm{ff}$.).

- Jegliche Forschung muss den relevanten wissenschaftlichen Anforderungen genügen, ebenso sind die Prinzipien der wissenschaftlichen Integrität zu respektieren (Art. 10). 
- Forschungsprojekte mit Personen, insbesondere klinische Versuche, dürfen nur durchgeführt werden, wenn gleichwertige Erkenntnisse anders nicht gewonnen werden können (Art. 11). Bei jedem Forschungsprojekt sind die voraussichtlichen Risiken und Belastungen für die teilnehmenden Personen so gering wie möglich zu halten; diese dürfen in keinem Missverhältnis zum erwarteten Nutzen des Forschungsprojekts stehen (Art. 12).

- Wer ein Forschungsprojekt durchführt, muss alle erforderlichen Massnahmen zum Schutz der teilnehmenden Person treffen (Art. 15).

- Personen, die an einem Forschungsprojekt teilnehmen, haben das Recht, über die ihre Gesundheit betreffenden Ergebnisse informiert zu werden. Sie können auf diese Information aber auch verzichten (Art. 8).

- Die unabhängige Überprüfung der Forschungsprojekte erfolgt wie bis anhin durch eine kantonale Ethikkommission für die Forschung (Art. 45).

- Schliesslich unterliegen klinische Versuche einer Registrierungspflicht in einem anerkannten Register (Art. 56).

Nicht vom Humanforschungsgesetz erfasst ist die Durchführung einer nichtetablierten Behandlung bei einem einzelnen Patienten (sogenannter «Heilversuch», vgl. Kapitel 4.8.). Ebenso ist Qualitätssicherung ohne das Ziel Erkenntnisgewinn keine Forschung und deshalb nicht von der Ethikkommission zu genehmigen (vgl. Kap. 4.13.).

\subsection{Stammzellenforschung}

Forschung mit embryonalen Stammzellen ist zulässig, wenn sie wesentliche Erkenntnisse über schwere Krankheiten oder die Entwicklungsbiologie des Menschen erhoffen lässt.

Die Forschung mit embryonalen Stammzellen ist sowohl mit importierten als auch mit hierzulande gewonnenen Stammzellen möglich, bedarf aber einer positiven Stellungnahme der zuständigen Ethikkommission. Diese überprüft vor allem, ob mit dem Projekt wesentliche Erkenntnisse über schwere Krankheiten oder die Entwicklungsbiologie des Menschen erzielt werden können und ob die wissenschaftlichen Qualitätsanforderungen erfüllt sind. Auch dürfen nur Stammzellen verwendet werden, die in der Schweiz oder im Ausland mit dem «informed consent» des betreffenden Paares aus sogenannten «überzähligen», ursprünglich zu Fortpflanzungszwecken erzeugten Embryonen, gewonnen wurden. ${ }^{164}$

Der Import von Stammzellen sowie die Gewinnung von embryonalen Stammzellen aus überzähligen Embryonen sind bewilligungspflichtig (BAG). Das Gesetz

164 Überzählig ist ein Embryo dann, wenn er im Rahmen eines In-vitro-Fertilisationsverfahrens erzeugt wurde, er aber (z.B. aufgrund der Entscheidung der Frau) nicht mehr zur Herbeiführung einer Schwangerschaft verwendet wird. Verboten ist demgegenüber die Erzeugung eines Embryos eigens zu Forschungszwecken oder die Bildung eines Klons. 
verlangt dabei eine klare Trennung der fortpflanzungsmedizinischen Behandlung und der Gewinnungs- bzw. Forschungstätigkeiten; insbesondere dürfen Personen im konkreten Fall nicht an beiden Aktivitäten beteiligt sein. Die Stammzellengewinnung ist zudem nur zulässig, wenn ein konkretes Forschungsprojekt geplant ist, hierfür aber in der Schweiz keine geeigneten Zellen vorliegen. ${ }^{165}$

\subsection{Wissenschaftliche Integrität}

\section{"Wissenschaftliche Integrität» umschreibt die Selbstverpflichtung der Forschenden, sich an die Grundregeln der guten wissenschaftlichen Praxis zu halten. Um sie sicherzustellen, wurden verschiedene nationale und internationale Reglemente ausgearbeitet.}

Fairness, Wahrhaftigkeit und Offenheit sowie Selbstdisziplin sind Grundlagen für jede wissenschaftliche Tätigkeit. Wissenschaftliche Integrität ist unerlässlich für die Glaubwürdigkeit und Akzeptanz der Wissenschaft.

Verstösse gegen die Grundregeln der guten wissenschaftlichen Praxis sind in vielfältiger Weise möglich: Von der mangelnden Sorgfalt bei der Anwendung wissenschaftlicher Methoden oder bei der Dokumentation von Daten bis hin zu schwerem Fehlverhalten durch bewusste Fälschung und Betrug, vom Plagiat über Datenpiraterie bis hin zur Sabotage. Wissenschaftliches Fehlverhalten kann sowohl in der Konzeption oder Durchführung eines Projektes als auch bei wissenschaftlichen Gutachten oder bei der Beurteilung von Forschungsgesuchen und Forschungsergebnissen vorkommen.

165 Die Website des BAG (www.bag.admin.ch/themen/medizin/03301/index.html?lang=de) enthält sowohl die gesetzlichen Grundlagen als auch einen Überblick über die Voraussetzungen und Bewilligungsprozesse und das Forschungsregister. 
Um die wissenschaftliche Integrität sicherzustellen, haben diverse Forschungsinstitutionen und Institutionen der Forschungsförderung im In- und Ausland Reglemente ausgearbeitet. ${ }^{166}$ Diese halten die wesentlichen Grundsätze der wissenschaftlichen Integrität fest und regeln den Umgang mit wissenschaftlichem Fehlverhalten. Wird der Verdacht auf wissenschaftliches Fehlverhalten von einem sogenannten «Whistleblower» geäussert, muss dieser vor allfälligen Repressalien oder Benachteiligungen geschützt werden.

Die meisten Institutionen verfügen über eine Ombudsperson, die eine Beratungs- und Schlichtungsfunktion übernimmt und Anzeigen betreffend wissenschaftlichem Fehlverhalten entgegennimmt. Ist ein Fehlverhalten nicht auszuschliessen, prüft eine «Integritätsschutz-Organisation», ob ein Verstoss gegen die wissenschaftliche Integrität vorliegt und wie dieser sanktioniert werden soll. Falls das Fehlverhalten gegen einschlägige staatliche Rechtsvorschriften verstösst und es darum geht, entsprechende Rechtsansprüche gegen den Fehlbaren durchzusetzen, wird gleichzeitig mit der Anzeige beim Ombudsmann eine Anzeige oder Klage bei den zuständigen staatlichen Gerichten notwendig.

Die Zusammenarbeit von klinischen Forscherinnen und Forschern mit der Industrie oder mit von ihr beauftragten Forschungsinstituten stellt zusätzliche Anforderungen an die wissenschaftliche Integrität. Die Aussicht, mit Forschungsergebnissen Vorteile zu erlangen, kann Forschende dazu verleiten, bei der Planung, Durchführung oder Auswertung von Studien inkorrekt zu handeln. Die SAMW hat deshalb Richtlinien erlassen, die zur Qualität und Objektivität der Forschung, zur Vermeidung von Abhängigkeiten und zum bewussten Umgang mit Interessenkonflikten beitragen sollen. ${ }^{167}$

166 Die Schweizerische Akademie der Medizinischen Wissenschaften hat 2002 Richtlinien für wissenschaftliche Integrität in der medizinischen und biomedizinischen Forschung und für das Verfahren bei Fällen von Unlauterkeit verabschiedet. Diese wurden 2008 durch Richtlinien der Akademien der Wissenschaften Schweiz abgelöst. Gleichzeitig haben die Akademien der Wissenschaften Schweiz "Grundsätze und Verfahrensregeln» zur wissenschaftlichen Integrität veröffentlicht, die sich an Forschende, Forschungsinstitutionen sowie Institutionen der Forschungsförderung richten. Vergleichbare Bestimmungen bestehen für andere Wissenschaftsbereiche. Mittlerweile verfügt die Mehrheit der schweizerischen Universitäten und Hochschulen sowie der Schweizerische Nationalfonds über eigene Reglemente zur Integrität und zum Umgang mit Fehlverhalten.

167 «Zusammenarbeit Ärzteschaft - Industrie», Richtlinien der SAMW. 


\section{Der Arzt als Berichterstatter: vom Arztgeheimnis zur Rechnungs- stellung}

\subsection{Arztgeheimnis (Berufsgeheimnis, Patientengeheimnis)}

Angehörige von Gesundheitsberufen sind wie Anwälte oder Geistliche an das Berufsgeheimnis gebunden. Unter die Geheimhaltungspflicht fällt alles, was den Ärztinnen und Ärzten in der Berufsausübung anvertraut worden ist oder was sie in dessen Ausübung wahrgenommen haben. Ein Arzt darf Dritten Auskunft geben, wenn die Einwilligung der Patientin dazu vorliegt, ein Gesetz dies vorsieht oder er von der kantonalen Behörde vom Geheimnis entbunden wurde.

Der traditionelle Begriff «Arztgeheimnis» hat seine Berechtigung. Er macht bewusst, dass die Ausübung des Arztberufs nur möglich ist, wenn Arzt und Ärztin die Vertraulichkeit wahren können. Wer das Arztgeheimnis aufs Spiel setzt, nimmt dem Patienten die Möglichkeit, sich ohne Bedenken anvertrauen zu können, und zerstört damit den Arztberuf. Der Begriff «Patientengeheimnis» betont, dass es um den Schutz der Persönlichkeitsrechte der Patientin geht. Auch diese Perspektive ist zutreffend. Es kommt auf den Kontext an. Geregelt ist das «ärztliche Berufsgeheimnis» - so der gesetzliche Begriff - in Art. 321 des Strafgesetzbuchs (vgl. Kasten).

Unter die Geheimhaltungspflicht fällt alles, was Ärztin und Arzt «infolge ihres Berufes anvertraut worden ist, oder das sie in dessen Ausübung wahrgenommen haben»: Geheim ist somit, was Patienten oder deren Angehörige der Ärztin anvertrauen, aber auch alles, was der Arzt selbst im Rahmen seiner Tätigkeit wahrnimmt und tut, etwa die Untersuchungsergebnisse, die resultierende Diagnose und die Behandlungen.

Nicht wenige Patienten zahlen die Arztrechnung selbst, sei dies im Zusammenhang mit höheren Jahresfranchisen oder aus spezifischen, z.B. beruflichen oder familiären Geheimhaltungsgründen. Auch für eine online Abfrage der Arztpraxis bei der Krankenkasse braucht es deshalb das Patienteneinverständnis, weil damit die Kasse von Anfang an weiss, dass der Patient bei diesem Arzt in Behandlung ist.

Der Patient hat auch das Recht, frei zu entscheiden, ob er die Versichertenkarte vorweisen will, oder ob er in Kauf nimmt, dass seine Kasse allenfalls eine angemessene Bearbeitungsgebühr verlangt, wenn AHV- und Versichertenkartennum- 
mer nicht auf der Rechnung stehen. ${ }^{168}$ Die gesetzliche Leistungspflicht der Kasse hängt nicht davon ab, ob die AHV- und Versichertenkartennummer auf der Rechnung stehen.

Auch wenn der Arzt Laboraufträge extern vergibt und das Labor im «tiers payant» (t.p.) abrechnet, sollte er den Patienten vorgängig fragen, ob dieser die Abrechnung mit t.p. akzeptiert oder die Rechnung selbst erhalten will. Nach Auffassung der FMH gehört auch die ärztliche Gutachtertätigkeit zur Ausübung des Arztberufs und untersteht somit dem Patientengeheimnis (siehe Kap. 8.1.): Selbst wenn der Arzt bloss ein Aktengutachten erstellt und die Patientin gar nicht sieht, darf er dieses beispielsweise nicht den Medien weitergeben.

Nicht unter das Patientengeheimnis fallen hingegen bereits allgemein bekannte oder offenkundige Tatsachen - auch wenn die Ärztin diese in ihrer Berufsausübung wahrgenommen hat.

Neben den Ärztinnen und Ärzten untersteht auch das Personal der Arztpraxis und der Spitäler dem Patientengeheimnis. Der Arzt soll seine Hilfspersonen ausdrücklich darauf aufmerksam machen. ${ }^{169}$

168 Art. 10 Abs. 2 VVK: "Weist die versicherte Person die Versichertenkarte nicht vor und verursacht sie dadurch zusätzliche Aufwendungen bei der Vergütung von Leistungen, so kann der Versicherer eine angemessene Gebühr erheben."

169 Art. 11 Abs. 2 FMH-Standesordnung: "Arzt und Ärztin haben ihre Mitarbeiter und Mitarbeiterinnen und alle, die in ihre Praxis Einblick erhalten, über die Pflicht zur Verschwiegenheit zu informieren und sie nach Möglichkeit schriftlich auf deren Einhaltung zu verpflichten.» 


\section{Das Berufsgeheimnis im Wortlaut}

Artikel 321 Strafgesetzbuch

1. Geistliche, Rechtsanwälte, Verteidiger, Notare, nach Obligationenrecht zur Verschwiegenheit verpflichtete Revisoren, Ärzte, Zahnärzte, Apotheker, Hebammen sowie ihre Hilfspersonen, die ein Geheimnis offenbaren, das ihnen infolge ihres Berufes anvertraut worden ist, oder das sie in dessen Ausübung wahrgenommen haben, werden, auf Antrag, mit Freiheitsstrafe bis zu drei Jahren oder Geldstrafe bestraft.

Ebenso werden Studierende bestraft, die ein Geheimnis offenbaren, das sie bei ihrem Studium wahrnehmen.

Die Verletzung des Berufsgeheimnisses ist auch nach Beendigung der Berufsausübung oder der Studien strafbar.

2. Der Täter ist nicht strafbar, wenn er das Geheimnis auf Grund einer Einwilligung des Berechtigten oder einer auf Gesuch des Täters erteilten schriftlichen Bewilligung der vorgesetzten Behörde oder Aufsichtsbehörde offenbart hat.

3. Vorbehalten bleiben die eidgenössischen und kantonalen Bestimmungen über die Zeugnispflicht und über die Auskunftspflicht gegenüber einer Behörde.

Es gibt vier Gründe, die es rechtfertigen, eine dem Berufsgeheimnis unterstehende Information weiterzugeben:

1. die Einwilligung des Patienten;

2. eine Ausnahmebestimmung in einem Bundesgesetz oder kantonalen Gesetz;

3. die Entbindung durch die kantonale Gesundheitsdirektion;

4. eine akute Notstandssituation gemäss Art. 17f und 48a Strafgesetzbuch.

Diese Rechtfertigungsgründe werden im Folgenden kurz erläutert.

\section{Auskunftserteilung aufgrund der Einwilligung des Patienten}

Die Auskunftserteilung aufgrund der Einwilligung des Patienten ist der Normalfall. Für sie gelten die Regeln des «informed consent», das heisst, damit die Einwilligung gilt, muss der Patient genügend wissen, welche Informationen die Ärztin dem Dritten mitteilen wird. Der Patient kann sein Einverständnis schriftlich oder mündlich erteilen; auch die stillschweigende und sogar die mutmassliche Einwilligung genügen. Wegen der möglichen Missverständnisse sollte die Ärztin sich nicht ohne Not auf die stillschweigende oder mutmassliche Einwilligung 
stützen (vgl. Kap. 4.3.). Wenn der Patient seine Ärztin im Hinblick auf eine Zeugenaussage vor Gericht vom Berufsgeheimnis entbindet, muss der Arzt gemäss ZPO und StPO aussagen, es sei denn, er könne «glaubhaft machen, dass das Geheimhaltungsinteresse das Interesse an der Wahrheitsfindung überwiegt». ${ }^{170}$

Das Patientengeheimnis gilt auch gegenüber Angehörigen: Es ist sinnvoll, routinemässig den Patienten zu fragen, welche seiner Angehörigen man gegebenenfalls über seinen Gesundheitszustand informieren darf oder soll. Ein gesetzliches Informationsrecht über gesundheitliche Fragen haben hingegen die Eltern urteilsunfähiger Kinder (auch Elternteile, die das Sorgerecht für ein Kind nicht haben). Ist das Kind bzw. der Jugendliche für die Behandlung urteilsfähig (vgl. Kap. 4.3.), ist allerdings sein Patientengeheimnis zu wahren.

Auskünfte am Telefon sind zwar erlaubt; der Arzt muss aber soweit möglich sicherstellen, dass er nur berechtigten Personen Auskunft gibt. Wenn man Anfragende nicht sicher an der Stimme erkennt, soll man sich die Telefonnummer geben lassen, wenn möglich die Nummer überprüfen und zurückrufen.

Für die Rechnungsstellung durch einen Dritten, etwa eine Ärztekasse oder andere Dienstleister, muss die Arztpraxis die einzelnen Leistungen übermitteln. Aufgrund der Tarifpositionen können sich fachkundige Dritte ein weitgehendes Bild über die Behandlung machen. Deshalb ist es wichtig, der Patientin von Anfang an mitzuteilen, bei wem man die Rechnung auswärts erstellen lässt. Wenn dies für die Patientin ein Problem ist, kann man mit ihr im Einzelfall eine andere Lösung suchen.

\section{Auskunftserteilung aufgrund gesetzlicher Entbindungsbestimmungen}

Auch wenn dies der Patient möglicherweise oder gar explizit nicht will, haben Ärztinnen und Ärzte in bestimmten Fällen das Recht oder sogar die Pflicht, Dritte zu informieren (vgl. Kap. 7.3.). So enthalten alle Sozialversicherungsgesetze Bestimmungen, die den Arzt berechtigen und verpflichten, der Sozialversicherung die Informationen zukommen zu lassen, die diese benötigt, um ihren gesetzlichen Auftrag zu erfüllen. Im Krankenversicherungsgesetz ist allerdings die Patienteneinwilligung entscheidend, denn der Patient kann entscheiden, die Behandlung selbst zu bezahlen; in diesem Fall darf die Krankenkasse vom Arzt nichts erfahren. 


\title{
Entbindung durch die kantonale Gesundheitsdirektion
}

Wenn eine Information weitergegeben werden sollte, aber der Patient nicht einwilligt, kann ein Arzt der zuständigen kantonalen Behörde den Antrag stellen, vom Berufsgeheimnis entbunden zu werden. Zuständig ist in der Regel die kantonale Gesundheitsdirektion. Einige Kantone haben spezielle Kommissionen dafür eingesetzt. Empfehlenswert ist, den Fall noch ohne Nennung von Patientennamen und anderen Informationen, die den Patienten identifizieren, kurz am Telefon mit der Kantonsärztin oder dem Rechtsdienst der Gesundheitsdirektion vorzubesprechen.

\section{Auskunftserteilung in einer Notstandssituation}

Gemäss Strafgesetzbuch darf das Berufsgeheimnis verletzt werden, wenn es darum geht, «ein eigenes oder das Rechtsgut einer anderen Person aus einer unmittelbaren, nicht anders abwendbaren Gefahr zu retten». ${ }^{171}$ Ein Notstand in diesem Sinn kann nur vorliegen, wenn die Zeit nicht mehr reicht, entweder die Einwillgung des Patienten einzuholen oder die Gesundheitsdirektion bzw. den Kantonsarzt zu kontaktieren, um eine Entbindung vom Berufsgeheimnis zu beantragen. In der Praxis ist dies also ein seltener Fall.

\subsection{Datenschutz}

\begin{abstract}
Das Datenschutzgesetz des Bundes schützt die Persönlichkeit und regelt die Rechte von Personen, deren Daten bearbeitet werden. Es gilt für alle Privaten - also auch für die Arztpraxen und Privatspitäler -, für die Bundesverwaltung, aber auch für die ausgelagerte Bundesverwaltung wie beispielsweise die Krankenkassen und die Unfallversicherer. Das künftige Bundesgesetz über das elektronische Patientendossier (EPDG) soll den elektronischen Informationsaustausch zwischen sogenannten Gemeinschaften von Leistungserbringern regeln.
\end{abstract}

\section{Datenschutz}

Für Ärztinnen und Ärzte schafft das Datenschutzgesetz (DSG) faktisch keine zusätzlichen Pflichten, da sie bereits dem Berufsgeheimnis unterstehen. Das DSG hält aber viele vernünftige Grundsätze fest:

- Die Datenbearbeitung muss verhältnismässig sein und nach dem Grundsatz von Treu und Glauben erfolgen. Personendaten dürfen nur zu dem Zweck bearbeitet werden, der bei der Beschaffung angegeben wurde, aus den Umständen ersichtlich oder gesetzlich vorgesehen ist. ${ }^{172}$ 
- Auch die Einwilligung der betroffenen Person rechtfertigt keine unverhältnismässige Datenbearbeitung, denn Personendaten dürfen nur rechtmässig bearbeitet werden. ${ }^{173}$ (Unverhältnismässig ist eine Datenbearbeitung dann, wenn kein vernünftiges Verhältnis zwischen Mittel und Zweck besteht.)

- Bundesorgane und Bundesaufgaben durchführende Organisationen dürfen nur Daten bearbeiten, die sie für ihren gesetzlichen Zweck wirklich benötigen, sei es, dass die Datenbearbeitung im betreffenden Bundesgesetz konkret beschrieben ist, oder dass sie sich aus den gesetzlichen Aufgaben klar ergibt. ${ }^{174}$

- Schliesslich klärt das DSG auch, wieweit der Patient beim praktizierenden Arzt Einsicht in seine Krankengeschichte oder eine Kopie derselben verlangen kann (vgl. Kap. 9.1.).

Praktisch alle Kantone haben für die Kantonsverwaltung und die Gemeinden und damit für die öffentlichen Spitäler und Heime - kantonale Datenschutzgesetze erlassen, die sich mehr oder weniger am DSG des Bundes orientieren.

\section{Elektronischer Datenaustausch}

Patientendaten dürfen zwischen Ärzten, Spitälern, anderen Behandelnden, aber auch Versicherungen nur geschützt ausgetauscht werden: E-Mails müssen verschlüsselt werden.

Wenn hingegen eine Patientin ihren Arzt mit einem unverschlüsselten E-Mail kontaktiert, darf der Arzt vom impliziten Einverständnis der Patientin ausgehen, auf demselben Kanal zu antworten. Eine andere Frage ist, ob er dies auch tun soll - hier ist im Einzelfall abzuwägen; zumindest sollte der Arzt sicher wissen, dass wenigstens die E-Mail-Adresse wirklich diejenige der Patientin ist.

\section{Künftiges Bundesgesetz über das elektronische Patientendossier (EPDG)}

Der Bund will in den nächsten Jahren ein Bundesgesetz über das elektronische Patientendossier (EPDG) erlassen. Damit wird nicht die elektronische Krankengeschichte des Arztes oder Spitals geregelt. Das elektronische Patientendossier soll vielmehr als sogenanntes «Sekundärsystem» über Praxen, Spitäler und weitere Einrichtungen hinweg für die weitere Behandlung und Mitbehandler relevante Ausschnitte aus dem «Primärsystem», d.h. der elektronischen Krankengeschichte in der Arztpraxis oder im Spital, verfügbar machen. Austrittsberichte, Röntgen- und Laborbefunde sollen jedem Arzt zugänglich sein, der vom Pati-

173 Art. 4 Abs. 1 DSG (revidierte Bestimmung in Kraft seit Januar 2008). Amédéo Wermelinger/ Daniel Schweri, Teilrevision des Eidgenössischen Datenschutzrechts - Es nützt nicht viel, schadet es etwas?, in: Jusletter 3, März 2008, Randziffer 10: "Selbstverständlich bestehen aber auch im Bereich des Datenschutzes Grenzen, welche die verletzte Person - selbst mit eigener Zustimmung - nicht überschreiten darf [im Sinne von Art. 27 ZGB]. So wäre eine zeitlich unbegrenzte Einwilligung in die Bearbeitung sämtlicher Personendaten einer Person sicher unzulässig.»

174 Art. 17 DSG. 
enten die Berechtigung erhalten hat. Ein wichtiges Element des künftigen EPDG ist die Unterscheidung der Gesundheitsfachpersonen in Berufsgruppen (Arzt, Physiotherapeut usw.) und Rollen (behandelnder Arzt, Arzt des Vertrauens, usw.). Deshalb sind Register wichtig, die alle in einer Funktion tätigen Gesundheitsfachpersonen aufführen (vgl. Kap. 2.2.).

\subsection{Meldepflichten und Melderechte}

Besteht eine gesetzliche Pflicht, einer Behörde eine Meldung zu machen, oder ist die Ärztin dazu berechtigt, so muss sie weder die Einwilligung des Patienten einholen noch durch die Gesundheitsdirektion von der ärztlichen Geheimhaltungspflicht entbunden werden. Meldepflichten und Melderechte gibt es sowohl auf Ebene des Bundes als auch auf jener der Kantone.

\section{Meldepflichten auf Bundesebene}

Besteht für den Arzt eine Meldepflicht, so muss er einen entsprechenden Vorfall melden. Unterlässt er die vorgeschriebene Meldung, könnte er sich gegebenenfalls sogar wegen Begünstigung strafbar machen.

- Übertragbare Krankheiten: Gemäss Epidemiengesetz melden «Ärzte, Spitäler sowie andere öffentliche oder private Institutionen des Gesundheitswesens [...] der zuständigen kantonalen Behörde übertragbare Krankheiten mit den Angaben, die zur Identifizierung erkrankter, infizierter oder exponierter Personen notwendig sind. Die kantonale Behörde leitet die Meldung dem Bundesamt für Gesundheit weiter.»(Art. 27 Abs. 1 Epidemiengesetz).

- Gesundheitsschädigungen mit möglichem Zusammenhang mit Militärdienst: «Kommt zwischen einer Gesundheitsschädigung und geleistetem Dienst ein Zusammenhang in Betracht, so ist der konsultierte Arzt [...] verpflichtet, den Fall sofort der Militärversicherung anzumelden. Er muss den Fall insbesondere anmelden, wenn der Patient oder seine Angehörigen es verlangen.» (Art. 84 Militärversicherungsgesetz, MVG).

- Schwangerschaftsabbruch «zu statistischen Zwecken der zuständigen Gesundheitsbehörde» melden, «wobei die Anonymität der betroffenen Frau gewährleistet wird und das Arztgeheimnis zu wahren ist.» (Art. 119 Abs. 4 in Verbindung mit Art. 120 Abs. 2 Strafgesetzbuch, StGB).

- Unerwünschte Wirkungen und Vorkommnisse im Zusammenhang mit Medikamenten und Medizinprodukten: Gemäss Heilmittelgesetz muss ein behandelnder Arzt «schwerwiegende oder bisher nicht bekannte unerwünschte Wirkungen und Vorkommnisse sowie Qualitätsmängel» im Zusammenhang mit der Anwendung von Heilmitteln melden (Art. 59 Abs. 3 HMG). Die Meldung erfolgt bei unerwünschten Wirkungen von Medikamenten an die regionalen Melde- 
stellen, bei Medizinprodukten an Swissmedic. Auch der klinische Prüfarzt muss im Rahmen der Forschung auftretende «schwerwiegende unerwünschte Arzneimittelwirkungen» Swissmedic und der Ethikkomission melden (Art. 22ff der Verordnung über klinische Versuche mit Heilmitteln, VKlin); im künftigen Humanforschungsgesetz gelten analoge Meldepflichten.

- Informationen, die der Versicherer benötigt, um seine Aufgabe zu erfüllen (Sozialversicherungsgesetze des Bundes): Die Sozialversicherungsgesetze des Bundes (KVG, UVG, MVG, IVG) enthalten eine Entbindung vom Patientengeheimnis. Allerdings ist diese immer auf jene Informationen begrenzt, «die der Versicherer benötigt», um seine Aufgabe zu erfüllen (vgl. Kap. 7.7. und 7.8.).

- Art. 22a KVG verpflichtet die Leistungserbringer zu Angaben ans BFS über «Anzahl und Struktur der Patientinnen und Patienten in anonymisierter Form». ${ }^{175}$

Die wichtigste Meldepflicht auf Kantonsebene:

der uaussergewöhnliche Todesfall»

Praktisch alle Kantone kennen eine Meldepflicht beim aussergewöhnlichen Todesfall: In der Schweiz ist gesetzlich vorgeschrieben, dass jeder Verstorbene von einem Arzt persönlich untersucht werden muss. Jeder Arzt muss daher in der Lage sein, eine Leichenschau sachgemäss durchzuführen. Nach heute gültiger rechtsmedizinischer Auffassung ist es unerlässlich, den Verstorbenen dazu vollständig zu entkleiden und sorgfältig von Kopf bis Fuss zu untersuchen - auch auf der Rückseite. Im Anschluss an die Leichenschau hat der Arzt den Tod formell zu bestätigen, indem er den Totenschein ausfüllt. Neben der Bestätigung, dass die betreffende Person sicher verstorben ist, muss der Arzt auch die Todeszeit möglichst genau angeben bzw. eingrenzen.

Schliesslich muss die Ärztin auf dem Totenschein deklarieren, ob es sich um einen natürlichen, einen nichtnatürlichen oder einen unklaren Todesfall handelt. Kommt sie nach korrekt durchgeführter Leichenschau zweifelsfrei zum Schluss, dass der Tod infolge einer «von innen kommenden», vorbestehenden Erkrankung eingetreten ist, so kann sie auf dem Totenschein einen natürlichen Tod bescheinigen, wobei sich der Begriff «natürlich» auf die Ursache des Todes bezieht.

175 Diese Angaben sollen gemäss Botschaft «ein Bild über die Tätigkeit der Leistungserbringer ermöglichen, [...] ohne dabei die Anonymität der Patientinnen und Patienten in Frage zu stellen» (BBI 2004 5573). Dies verbietet nach Auffassung der FMH personalisierte oder auch pseudonymisierte Angaben ans BFS. 
Dies hat zur Folge, dass der Leichnam zur Bestattung oder Kremation freigegeben wird und keine behördlichen Abklärungen vorgenommen werden. Kann kein natürlicher Tod attestiert werden, weil bei der Leichenschau sichere oder mögliche Hinweise auf einen nichtnatürlichen Tod gefunden werden oder der Tod plötzlich und unerwartet unter unklaren Umständen eingetreten ist, so handelt es sich um einen sogenannten aussergewöhnlichen Todesfall (AGT).

Aussergewöhnlich im rechtsmedizinischen Sinne sind alle Todesfälle, die plötzlich und unerwartet eingetreten sind, sowie alle gewaltsamen und solche, die vielleicht gewaltsam verursacht worden sein könnten. Neben den offensichtlich nichtnatürlichen Todesfällen (Tötungsdelikte, Suizide, Unfälle) gehören dazu auch alle plötzlichen und unerwarteten Todesfälle (inklusive des plötzlichen Kindstods), Todesfälle als sichere oder mögliche Folge diagnostischer oder therapeutischer Massnahmen sowie fund- und fäulnisveränderte Leichen unklarer Identität.

In den meisten Kantonen besteht bei AGTs eine gesetzliche Meldepflicht. Die Meldung muss in der Regel an die örtliche Polizei oder das entsprechende Untersuchungsrichteramt, in einigen Kantonen auch an den zuständigen Bezirks-, Kreis- oder Amtsarzt erfolgen. Dabei ist der Meldeerstatter automatisch von der ärztlichen Schweigepflicht entbunden (vgl. Kap. 7.1.).

Die Meldung eines AGT zieht weiterführende medizinische und polizeiliche Abklärungen nach sich. Die «normale» Leichenschau wird so zu einer amtlichen Leichenschau, einer sogenannten Legalinspektion.

Bleiben die Todesursache und insbesondere die Todesart auch nach dieser Legalinspektion unklar, bestehen Hinweise auf ein Fremdverschulden oder sollen Verletzungsmuster genau dokumentiert und interpretiert werden (z.B. im Rahmen der Unfallrekonstruktion), so wird der Leichnam von der Untersuchungsbehörde beschlagnahmt und einer rechtsmedizinischen Obduktion unterzogen. Falls nötig werden auch weitere labortechnische Untersuchungen durchgeführt.

Im Gegensatz zu einer klinisch-pathologischen Obduktion kann eine rechtsmedizinische Obduktion von Angehörigen nicht verweigert werden. Auch allfällige, von der verstorbenen Person zu Lebzeiten geäusserte oder schriftlich festgehaltene Verfügungen werden diesbezüglich hinfällig (vgl. Kap. 4.3.). Dabei gehört es zur ärztlichen Aufgabe, den Hinterbliebenen zu erklären, wieso welche weiteren Abklärungen notwendig sind. 
Dem Leichenschauarzt kommt also eine verantwortungsvolle Rolle zu: Aufgrund seiner Beurteilung der Todesart (natürlich, nichtnatürlich oder unklar) entscheidet er darüber, ob ein Todesfall überhaupt zur weiteren behördlichen Kenntnis gelangt und ob weitere Untersuchungen stattfinden. ${ }^{176}$ Daher ist eine Leichenschau als letzter Dienst an der Patientin zu verstehen und hat mit derselben Sorgfalt wie eine ärztliche Untersuchung zu Lebzeiten zu erfolgen.

\section{Die wichtigsten Melderechte auf Bundesebene}

Neben den Meldepfichten sind auch Melderechte gesetzlich verankert. Bei diesen kann ein Arzt die zuständigen Stellen bzw. Personen informieren; er muss dies aber nicht zwingend tun. Die nachfolgende Aufstellung enthält Melderechte sowohl an Behörden wie auch Auskunftsrechte an Personen.

- Mangelnde Fahrtüchtigkeit (Strassenverkehrsgesetz): Jeder Arzt kann Personen, die aus körperlichen oder geistigen Gründen oder wegen einer Suchterkrankung nicht mehr in der Lage sind ein Fahrzeug sicher zu lenken, der Aufsichtsbehörde für Ärzte sowie der für Erteilung und Entzug des Führerausweises zuständigen Behörde melden (Art. 15d Abs. 3 Strassenverkehrsgesetz, SVG). Die Meldung erfolgt an die Gesundheitsdirektion oder an das Strassenverkehrsamt.

- Strafbare Handlungen an Minderjährigen (Strafgesetzbuch): «Ist an einem Minderjährigen eine strafbare Handlung begangen worden, so sind die zur Wahrung des Amts- und Berufsgeheimnisses (Art. 320 und 321) verpflichteten Personen berechtigt, dies in seinem Interesse der Kindesschutzbehörde zu melden.» (Art. 364 Strafgesetzbuch, StGB).

- Betäubungsmittelmissbrauch (Bundesgesetz über die Betäubungsmittel): Fachleute im Gesundheitswesen können den zuständigen Behandlungs- und Sozialhilfestellen Fälle von vorliegenden oder drohenden suchtbedingten Störungen melden, wenn sie diese in ihrer amtlichen oder beruflichen Tätigkeit festgestellt haben, eine erhebliche Gefährdung des Betroffenen, seiner Angehörigen oder der Allgemeinheit vorliegt, und die meldende Person eine Betreuungsmassnahme als angezeigt erachtet (Art. 3c Betäubungsmittelgesetz, BetmG).

- Auskunft an Elternteile, die das Sorgerecht für ein Kind nicht haben (ZGB): Elternteile, die das Sorgerecht für ein Kind nicht haben, können «bei Drittpersonen, die an der Betreuung des Kindes beteiligt sind, wie namentlich bei Lehrkräften, Ärztinnen und Ärzten, in gleicher Weise wie der Inhaber der elterlichen Sorge Auskünfte über den Zustand und die Entwicklung des Kindes einholen». (Art. 275a Zivilgesetzbuch, ZGB). ${ }^{177}$

176 Die Leichenschaufunktion des praktizierenden Arztes stellt bereits eine Behördentätigkeit dar.

177 Sobald der Jugendliche urteilsfähig ist, hat sein Patientengeheimnis allerdings Vorrang. 


\section{Das wichtigste Melderecht auf kantonaler Ebene}

Die meisten Kantone kennen ein Melderecht, wenn der Arzt Hinweise auf ein Verbrechen oder schweres Vergehen feststellt.

\subsection{Allgemeines zu Arztzeugnis und Bericht}

Zeugnisse und Berichte werden von der behandelnden Ärztin ausgestellt.

Rechtlich besteht kein Unterschied zwischen einem Zeugnis und einem

Bericht. Arztzeugnisse und Berichte müssen wahrheitsgemäss erstellt werden. Wer falsche Zeugnisse oder Berichte schreibt, macht sich strafbar.

Ärztliche Zeugnisse, Berichte und Gutachten sind aus juristischer Sicht Urkunden. Rechtlich besteht kein Unterschied zwischen einem Zeugnis und einem Bericht. Die Standesordnung der FMH verlangt, dass Ärzte bei deren Ausstellung alle Sorgfalt anwenden und «nach bestem Wissen ihre ärztliche Überzeugung ausdrücken». Die Ausstellung von Gefälligkeitszeugnissen ist unzulässig. Zeugnisse und Berichte müssen transparent sein; dazu gehört, dass der Zweck der Schriftstücke, das Ausstellungsdatum und die Empfänger angegeben werden.

Zeugnisse und Berichte werden von der behandelnden Ärztin ausgestellt. Sie sollen transparent und für den Leser verständlich sein; zudem müssen sie wahr sein. Wenn beispielsweise die Arbeitsunfähigkeit im Zeugnis für den Taggeldversicherer und in demjenigen für die Arbeitslosenversicherung unterschiedlich angegeben wird, machen sich sowohl die Ärztin als auch der Patient verdächtig. Gemäss Strafgesetzbuch macht sich strafbar, wer vorsätzlich oder auch fahrlässig ein unwahres Zeugnis oder einen unwahren Bericht schreibt. ${ }^{178}$

Folgende Fragen müssen in einem Arztzeugnis oder Bericht einfach und verständlich beantwortet werden:

- Was hat die Ärztin selbst festgestellt?

- Wo muss sie sich auf Angaben des Patienten oder von Dritten stützen?

- Was ist ihre ärztliche Beurteilung?

\subsection{Berichte an mitbehandelnde Ärzte}

Das Patientengeheimnis gilt auch unter Ärzten wie auch gegenüber Angehörigen von anderen Gesundheitsberufen.

Eine Ärztin darf einem andern Arzt Angaben über eine Patientin nur weitergeben, wenn diese damit einverstanden ist. Zudem dürfen nur jene Informationen weitergegeben werden, die nötig sind. Zwar gelten auch hier rechtlich die ausdrückliche, die stillschweigende und auch die bloss mutmassliche Einwilligung 
der Patientin. Doch es ist sinnvoll, die Patientin ausdrücklich über die Abläufe zu informieren. Was für den Arzt selbstverständlich ist, ist es nicht für alle Patienten.

Der Hinweis «Ich melde Sie bei Dr. X zur Untersuchung an und schreibe ihr, was wir wissen und was wir von ihr erfahren möchten» benötigt nur wenig Gesprächszeit und kann verhindern, dass sich der Patient überrumpelt fühlt. Auch beim Spitalaustritt sollte eine Mitteilung erfolgen, im Sinne von: «Wir senden einen Bericht über die Spitalbehandlung und das weitere Vorgehen an die Ärzte $\mathrm{X}, \mathrm{Y}$ und Z; ist das in Ordnung für Sie?».

Im Spital darf man von der mutmasslichen Einwilligung des Patienten ausgehen, dass die an der Behandlung beteiligten Personen einander jene Patientendaten mitteilen dürfen und sollen, die für die sichere Behandlung nötig sind.

\subsection{Zeugnisse und Berichte an den Arbeitgeber und den Taggeldversicherer}

\section{Zeugnisse an Arbeitgeber oder Taggeldversicherer sollen transparent und wahr sein. Der Arbeitgeber hat keinen Anspruch, die Diagnose zu erfahren.}

\section{Bericht über berufliche Eintrittsuntersuchung}

Im Bericht über eine Eintrittsuntersuchung werden dem Arbeitgeber nur arbeitsmedizinische Schlussfolgerungen mitgeteilt. Er enthält Formulierungen wie «für die Arbeit als XY geeignet», «geeignet mit folgenden Einschränkungen...» oder «nicht geeignet». Der Bericht enthält keine Diagnosen.

\section{Arbeitsunfähigkeitszeugnis}

Arbeitsunfähigkeit ist die volle oder teilweise Unfähigkeit, im bisherigen Beruf oder Aufgabenbereich zumutbare Arbeit zu leisten. Sie kann durch eine körperliche, geistige oder psychische Beeinträchtigung der Gesundheit bedingt sein. ${ }^{179}$

Das Zeugnis hält fest, seit wann die Arbeitsunfähigkeit besteht, wie lange sie dauern wird und ob die Arbeitsunfähigkeit vollständig oder teilweise ist. Arbeitsunfähigkeitszeugnisse an den Arbeitgeber enthalten keine Diagnose, wohl aber die Angabe, ob die Behandlung wegen Krankheit oder Unfall erfolgte. 
Die Arbeitsunfähigkeit wird befristet. Wenn noch kein Enddatum angegeben werden kann, vermerkt der Arzt, dass die Arbeitsunfähigkeit «bis auf Weiteres» besteht und gibt gleichzeitig den nächsten Arzttermin an.

Wird die Patientin teilweise arbeitsunfähig geschrieben, bezieht sich eine Prozentangabe ohne weitere Information auf die Arbeitszeit (und nicht auf reduzierte Leistung bei voller Arbeitszeit). ${ }^{180}$ Das Zeugnis beschreibt gegebenenfalls die Tätigkeiten, die die Patientin ausführen kann bzw. nicht ausführen darf.

Dauert die Arbeitsunfähigkeit an, so muss die Ärztin beurteilen, welche anderen Tätigkeiten dem Versicherten zugemutet werden können.

Ist ein rückwirkendes Arbeitsunfähigkeitszeugnis nicht zu vermeiden, ist Transparenz besonders wichtig: Was hat der Arzt selbst festgestellt, was basiert auf Angabe des Patienten? Die Ärztin sollte keine rückdatierte Arbeitsunfähigkeit bestätigen, die nicht medizinisch plausibel ist.

Ist ein Elternteil zwar gesund, kann aber wegen Erkrankung des Kindes nicht zur Arbeit gehen, gilt dies für eine kurze Zeit - in der Regel bis drei Tage - als Arbeitsunfähigkeit. ${ }^{181}$ Entscheidend ist, wie rasch im konkreten Fall zumutbarerweise eine andere Betreuung gefunden werden kann (zur Begrenzung der Tätigkeiten bzw. zur Arbeitsunfähigkeitsfrage bei Jugendlichen und Schwangeren siehe Kap. 10.5.).

Einfache Arbeitsunfähigkeitszeugnisse für den Arbeitgeber sind gemäss TARMED grundsätzlich in der Konsultation inbegriffen.

Verschiedene kantonale Ärztegesellschaften haben mit den Sozialpartnern Zeugnisformulare und Vorgehensweisen vereinbart. Überzeugend erscheint insbesondere die Unterscheidung zwischen einfachen und detaillierten Zeugnissen. Benötigt ein Arbeitgeber ein detailliertes Zeugnis, stellt er dem Arzt ein schriftliches Anforderungsprofil der Stelle zu und übernimmt die Zeugniskosten.

\section{Zeugnisse und Berichte an den Taggeldversicherer}

Entscheidend für die Taggeldversicherung ist die Frage der Arbeitsunfähigkeit. Die meisten Taggeldversicherungen unterstehen dem Privatversicherungsrecht. Hier gehört es zur vertraglichen Treuepflicht des Arbeitnehmers gegenüber dem Arbeitgeber, dem Taggeldversicherer die nötigen Informationen für die Abklärung des Falls durch ein Arztzeugnis zukommen zu lassen. Untersteht eine Taggeldversicherung ausnahmsweise dem KVG, ist das Informationsrecht des Versicherers im Gesetz geregelt. ${ }^{182}$ 
Heikle Informationen sind an den beratenden Arzt des privaten Taggeldversicherers bzw. die Vertrauensärztin der Krankenkasse als Taggeldversicherer ${ }^{183} \mathrm{zu}$ richten. Die Privatversicherer haben keinen gesetzlich definierten Vertrauensarzt: Sogenannte Case-Manager sind keine Vertrauensärzte oder beratenden Ärzte. Aber die Grundsätze des Datenschutzgesetzes verlangen nach Auffassung der FMH, dass auch beim privaten Taggeldversicherer heikle medizinische Informationen, deren Bewertung ärztliches Wissen braucht, in verschlossenem Briefumschlag an den beratenden Arzt zur Beurteilung zugestellt werden können.

Für das Ausstellen von Zeugnissen und Berichten gibt es keinen vereinbarten Tarif - es gilt der freie Markt. Im Zweifelsfall ist der Tarif also vorher zu vereinbaren. Bei den seltenen Taggeldversicherungen nach KVG ist hingegen gemäss TARMED abzurechnen.

\subsection{Berichte und Abrechnung in der Krankenversicherung}

Die Krankenkasse muss die "notwendigen» Informationen erhalten. Zentral für den Datenschutz ist die Filterfunktion des Vertrauensarztes.

\section{Die notwendigen Informationen}

Alle Sozialversicherungsgesetze des Bundes (KVG, UVG, MVG, IVG) enthalten ein gesetzliches Informationsrecht des Sozialversicherers. Allerdings ist dieses Recht immer auf jene Informationen begrenzt, «die der Versicherer benötigt, um seine Aufgabe zu erfüllen». Dazu gehören «Angaben, um Leistungsansprüche zu beurteilen sowie Leistungen zu berechnen, zu gewähren und mit Leistungen anderer Sozialversicherungen zu koordinieren, ein Rückgriffsrecht gegenüber einem haftpflichtigen Dritten geltend zu machen oder Statistiken zu führen». ${ }^{184}$ Welche Informationen der Krankenversicherer tatsächlich benötigt, und wer sie innerhalb der Kasse bearbeiten darf, hängt also von der konkreten Fragestellung ab:

- Geht es um die Überprüfung der medizinischen Indikation der Behandlung, sollte die Vertrauensärztin entscheiden, was sie wissen muss. (Es ist nach Auffassung der FMH nicht sinnvoll, dass die Administration dem Arzt Fragen für einen Bericht an den Vertrauensarzt stellt, die aus Sicht des Vertrauensarztes unnötig sind.)

- Der Krankenversicherer darf gemäss Bundesgericht auch stichprobenweise Unterlagen an den Vertrauensarzt herausverlangen, im konkreten Gerichtsfall den Pflegebericht und die Vitalzeichenkontrolle des Pflegeheims. Gemäss

183 Urteil des Bundesgerichts K 121/03 vom 10. August 2004 E. 5.

184 Art. 84 KVG - die Aufzählungen in UVG, MVG, IVG sind ähnlich, aber nicht gleich. 
Eidg. Datenschutzbeauftragtem gilt dabei: «Die Versicherer [können] nur herausverlangen, was sie im Rahmen einer konkreten Überprüfung auch benötigen. Das heisst, es braucht die Absicht, bestimmte Fälle eingehender überprüfen zu wollen. Eine systematische Herausgabepflicht sämtlicher Diagnosen und zusätzlicher ärztlicher Informationen in allen Fällen im Sinne eines Automatismus wäre eine Datenbeschaffung auf Vorrat und damit unverhältnismässig. Denn mit dem Bundesgericht ist davon auszugehen, dass es angesichts der grossen Mengen von Abrechnungen den Versicherungen nicht möglich ist, jeden Einzelfall zu prüfen.» ${ }^{185}$

- Geht es um die Abklärung der Zuständigkeit der Kasse - beispielsweise gegenüber dem Unfall- oder Haftpflichtversicherer -, muss die Kasse hingegen praktisch dieselben Informationen erhalten wie ein UVG-Versicherer.

- Das Bundesgericht hat entschieden, dass die Vertrauensärztin auch Drittärzte zu einer fachlichen Stellungnahme auffordern kann. Sie braucht dazu - Ausnahmen vorbehalten - weder das Einverständnis des Versicherten noch dessen vorgängige Information. ${ }^{186}$

\section{Vertrauensarzt als Filter}

Die Ärztin oder der Arzt ist in begründeten Fällen berechtigt - und auf Verlangen der versicherten Person in jedem Fall verpflichtet -, medizinische Angaben nur dem Vertrauensarzt bekannt zu geben. Solche Angaben werden direkt an den vertrauensärztlichen Dienst der Kasse adressiert. Die Versicherung muss ihrerseits dafür sorgen, dass solche Schreiben intern korrekt weitergeleitet werden. Wo dieser Weg nicht funktioniert, wird die Ärztin sich überlegen, ob sie den Bericht dem Vertrauensarzt persönlich an dessen Praxisadresse zustellen lässt. ${ }^{187}$ Selbstverständlich müssen auch in den Fällen, in denen der Vertrauensarzt einer Behandlung vorgängig zustimmen muss, die Arztberichte direkt, also ohne Umweg über die Leistungsabteilung, zum vertrauensärztlichen Dienst gelangen.

«Vertrauensärzte und Vertrauensärztinnen geben den zuständigen Stellen der Versicherer nur diejenigen Angaben weiter, die notwendig sind, um über die Leistungspflicht zu entscheiden, die Vergütung festzusetzen oder eine Verfügung zu begründen. Dabei wahren sie die Persönlichkeitsrechte der Versicherten.» ${ }^{18}$

185 Website des Eidg. Datenschutz- und Öffentlichkeitsbeauftragten zum Urteil des Eidgenössischen Versicherungsgerichts vom 21. März 2007 K 12/06.

186 BGE 131 ॥| 413.

187 Das KVG kennt in Art. 57 den Vertrauensarzt als Person, nicht einen mehr oder weniger anonymen vertrauensärztichen Dienst.

188 Art. 57 Abs. 7 KVG. 
Nur der Vertrauensarzt in der Grundversicherung hat ein definiertes Qualifikationsprofil und eine gesetzlich garantierte Filterfunktion. Case-Manager der Krankenversicherer operieren dagegen im gesetzlich nicht definierten Rahmen. Der Versicherte kann deshalb auch nach früherer Zustimmung zu einem Case-Management jederzeit verlangen, dass heikle medizinische Informationen nur an die Vertrauensärztin gehen. Und nur die Vertrauensärztin kann die gemäss KVG vorgesehenen vorgängigen Zustimmungen zu Behandlungen erteilen, beispielsweise für Rehabilitationen. ${ }^{189}$

Die Krankenkassen bieten neben der Grundversicherung auch Privatversicherungen an. Nur für diese sind Gesundheitsfragebogen und Rückfragen bei den behandelnden Ärzten vor Abschluss der Versicherung zulässig.

Wenn im selben Leistungsfall beim gleichen Krankenversicherer die Grund- und die Zusatzversicherung betroffen sind, sollte der Vertrauensarzt auch in der Zusatzversicherung nach den Regeln des KVG arbeiten; die im KVG garantierte Filterfunktion der Vertrauensärztin soll nicht durch die private Zusatzversicherung bei derselben Kasse ausgehöhlt werden. ${ }^{190}$

Sozusagen als Notbremse für den Datenschutz kann der Patient auf die Rückerstattung der Kasse im konkreten Behandlungsfall verzichten. In diesem Fall geht das Patientengeheimnis dem gesetzlichen Informationsrecht der Kasse vor.

\section{Rechnungsstellung}

Der Patient muss eine detaillierte und verständliche Rechnung erhalten. Die Ärztin muss die Angaben machen, die nötig sind, damit der Patient überprüfen kann, wie die Vergütung berechnet wurde und ob die Leistung wirtschaftlich ist. Der Versicherer kann eine genaue Diagnose oder zusätzliche Auskünfte medizinischer Natur verlangen. Ob der Arzt die Versichertenkartennummer und die AHV-Nummer des Patienten wissen und auf der Rechnung angeben soll, muss der Patient selbst entscheiden können (siehe Kap. 7.1.).

Die Patientin sollte die Rechnung als Erste erhalten, diese kontrollieren und vor allem auch entscheiden, ob sie diese der Krankenkasse zur Rückerstattung zustellen will. Dies ist auch bei elektronischer Abrechnung machbar, wenn diese nicht direkt zur Krankenkasse geht, sondern zunächst bei einem Intermediär, dem sogenannten «Trust-Center», hinterlegt wird. Sobald der Patient die Rechnung an die Kasse weiterleitet, kann die Kasse sie im Trust-Center automatisch abfragen.

189 Rechtlich gesehen braucht es die Zustimmung des Vertrauensarztes auch in Managed-CareSystemen. Faktisch wird dies oft anders gehandhabt. (Die im Juni 2012 abgelehnte Managed-Care-Vorlage hätte die Möglichkeit legalisiert, den Care-Manager an Stelle des Vertrauensarztes einzusetzen.)

190 Dies ist die Auffassung sowohl der FMH wie der Schweiz. Gesellschaft der Vertrauensärzte. 
Die Spitäler, Apotheken, Labors und Heime hingegen rechnen in der Regel im «tiers payant» ab. Hier sieht systembedingt die Kasse die Rechnung zuerst; wenn der Patient dies nicht will, muss dies bereits bei Auftragserteilung mitgeteilt werden (siehe auch bei Kap. 7.1.). Zu wünschen ist, das der Arzt den Patienten vor Erteilung des Laborauftrags darüber informiert.

Der Arzt muss der Patientin für die von der obligatorischen Krankenpflegeversicherung übernommenen Leistungen und die anderen Leistungen zwei getrennte Rechnungen erstellen. ${ }^{191}$

\section{Datenweitergabe und Rechnungsstellung unter SwissDRG}

Beim DRG-Fallpauschalen-System für den akutstationären Bereich wird jeder Spitalaufenthalt anhand von bestimmten Kriterien wie Hauptdiagnose, Nebendiagnosen, Behandlungen, Schweregrad und Alter des Patienten einer Fallgruppe zugeordnet und pauschal vergütet. Es ist bzw. war grundsätzlich Aufgabe der Tarifpartner, die Modalitäten der Datenweitergabe bei der Rechnungsstellung vor der Einführung der DRG-Fallpauschalen zu vereinbaren. Eine Einigung kam jedoch nicht zustande, weshalb der Bundesrat diese per 1. Januar 2013 festlegte. Art. $59 \mathrm{KVV}$ hält neu in Abs. 1 lit. c fest, dass mit den Rechnungen nur Diagnosen und Prozeduren mitgeteilt werden dürfen, «die zur Berechnung des anwendbaren Tarifs notwendig sind». Gemäss Kommentar zur KVV bedeutet dies für DRG-Rechnungen, dass nur die gruppierungsrelevanten medizinischen Angaben an die Datenannahmestelle weiterzuleiten sind (und nicht alle Angaben, die ans Bundesamt für Statistik gehen). Das Eidg. Departement des Inneren EDI wird eine Ausführungsverordnung zur KVV-Revision vom 4. Juli 2012 erlassen.

Jeder Versicherer muss bis 31. Dezember 2013 über eine zertifizierte Datenannahmestelle verfügen (Art. 59a Abs. $6 \mathrm{KVV}$ ). Er kann diese selbst aufbauen oder die Funktion outsourcen. Die Spitäler leiten die Datensätze mit den administrativen und diejenigen mit den medizinischen Angaben gleichzeitig mit der Rechnung an diese Datenannahmestellen weiter (Art. 59a Abs. 3 KVV). Die Datenannahmestelle (die eigentlich eine Datenprüfstelle ist) bestimmt aufgrund einer elektronischen Plausibilisierung, «für welche Rechnungen eine weitere Prüfung benötigt wird». «Der Versicherer darf der Datensammelstelle keine Weisungen bezüglich der Datenweitergabe in Bezug auf einzelne Rechnungen erteilen.» Verlangt der Versicherer in der Folge weitergehende medizinische Auskünfte, muss er vorgängig den Versicherten fragen, ob die Angaben nur an den Vertrauensarzt oder an die Administration gehen dürfen. Bis die Versicherer zertifizierte Datensammelstellen haben, sind die medizinischen Daten ausschliesslich an den Vertrauensarzt zu übermitteln. 


\section{Offene Rechnungen}

Es kommt immer wieder vor, dass sich bei Ärzten offene Rechnungen von einzelnen Patienten häufen. Oft behandeln Ärzte Patienten, die finanzielle Probleme haben, bewusst weiter. Es empfiehlt sich, in dieser Situation (und mit dem Einverständnis des Patienten) mit der Fürsorge bzw. Sozialhilfe Kontakt aufzunehmen.

Daneben gibt es aber auch andere, nicht sozial begründete Fälle. Hier kann sich die Ärztin zumindest teilweise schützen, z.B. durch kürzere Intervalle bei der Fakturierung, Verweigern weiterer Behandlung bei offenen Rechnungen - ausser natürlich bei Notfällen - oder mit Bar- und Vorauszahlung. Bleiben Rechnungen unbezahlt, steht dem Arzt auch der Betreibungsweg offen.

Das KVG sieht seit dem 1. Januar 2012 keinen Leistungsaufschub der Krankenversicherer gegen säumige Versicherte mehr vor. ${ }^{192}$ Die Kantone können einen solchen jedoch weiterhin vorsehen. Die Krankenkassen müssen im Normalfall also die Rechnungen trotz Prämienschulden zahlen, ausser gegenüber Versicherten, die ihrer Prämienpflicht trotz Betreibung nicht nachkommen und vom Kanton auf einer «schwarzen Liste» ${ }^{193}$ erfasst werden. Diesen Personen sollen nur noch Notfallbehandlungen bezahlt werden. Wenn für die ausstehenden Prämien und Kostenbeteiligungen ein Verlustschein vorliegt, übernehmen die Kantone 85\% der Ausstände. Eine Pflicht, den Leistungsaufschub den Ärzten mitzuteilen, besteht zwar nicht. Diese sind jedoch nebst den Gemeinden und dem Kanton berechtigt, Einblick in die Liste zu nehmen. 


\section{Betreibungen}

Will der Arzt einen Fall dem Inkassobüro übergeben oder eine Patientin betreiben, braucht es nur wenig Angaben. Das Bundesgericht hatte in einem Fall entschieden, dass ein Anwalt, der im Inkassoauftrag oder im Betreibungsbegehren ausschliesslich die Personalien seines Klienten sowie als Forderungsgrund «Rechnung vom ...» und den Betrag angab, das Berufsgeheimnis nicht verletzte. Grundsätzlich müsste diese Rechtsprechung auch auf Ärztinnen angewendet werden. ${ }^{194}$ Das Berufsgeheimnis kann bereits dann verletzt werden, wenn bekannt gegeben wird, dass ein Behandlungsvertrag mit einem bestimmten Patienten besteht. Werden nur die oben beschriebenen Angaben gemacht, geht aus ihnen nicht hervor, ob die Forderung auf einem Behandlungsvertrag beruht. Wenn weitergehende Angaben wie «Behandlung vom ...» gemacht werden, wird das Berufsgeheimnis sicher verletzt. Der Arzt, der sich vom Patienten vorgängig schriftlich vom Berufsgeheimnis entbinden lässt, verletzt dieses nicht. Diese Einwilligung kann jedoch jederzeit zurückgezogen werden. Verschiedene Kantone sehen vor, dass die Ärztin zur Durchsetzung von Forderungen von Gesetzes wegen vom Berufsgeheimnis befreit ist. In den übrigen Kantonen herrscht eine uneinheitliche Praxis, was die behördliche Befreiung vom Berufsgeheimnis angeht. Weil der damit verbundene Aufwand gross, und es unproblematisch ist, eine Forderungseingabe mit minimalen Angaben $\mathrm{zu}$ machen, wird dieses Vorgehen wie oben beschrieben empfohlen. Allerdings darf nicht vergessen werden, dass die Entbindung vom Berufsgeheimnis im Laufe des Betreibungsverfahrens unerlässlich wird, weil dann präzisere Angaben zur Forderung gemacht werden müssen.

\section{Versäumte Konsultation}

Wenn ein Patient zur vereinbarten Konsultation nicht erscheint, stellt sich die Frage ob das Honorar geschuldet ist. Wenn der Patient objektiv verhindert und sein Ausbleiben somit unverschuldet war, muss der Arzt das Risiko tragen und kann nichts in Rechnung stellen. In jedem Fall muss der Arzt versuchen, den Schaden möglichst klein zu halten. Er muss die Zeit so gut wie möglich anders nutzen, kann vielleicht einen anderen Patienten vorziehen oder administrative Arbeiten erledigen. Rechtlich kommt es immer auf den konkreten Fall an. Fällt reservierte Untersuchungszeit in einem MRI aus, erscheint der Schaden offensichtlich - umgekehrt sieht es wohl bei einem Arzt aus, der eine sowieso überfüllte Sprechstunde hatte und letztlich froh ist, dass er nicht noch später nach Hause kommt. ${ }^{195}$ 
Der FMH-Rechtsdienst empfiehlt, den Patienten mit einem Hinweis im Wartezimmer oder auf den Aufgebotskärtchen eine klare Regelung betreffend versäumter Konsultationen oder verspäteter Abmeldungen zu kommunizieren; üblich ist eine 24-Stunden-Frist. Im Streitfall ist die Schadenersatzforderung dennoch nicht immer einfach durchzusetzen; zu prüfen bleibt in jedem Fall, ob der Patient unverschuldet nicht kam.

\subsection{Berichte und Abrechnung im UVG, MVG und IVG}

Der Arbeitgeber und die verunfallte Arbeitnehmerin müssen einen Unfall unverzüglich dem UVG-Versicherer melden. Besteht ein möglicher Zusammenhang mit einem geleisteten Militärdienst, muss der Arzt den Gesundheitsschaden der Militärversicherung melden. Bei der Invalidenversicherung besteht neu ein Melderecht ab 30 Tagen Arbeitsunfähigkeit im Hinblick auf die Früherfassung.

\section{Berichte und Abrechnung im UVG}

Wenn ein Arbeitgeber oder eine verunfallte Person einen Unfall melden, dann übergeben sie das Arztzeugnis-Formular aus der Bagatellunfall-Meldung oder die Unfallmeldung ihres UVG-Versicherers der behandelnden Ärztin. Diese füllt den medizinischen Teil aus und leitet die Unterlagen an den Versicherer weiter.

Im Unterschied zum KVG gibt es im Unfallversicherungsgesetz (UVG) keinen gesetzlich definierten Vertrauensarzt. Dennoch sollte der Arzt nach Auffassung der FMH in Ausnahmefällen medizinisch heikle Informationen, deren Beurteilung ärztliches Wissen benötigt, an den Kreisarzt der Suva bzw. an den beratenden Arzt des privaten UVG-Versicherers adressieren. Dieser soll eine Triage vornehmen und die Informationen soweit möglich filtern.

Auch gemäss UVG muss die Ärztin dem Versicherer eine detaillierte und verständliche Rechnung zustellen. Sie muss ebenfalls alle Angaben machen, die der Versicherer benötigt, um die Leistungsansprüche zu beurteilen. Weil der Unfallversicherer sowohl die Behandlung wie auch Taggelder und Renten bezahlt, und weil das UVG eine kausale Versicherung ist, benötigt der Unfallversicherer oft mehr Informationen als eine Krankenkasse.

Der Unfallversicherer bezahlt die Rechnung des Leistungserbringers direkt; für den Versicherten gibt es weder Selbstbehalt noch Franchise. 


\section{Berichte und Abrechnung im MVG}

Wenn ein Arzt eine Gesundheitsschädigung behandelt, die möglicherweise im Zusammenhang mit einem geleisteten Militärdienst steht, so muss er diese gemäss Militärversicherungsgesetz der Suva melden. Kommt ein Arzt dieser Meldepflicht nicht nach, haftet er für die Folgen.

Auch das MVG kennt keinen gesetzlich definierten Vertrauensarzt - es gilt sinngemäss dasselbe wie im UVG. Die Rechnung des Leistungserbringers wird von der Suva bezahlt; für den Versicherten gibt es weder Franchise noch Selbstbehalt.

\section{Berichte und Abrechnung im IVG196}

Seit Januar 2008 gibt es im Rahmen der 5. IV-Revision ab 30 Tagen Arbeitsunfähigkeit die Möglichkeit der sogenannten Früherkennung und von Frühintegrationsmassnahmen. Damit soll der Arbeitsplatzverlust verhindert werden. Zur Abklärung einer Frühintegration kann sich der Versicherte selbst melden; die Meldung kann aber auch durch Dritte, etwa den Arzt, die Arbeitgeberin oder den Taggeldversicherer erfolgen - auch gegen den Willen des Arbeitnehmers.

Die Versicherten müssen schon im Hinblick auf die Frühintegrationsmassnahmen die Ärzte nicht nur ermächtigen, Auskünfte zu erteilen, sondern auch «alle Unterlagen zur Verfügung stellen, die für die Abklärung im Rahmen der Früherfassung erforderlich sind». ${ }^{197}$

Von der Meldung zur Frühintegration zu unterscheiden ist die formelle Anmeldung für Integrationsmassnahmen, sobald die betroffene Person «von Invalidität bedroht» ist. Für Umschulungsmassnahmen reicht eine prognostizierte Erwerbsunfähigkeit von $20 \%$.

Es ist Aufgabe des RAD-Arztes, die verbleibende Arbeitsfähigkeit zuhanden der IV festzustellen.

Gemäss ATSG ist die Erwerbsunfähigkeit der ganze oder teilweise Verlust der Erwerbsmöglichkeiten auf dem in Betracht kommenden ausgeglichenen Arbeitsmarkt, wobei die Erwerbsunfähigkeit durch Beeinträchtigung der körperlichen, geistigen oder psychischen Gesundheit verursacht wird und nach zumutbarer Behandlung und Eingliederung verbleibt. ${ }^{198}$ Seit 2008 wurde das ATSG zudem um folgenden Absatz ergänzt: «Für die Beurteilung des Vorliegens einer Erwerbsunfähigkeit sind ausschliesslich die Folgen der gesundheitlichen Beeinträchtigung

196 Vgl. zur 5. IV-Revision die Beitrage von S. Ritler, 5. IVG-Revision: neue Instrumente fördern die Eingliederung, Schweiz. Ärztezeitung 8/2008; S. 310f, sowie Hanspeter Kuhn, Zuckerbrot und ..., Schweiz. Ärztezeitung 8/2008; S. 312-16.

197 Art. 3c Abs. 3 IVG.

198 Art. 7 Abs. 2 ATSG. 
zu berücksichtigen. Eine Erwerbsunfähigkeit liegt zudem nur vor, wenn sie aus objektiver Sicht nicht überwindbar ist.» Diese Änderung gilt für alle Sozialversicherungen, also auch für das UVG und das MVG.

Der Invaliditätsbegriff im ATSG gilt für alle Sozialversicherungsgesetze. ${ }^{199}$ Die Zumutbarkeit von Eingliederungsmassnahmen wurde mit der 5. IVG-Revision schärfer definiert: «Als zumutbar gilt jede Massnahme, die der Eingliederung der versicherten Person dient; ausgenommen sind Massnahmen, die ihrem Gesundheitszustand nicht angemessen sind.» $»^{200}$ Diese Änderung gilt nur im IVG, also nicht im UVG und im MVG.

Ebenfalls verschärft wurden die Kriterien für den Rentenanspruch. Anspruch auf eine Rente haben Versicherte, die ihre Erwerbsfähigkeit oder die Fähigkeit, sich im Aufgabenbereich zu betätigen, nicht durch zumutbare Eingliederungsmassnahmen wieder herstellen, erhalten oder verbessern können, während eines Jahres ohne wesentlichen Unterbruch durchschnittlich mindestens 40 Prozent arbeitsunfähig gewesen sind oder nach Ablauf dieses Jahres zu mindestens 40 Prozent invalid (Art. 8 ATSG) sind. ${ }^{201}$ Auch diese Änderung gilt nur im IVG.

Die Rechnungen für medizinische Eingliederungsmassnahmen wie auch für die Arztberichte werden direkt an die auftraggebende IV-Stelle gerichtet. Der Versicherte zahlt weder Franchise noch Selbstbehalt.

\subsection{Zeugnisse und Berichte an Pensionskassen und Lebensversicherungen}

Im überobligatorischen Bereich können die Pensionskassen Versicherungsvorbehalte machen. Sie müssen aber den bisherigen Vorsorgeschutz garantieren. Die Lebensversicherung ist eine Privatversicherung; sie basiert damit auf dem Prinzip der Vertragsfreiheit und der Informationssymmetrie.

\section{Pensionskassen}

Pensionskassen müssen das Freizügigkeitsgesetz in der beruflichen Vorsorge respektieren: Der bisher erworbene Vorsorgeschutz bleibt auch beim Stellenwechsel garantiert - auch der überobligatorische. Das bedeutet, dass trotz überobligatorischer Versicherung dann keine Gesundheitsfragen gestellt werden dürfen, wenn die versicherten Pensionskassenleistungen an der vorherigen Stelle gleich

201 Art. 28 IVG. Das Parlament wollte zwar explizit nicht die Rentenansprüche von psychisch Kranken verhindern (deren Zustand ja typischerweise lange oder immer instabil ist) - offen bleibt aber, weshalb dann die Definition überhaupt geändert wurde. 
gut waren wie an der neuen. ${ }^{202}$ Auch genetische Untersuchungen dürfen nicht verlangt werden. Der Versicherer darf auch nicht nach Ergebnissen früher durchgeführter genetischer Untersuchungen fragen (vgl. Kap. 5.1.).

Berichte an die Pensionskasse werden an den vertrauensärztlichen Dienst der Pensionskasse adressiert. ${ }^{203}$

\section{Lebensversicherungen}

Aus Effizienzgründen lässt der Lebensversicherer das Berichtsformular in der Regel durch die behandelnde Ärztin ausfüllen; er kann aber auch die Untersuchung und Berichterstattung durch einen bisher nicht an der Behandlung beteiligten Arzt veranlassen.

Das Bundesgesetz über genetische Untersuchungen beim Menschen (GUMG) begrenzt die Informationsrechte des Lebensversicherers im Bereich der genetischen Untersuchungen: Versicherungseinrichtungen dürfen als Voraussetzung für die Begründung eines Versicherungsverhältnisses weder präsymptomatische noch pränatale genetische Untersuchungen verlangen. ${ }^{204}$ Bei Versicherungssummen bis höchstens 400000 Franken dürfen sie auch nicht nach durchgeführten genetischen Untersuchungen fragen (vgl. Kap. 5.1.).

202 Art. 14 Freizügigkeitsgesetz in der beruflichen Vorsorge: «Der Vorsorgeschutz, der mit den eingebrachten Austrittsleistungen erworben wird, darf nicht durch einen neuen gesundheitlichen Vorbehalt geschmälert werden. Die bei der früheren Vorsorgeeinrichtung abgelaufene Zeit eines Vorbehalts ist auf die neue Vorbehaltsdauer anzurechnen. Für die Versicherten günstigere Bedingungen der neuen Vorsorgeeinrichtung gehen vor."

203 Art. 3 Freizügigkeitsverordnung (SR 831.425): «Bei Stellenwechsel dürfen medizinische Angaben (d.h. bestehende Vorbehalte und Vorbehaltsdauer) der bisherigen Vorsorgeeinrichtung nur von deren vertrauensärztlichem Dienst demjenigen der neuen Vorsorgeeinrichtung übermittelt werden. Dazu bedarf es überdies der Einwilligung des Versicherten.» Vgl. auch Anhang 4 zur FMH-Standesordnung (Richtlinie für arbeitsmedizinisch tätige Ärztinnen und Ärzte) von 1998.

204 Art. 26 GUMG. 


\subsection{Zeugnisse und Berichte im Asylverfahren}

Zeugnisse und Berichte im Asylbereich werden dann verlangt, wenn es um den Nachweis von Folter oder um die Frage geht, ob ein abgewiesener Asylbewerber aus medizinischen Gründen nicht ins Herkunftsland zurückgeschafft werden kann.

In Asylverfahren ist die Situation zwischen der Ärztin und der zuständigen Asylbehörde aus strukturellen Gründen oft nicht einfach: Das Bundesamt für Migration (BFM) und die Asylrekurskommission haben keinen vertrauensärztlichen Dienst, und sie geben selten Gutachten in Auftrag. In der Regel verfassen die behandelnden Ärzte entweder im Auftrag ihrer Patientin oder der Behörden Berichte, ${ }^{205}$ die dem Asylrichter bzw. dem BFM als einzige medizinische Entscheidgrundlage zur Verfügung stehen. Für Arztberichte im Asylverfahren gelten folgende Empfehlungen: ${ }^{206}$

- «Arztberichte im Asylverfahren sollen u. E. das Hauptgewicht darauf legen, aktuelle Befunde möglichst präzise und fachlich korrekt zu beschreiben. Abgeleitet aus dieser syndromalen Beschreibung der Befunde können entsprechende Diagnosen nachvollziehbar gestellt werden. Wenn Krankheiten diagnostiziert werden, sollen Angaben über die nötige Behandlung hinzugefügt werden. Die anamnestischen Angaben sind ohne Wertung und ohne weiteren Kommentar als Aussagen des Gesuchstellers wiederzugeben. Auf spekulative Äusserungen zu kausalen Verknüpfungen soll verzichtet werden. Allenfalls muss die nicht weiter abgestufte Aussage genügen, die erhobenen Befunde seien vereinbar mit den vorgebrachten anamnestischen Angaben.

- Falls eine posttraumatische Belastungsstörung vorliegt, soll dieses klar und deutlich festgestellt werden. Mit der Diagnosestellung ist automatisch ebenfalls ausgesagt, dass ein Trauma stattgefunden hat. Wie, wann und unter welchen Umständen dieses Trauma vorgefallen ist, stellt der behandelnde Arzt im Rahmen der Therapie mit für ihn genügender Sicherheit anhand des Inhalts von Intrusionen und Alpträumen problemlos fest. Dass dies für das BFM keine rechtsgenügende Beweiskraft haben kann, gehört zu den Realitäten des Asylverfahrens.»

Oftmals fordert die Asylbehörde die Asylbewerberin auf, Arztberichte auf eigene Kosten in Auftrag zu geben. Nach den allgemeinen Verwaltungsrechtsgrundsätzen müssten die Behörden medizinische Fragen von Amtes wegen abklären und auch die Kosten für die notwendigen Arztberichte übernehmen. Dies müssten aber die Asylbewerber vor dem Asylrichter durchfechten.

205 Das Berichtsformular kann von der Website des BFM heruntergeladen werden, www.bfm.admin.ch

206 Zit. nach: Thomas Maier, Arztberichte im Asylverfahren, SÄZ 6/2006; S. 225 - 29. 


\title{
Der Arzt als Gutachter
}

\subsection{Allgemeines zum ärztlichen Gutachten}

\author{
Ist der Arzt als Gutachter tätig, steht die Wahrheitsfindung im Vordergrund, \\ nicht die Therapie. Er vertritt damit nicht primär die Interessen des Patienten, \\ sondern er muss die gestellten Fragen unvoreingenommen nach bestem \\ Wissen und Gewissen beantworten.
}

Versicherungen sind zur Abklärung ihrer Leistungspflicht oft auf medizinisches Expertenwissen angewiesen, ebenso die Gerichte für die Beurteilung von Rechtsstreitigkeiten. Dabei geht es insbesondere um Fragen bezüglich des ursächlichen Zusammenhangs zwischen Unfallereignis und Gesundheitsschaden, um die konkrete Arbeitsunfähigkeit oder Erwerbsunfähigkeit des Patienten, um die Ermittlung des Integritätsschadens ${ }^{207}$ oder um die Frage, ob ein ärztlicher Behandlungsfehler begangen wurde und, falls ja, ob er einen Gesundheitsschaden verursacht hat.

Zwischen dem Besteller des Gutachtens und dem Gutachter besteht ein Auftragsverhältnis. Der Experte muss den Auftrag sorgfältig und persönlich ausführen. Letzteres ist nicht nur hinsichtlich der erwarteten Fachkompetenz bedeutend, sondern auch im Zusammenhang mit möglichen Ablehnungsgründen beim einvernehmlichen Gutachten. Der Gutachter hat Anspruch auf ein Honorar, das vorgängig vereinbart werden sollte. Auch der Gutachter übt den Arztberuf aus und benötigt nach Auffassung der FMH wie auch des Kantonsarztes und der Staatsanwaltschaft Bern eine Berufsausübungsbewilligung, wenn er in eigener Verantwortung tätig ist. ${ }^{208}$

Da der Gutachter für die Beurteilung der gestellten Fragen kompetent sein muss, drängt es sich unter Umständen auf, von Beginn weg ein Expertenteam aus verschiedenen Fachrichtungen einzusetzen. Möchte ein Gutachter aufgrund seiner vertieften Analyse weitere Fachpersonen beziehen, soll er dies mit dem Auftraggeber absprechen.

Der Gutachter muss unbefangen sein. Er darf deshalb mit Personen, die am Verfahren beteiligt sind, weder befreundet noch verfeindet oder verwandt sein. Auch darf er zuvor nicht in den Fall involviert gewesen sein, sei es als behandelnder Arzt oder als Gutachter. Sich zu kennen bedeutet noch nicht zwangsläufig den

207 Dauernde erhebliche Schädigung der körperlichen, geistigen oder psychischen Integrität.

208 Die Berner Staatsanwaltschaft büsste einen Gutachter, der ohne Berufsausübungsbewilligung in eigener Verantwortung eine MEDAS betrieben hatte, wegen Missachtung des Gesundheitsgesetzes (Der Bund, 4. Oktober 2012). 
Verlust der Unbefangenheit, kann aber den entsprechenden Anschein erwecken. Wer Gründe kennt, die bei einer Partei Zweifel an der Unbefangenheit wecken könnten, tut gut daran, die entsprechenden Zusammenhänge vor Annahme des Auftrages offenzulegen.

Der Gutachter bereitet sich aufgrund der Akten auf die zielgerichtete Untersuchung des Exploranden vor und nimmt sich auch für diese genügend Zeit. Er informiert den Exploranden darüber, dass er einen nichttherapeutischen Auftrag wahrnimmt. Erwartet wird, dass sich der Experte als Fachspezialist unvoreingenommen mit dem medizinischen Sachverhalt auseinandersetzt, diesen aufgrund seines Fachwissens und seiner Erfahrung beurteilt und die entsprechenden Schlussfolgerungen zieht.

Das Gutachten soll der Fall- bzw. der Streiterledigung dienen. Daher muss es klar, vollständig und schlüssig sein. Für den Beweiswert ist entscheidend, dass das Gutachten für die streitigen Belange umfassend ist, auf allseitigen Untersuchungen beruht, auch die geklagten Beschwerden berücksichtigt, in Kenntnis der Vorakten (Anamnese) abgegeben worden ist, in der Darlegung der medizinischen Zusammenhänge und in der Beurteilung der medizinischen Situation einleuchtet und dass die Schlussfolgerungen des Experten begründet sind. ${ }^{209}$

Trotz seiner grossen Verantwortung ist der medizinische Gutachter kein Richter. Wichtig ist, dass er sich an die relevante Terminologie hält und nicht vermeintlich gleichbedeutende Begriffe aus andern Gebieten - insbesondere rechtsspezifische Begriffe - in seine Schlussfolgerungen einbettet. So hat sich der Arzt wohl zur Arbeitsunfähigkeit, nicht jedoch zur Erwerbsunfähigkeit oder Invalidität zu äussern. Er macht auch nicht Aussagen zur adäquaten Kausalität oder zum Verschulden im Behandlungsfehlergutachten. Erwünscht ist auch eine allgemein verständliche Sprache, die von diskriminierenden Äusserungen frei ist.

Das Gutachten sollte klar strukturiert sein und angeben, auf welche Elemente sich der Gutachter stützt (Dokumente, Befragung, Bildgebung, Untersuchung, Fachliteratur usw.). Der Unterschied zwischen Tatsachen und Parteiaussagen muss zum Ausdruck kommen, etwa durch indirekte Rede. Wiederholungen oder $\mathrm{Zu}$ sammenfassungen am Schluss sollten vermieden werden: Oft sind sie nicht identisch formuliert, schaden der Übersichtlichkeit und sind Anlass für neue Differenzen zwischen den Parteien. 


\section{2. Ärztliche Gutachten für die Sozialversicherung}

Um abzuklären, ob eine Person berechtigt ist, Leistungen der Sozialversicherung, etwa der IV, zu erhalten, muss die Sozialversicherung Auskünfte einholen.

Die Sozialversicherer müssen den relevanten Sachverhalt von Amtes wegen abklären. Der Versicherte muss sich nötigenfalls untersuchen oder begutachten lassen. Tut er dies nicht, so kann der Versicherer aufgrund der Akten verfügen oder die Erhebungen einstellen und Nichteintreten beschliessen.

Braucht es ein Gutachten, gibt der Versicherer dem Versicherten den Namen der Gutachterin bekannt. Der Versicherte kann die Gutachterin aus triftigen Gründen ablehnen, und er kann Gegenvorschläge machen. Oft muss die Gutachterin zu Kausalitätsfragen Stellung nehmen. Am Beispiel der Unfallversicherung lässt sich zeigen, was damit gemeint ist: Zunächst wird gefragt, ob ein Schaden vorliegt - und ob er die natürliche Folge eines Unfalles ist (natürliche Kausalität); es muss geklärt werden, ob ohne diesen Umstand der Schaden nicht, nicht in der gleichen Weise oder nicht zur gleichen Zeit eingetreten wäre. ${ }^{210}$ Zusätzlich wird ein adäquater Kausalzusammenhang verlangt, um Leistungen der Unfallversicherung auszurichten. Es muss also geklärt werden, ob der Schaden nicht nur im Einzelfall, sondern auch im gewöhnlichen Lauf der Dinge eintreten würde. Der adäquate Kausalzusammenhang ist zwar eine Rechtsfrage, die nicht vom Gutachter zu entscheiden ist. Die Richterin ist aber zur Beurteilung auch auf ärztliche Angaben angewiesen. Wie üblich, erwartungsgemäss oder aber wie selten ist der beobachtete Verlauf aus medizinischer Sicht?

Im Sozialversicherungsrecht gilt generell der Beweisgrad der überwiegenden Wahrscheinlichkeit. Überwiegend wahrscheinlich ist ein bestimmter Sachverhalt, wenn der entsprechenden Überzeugung keine konkreten Einwände entgegenstehen. Soweit zwischen zwei oder mehreren Möglichkeiten zu entscheiden ist, gilt derjenige Sachverhalt als überwiegend wahrscheinlich, der sich am ehesten zugetragen hat.

\section{Interessenkonflikte zwischen Gutachter und Versicherung}

Interessenkonflikte können auch zwischen Arzt und Sozialversicherung auftreten. So hat das Bundesgericht 2011 festgestellt, dass die «wirtschaftliche Abhängigkeit der Gutachterstellen» [MEDAS] von der auftraggebenden IV-Stelle «die Fairness des Verfahrens gefährdet». Es hat das BSV aufgefordert, die Regeln für die Auftragserteilung der Gutachten zu ändern. ${ }^{211}$

210 Zit. nach: Ulrich Meyer, Die Zusammenarbeit von Richter und Arzt in der Sozialversicherung, SÄZ 26/1990; S.1090 - 94.

211 Urteil des Bundesgerichts (9C_243/2010) vom 28. Juni 2011. 
(2) 


\section{Rechenschaftspflicht und Verantwortlichkeit des Arztes}

\subsection{Rechenschaftspflicht}

Die Rechenschaftspflicht des Arztes ist Teil des Auftragsrechts.

Der Arzt ist gemäss Art. 400 Abs. 1 OR verpflichtet, dem Patienten umfassend Rechenschaft abzulegen. Ärzte in öffentlich-rechtlich organisierten Spitälern stehen gemäss ihren öffentlich-rechtlichen Dienstpflichten unter einer analogen Rechenschaftspflicht.

Die Rechenschaftsablage umfasst die Berichterstattung über den medizinischen Verlauf und über die Kosten der Behandlung. Die Rechenschaft ist nur in dem Umfang abzulegen, als der Patient sie verlangt.

Bei nichturteilsfähigen (z.B. dementen) Patienten hat der Arzt die Rechenschaft an die nächsten Angehörigen des Patienten zu erteilen, in erster Linie an den Ehegatten, in zweiter Linie an direkte Nachkommen, ausnahmsweise an andere Verwandte, Mitbewohner oder ständige Betreuer. Liegt eine Patientenverfügung oder eine Vollmacht vor, die diesen Fall abdeckt, dann ist die Rechenschaft an die vom Patienten benannten Bevollmächtigten zu erteilen. Bei Patienten im Kindesalter ist die Rechenschaft gegenüber den Erziehungsberechtigten zu erstatten, bei verbeiständeten Personen an den Beistand (siehe Kap. 3.).

Elternteile ohne Sorgerecht können bei Ärzten «in gleicher Weise wie der Inhaber der elterlichen Sorge Auskünfte über den Zustand und die Entwicklung des Kindes einholen». ${ }^{212}$ Der Arzt hat dabei auch die Rechte des Kindes oder Jugendlichen zu wahren. Wenn die Jugendliche eine Information vertraulich halten möchte und in dieser Frage urteilsfähig ist, so ist ihr Geheimhaltungswille gegenüber beiden Elternteilen zu beachten. 


\title{
9.2. Einsichts- und Kopierrechte des Patienten an der Krankengeschichte
}

\begin{abstract}
Das Datenschutzgesetz des Bundes (DSG) regelt die Modalitäten der Einsichtsund Kopierrechte des Patienten in der Arztpraxis und im Privatspital. Grundsätzlich hat der Patient den Anspruch auf eine Gratiskopie der ganzen Krankengeschichte. In öffentlichen Institutionen kann es Abweichungen geben.
\end{abstract}

\section{Einsichts- und Kopierrechte des Patienten in der Arztpraxis und im Privatspital}

Der Patient hat grundsätzlich den Anspruch auf eine Gratiskopie der ganzen Krankengeschichte. ${ }^{213}$ Es gibt die folgenden Ausnahmen:

- Die Ärztin muss in der Kopie Angaben abdecken, die von Dritten - vor allem von Angehörigen - stammen, solange diese nicht eingewilligt haben, dass der Patient die Information erhält. Die Patientin kann grundsätzlich auch Austrittsberichte und Überweisungsberichte herausverlangen, denn sie sind Teil der Krankengeschichte (KG) des Arztes, der sie erhalten hat. Es ist allerdings sinnvoll, die Patientin aufzufordern, solche Berichte direkt beim absendenden Arzt zu verlangen. Denn nur so kann der Arzt, der den Bericht geschrieben hat, auch prüfen, ob er sich auf fremdanamnestische Angaben stützte, die er gegenüber der Patientin geheim halten muss.

- Die Ärztin darf Angaben abdecken, an deren Geheimhaltung sie ein überwiegendes eigenes Interesse hat. Praktisch geht es dabei nur um Einträge, mit denen sie sich sozusagen «Luft» gemacht hat, und die eigentlich gar nicht in die KG gehörten.

Der Patient hat gemäss Datenschutzgesetz keinen Anspruch auf das Original, sondern auf eine Kopie. Ob der Arzt dem Patienten die Original-KG gegen Quittung herausgeben will, ist somit eher eine Stilfrage, doch muss er sicherstellen, dass eine allfällige Herausgabe des Originals keine Geheimnisse Dritter verletzt (siehe oben). Will der Arzt die Original-KG herausgeben und liegt die letzte Behandlung weniger als 10 Jahre zurück, ist ihm aus rechtlichen Gründen zu empfehlen, eine Kopie der KG für sich zu erstellen, weil er während 10 Jahren für allfällige Behandlungsfehler haftet und zudem die Beweislast für die Einwilligung des Patienten in Behandlungen trägt. ${ }^{214}$ 
Das DSG hält fest, dass die Kopien grundsätzlich gratis gemacht werden. Der Arzt in freier Praxis oder im Privatspital darf nur dann einen Unkostenbeitrag verlangen, wenn «die Auskunftserteilung mit einem besonders grossen Arbeitsaufwand verbunden ist» oder der Patient dieselben Daten in den letzten 12 Monaten schon einmal kopiert haben wollte. ${ }^{215}$ Zudem muss der Arzt in diesem Fall dem Patienten vorgängig mitteilen, wie viel er verlangen will.

Röntgenaufnahmen zu kopieren ist hingegen teuer. Die Standardempfehlung auch der Haftpflichtversicherer lautet deshalb, sie der Patientin im Original mit Verzeichnis und gegen Quittung zur Verfügung zu stellen. Immer öfter werden die Röntgeninformationen digital aufbewahrt und dem Patienten wird auf Wunsch ein Datenträger (CD) erstellt.

\section{Einsichts- und Kopierrecht des Patienten im öffentlichen Spital und Heim}

Die Kantone können die Einsichts- und Kopierfragen an der KG im öffentlichen Spital und Heim selbst regeln. So verlangen verschiedene kantonale Spitalgesetze, dass der Patient in jedem Fall für die Kopien bezahlt, und nicht nur bei hohem Aufwand.

Grundsätzliche Unterschiede zum Einsichts- und Kopierrecht darf es hingegen für die öffentlichen Spitäler und Heime kaum geben, weil die revidierte Bundesverfassung von 1999 für die ganze Schweiz den Grundsatz der informationellen Selbstbestimmung vorschreibt. ${ }^{216}$ Dieser verlangt Transparenz für Bürgerinnen und Bürger.

\section{Einsichts- und Kopierrechte in die KG von verstorbenen Patienten}

Das Patientengeheimnis ist grundsätzlich auch über den Tod hinaus zu wahren. Dies ergibt sich aus dem Berufsgeheimnis im Strafgesetzbuch; das Prinzip gilt auch für die öffentlichen Spitäler und Heime. Wenn Angehörige Kopien der Krankengeschichte der verstorbenen Patientin oder von Teilen davon erhalten möchten, muss sich der Arzt fragen, ob die Verstorbene damit einverstanden wäre. Kennt er die Familienverhältnisse gut, und geht er von der mutmasslichen Einwilligung der Verstorbenen aus, darf er die Krankengeschichte zur Verfügung stellen, soweit dies für die Beantwortung der Fragen der Angehörigen notwendig ist. Dabei sind für die Abklärung eines Behandlungsfehlers andere Informationen relevant als für die Beurteilung der Urteilsfähigkeit der Patientin zu einem bestimmten früheren Zeitpunkt. Kennt der Arzt die Familienverhältnisse nicht oder kann er nicht mit Sicherheit davon ausgehen, dass die verstorbene Patientin mit der Einsichtnahme einverstanden wäre, hat er sich von der zuständigen Behörde vom Berufsgeheimnis befreien zu lassen. Wenn die KG eines Verstorbenen heikle Informationen enthält, kann es sinnvoll sein, mit den Angehörigen oder 
mit dem Kantonsarzt festzulegen, welcher unbeteiligte Arzt die KG im Hinblick auf die konkrete Fragestellung absuchen soll; dieser wird in einer allfälligen Kopie an Angehörige die nichtrelevanten Informationen abdecken.

\subsection{Die Verantwortlichkeit des Arztes - Haftung im Zivil- und Strafrecht}

\section{Der Arzt ist gegenüber der Patientin verpflichtet, mit seinem Wissen und Können darauf hinzuwirken, die gewünschte gesundheitliche Verbesserung zu erreichen. Er haftet aber nicht dafür, dass die Behandlung erfolgreich ist und muss einen bestimmten Erfolg auch nicht garantieren.}

\section{Die Fehlerfrage im Zivilrecht}

Damit eine Ärztin haftpflichtrechtlich verantwortlich ist, müssen drei Bedingungen erfüllt sein:

1. Die Ärztin hat einen Fehler begangen.

2. Die Patientin hat einen Schaden erlitten.

3. Es gibt einen Kausalzusammenhang zwischen dem Fehler und dem Schaden.

Im Zivilverfahren ist also die Fehlerfrage zentral. Gemäss Rechtsprechung muss die Behandlung nach den Regeln der ärztlichen Kunst erfolgen. Die Ärztin hat Kranke stets fachgerecht zu behandeln. Zum Schutze ihres Lebens oder ihrer Gesundheit muss sie die gebotene und zumutbare Sorgfalt beachten. Die Ärztin haftet dabei nicht nur für grobe Verstösse, sondern hat grundsätzlich für jede Sorgfaltspflichtverletzung einzustehen. Eine Sorgfaltspflichtverletzung - ein Fehler - ist gegeben, wenn eine Diagnose, eine Therapie oder ein anderes ärztliches Vorgehen nach dem allgemeinen fachlichen Wissensstand nicht mehr als vertretbar erscheint und damit ausserhalb der ärztlichen Kunst steht.

Als Fehler gilt allgemein, wenn

- eine nicht indizierte Massnahme vorgenommen wurde;

- eine indizierte Massnahme nicht vorgeschlagen oder fehlerhaft durchgeführt wurde;

- eine Patientin falsch oder gar nicht instruiert wurde.

Hingegen bildet eine blosse Komplikation - auch wenn sie schwerwiegende Folgen hat - keinen Fehler.

Zur ärztlichen Sorgfalt gehört auch die Berücksichtigung der eigenen Grenzen. Die Ärztin muss wissen, wann sie ihre Patientin an einen Kollegen oder an ein anderes Spital zur weiteren Abklärung bzw. Behandlung überweisen muss. Für Be- 
handlungen, welche die eigene fachliche Kompetenz überschreiten, kann eine Haftung aus Übernahmeverschulden resultieren. Die Ärztin haftet auch für die von ihrem Personal begangenen Fehler. Sie ist dafür verantwortlich, ihr Personal so zu instruieren, dass es seine Arbeit korrekt durchführen kann.

Ob eine Sorgfaltspflichtverletzung, also ein Fehler, vorliegt, ist immer nach den Umständen des konkreten Einzelfalles zu beurteilen. Bei der Fehlerfrage geht es darum, ob die Ärztin in der damaligen Situation aufgrund der vorhandenen Informationen und diagnostischen bzw. therapeutischen Möglichkeiten einen vertretbaren Entscheid gefällt hat. Die Frage, was man rückblickend allenfalls hätte besser machen können, darf somit nicht als Massstab für die Sorgfalt dienen. Es obliegt dem Patienten, den Fehler zu beweisen.

Die ärztliche Tätigkeit ist stets mit Gefahren und Risiken verbunden. Eine Ärztin muss deshalb nicht für jene Gefahren und Risiken einstehen, die mit jeder ärztlichen Handlung und mit der Krankheit an sich einhergehen. Stehen für Diagnose und Therapie mehrere anerkannte Möglichkeiten zur Auswahl, so gehört es zum pflichtgemässen Ermessen der Ärztin, sich für eine davon zu entscheiden.

In haftpflichtrechtlichen Auseinandersetzungen zeigt sich häufig, wie wichtig eine korrekte, rechtzeitige Patientenaufklärung und deren Dokumentation sind. Im Gegensatz zum Beweis des Fehlers, trägt die Ärztin die Beweislast für die korrekte Aufklärung. Kann sie eine korrekte Aufklärung nicht beweisen, so haftet sie auch für Komplikationen, ohne dass ein Fehler vorliegt - es sei denn, sie kann nachweisen, dass der Patient auch bei gehöriger Aufklärung in den vorgenommenen Eingriff eingewilligt hätte (hypothetische Einwilligung).

Wenn ein Patient gegenüber der Ärztin vermutet, dass ein Diagnose- oder Behandlungsfehler unterlaufen ist, so vereinbart die Ärztin mit ihm am besten einen Termin ausserhalb der Sprechstundenzeit, um die Situation in Ruhe zu besprechen. Ein offenes Gespräch und eine gute Kommunikation zu diesem Zeitpunkt sind sehr wichtig. Der Spitalarzt wird mit seinem Vorgesetzten eine Standortbestimmung vornehmen, denn mögliche Behandlungsfehler mit Kostenfolgen sind immer auch Chefsache. Der FMH-Rechtsdienst empfiehlt, sofort ein Gedächtnisprotokoll der Behandlung zu verfassen.

Vermutet der Patient den Fehler und stellt er den Arzt deshalb zur Rede, so verlangt die ärztliche Rechenschaftspflicht nicht die freiwillige Anerkennung des Fehlers und der Haftungsfolgen. Die ärztliche Rechenschaftspflicht bezieht sich auf die Offenlegung von Fakten, d.h. des Behandlungsverlaufs, nicht auf deren wertende Beurteilung gemäss medizinischen und rechtlichen Standards. Die Anerkennung eines Fehlers enthält stets eine Wertung. Dazu ist der Arzt nicht verpflichtet und gemäss Versicherungspolice meist auch nicht berechtigt (ausser in ganz klaren Fällen). Hingegen darf er keine unwahren oder irreführenden Angaben zum Sachverhalt machen. Er darf nicht die Krankengeschichte nachträg- 
lich ändern, einzelne Belege daraus entfernen oder dem Patienten einen Teil der Krankengeschichte zustellen mit der Bemerkung, es handle sich um «die» (ganze) Krankengeschichte.

Ist die Patientin mit den Erklärungen nicht zufrieden und macht Schadenersatzansprüche geltend, so ist die Haftpflichtversicherung zu informieren, auch wenn der Arzt nicht von einem Fehler ausgeht. Hierfür ist eine Entbindung vom Arztgeheimnis nötig. ${ }^{217}$ Wer die Meldung an die Haftpflichtversicherung macht, gesteht damit noch nicht einen Fehler ein.

Die Ärztin kann dem Patienten allenfalls aufzeigen, an wen er sich für eine weitere Beratung oder Zweitmeinung wenden kann. Je nach Region gibt es auch gut funktionierende Patientenorganisationen, die erste Abklärungen vornehmen.

Bestätigt sich die Vermutung des Patienten über einen Behandlungsfehler, wird er in der Regel mit Hilfe einer spezialisierten Anwältin eine Lösung mit der Haftpflichtversicherung des Arztes bzw. des Spitals suchen. In klaren Fällen kann man den Fall direkt regeln; in unklaren Fällen wird ein medizinisches Gutachten nötig sein.

\section{Gutachterstelle der FMH}

Die FMH führt eine aussergerichtliche Gutachterstelle, die unter bestimmten Voraussetzungen Behandlungsfehlergutachten organisiert. Bedingung ist, dass eine gewisse Wahrscheinlichkeit besteht, dass der Patient durch einen Diagnose- oder Behandlungsfehler einen erheblichen Gesundheitsschaden erlitten hat, und dass er deswegen noch kein Gericht angerufen hat. Der Gutachter wird von der betroffenen Fachgesellschaft vorgeschlagen. Das Verfahren ist reglementiert und für die Parteien transparent. ${ }^{218}$ Die FMH hat diesbezüglich aber kein Monopol; die Parteien können stattdessen Gutachter direkt suchen und beauftragen. Nur selten müssen Behandlungsfehlerfragen von der staatlichen Richterin entschieden werden.

Es empfiehlt sich bei Gutachten, die nicht durch die FMH-Gutachterstelle erarbeitet werden, mit dem Auftraggeber einen Kostenrahmen zu vereinbaren. 


\section{Haftpflichtversicherung für Ärzte}

Das Medizinalberufegesetz verpflichtet Ärztinnen und Ärzte zum Abschluss einer Berufshaftpflichtversicherung oder zur Erbringung gleichwertiger Sicherheiten. Auch gemäss FMH-Standesordnung ist für eine hinreichende Versicherung gegen Ansprüche aus beruflicher Haftpflicht zu sorgen. Wer ohne Haftpflichtversicherung praktiziert, verstösst gegen seine Berufspflichten, riskiert seinen eigenen Ruin aufgrund eines Behandlungsfehlers und vereitelt allenfalls berechtigte Ansprüche seiner Patienten im Schadensfall.

Grundsätzlich sind alle Risiken, die in den Versicherungsbedingungen der Police nicht explizit ausgeschlossen sind, versichert. Bei einem Versicherungswechsel ist auf eine lückenlose Deckung und bei Praxisaufgabe auf eine genügende Nachdeckung zu achten. Eine Überprüfung des Versicherungsschutzes drängt sich auf bei Ausweitung des Tätigkeitsgebietes oder bei der Anstellung von Ärzten durch den Praxisinhaber. Dabei braucht es in der Regel eine zusätzliche Versicherung auch für Praxisassistenten; hingegen sind die Stellvertreterinnen in der Regel mit der normalen Police versichert. Es empfiehlt sich eine Anpassung der Versicherungspolice an die effektive ärztliche Tätigkeit, weil die Praxis zeigt, dass die Versicherungssumme oft $\mathrm{zu}$ tief ist. ${ }^{219}$

Die Haftpflichtversicherung deckt nicht nur berechtigte Schadenersatz- und Genugtuungsansprüche, sondern wehrt auch unberechtigte Ansprüche ab. Sie hat ein Interesse an einer möglichst frühzeitigen Information über geltend gemachte Schadenersatzansprüche; eine entsprechende Anzeigepflicht ist in der Regel vertraglich festgeschrieben.

\section{Der Arzt im Strafverfahren}

Wird gegen Ärzte im Zusammenhang mit der medizinischen Behandlung ein Strafverfahren eröffnet, geht es meistens um fahrlässige Tötung oder fahrlässige Körperverletzung. Solche Strafverfahren sind zum Glück selten, weil sie der betroffenen Patientin oder den Hinterbliebenen kaum Vorteile bringen. Der Patient kann das Strafverfahren nur beschränkt beeinflussen. Die Hürde für eine strafrechtliche Verurteilung von Ärzten ist denn auch deutlich höher als für die Zusprechung einer Entschädigung an die Patientin im Zivilprozess. Patienten wollen in der Regel Schadenersatz für die fehlerhafte Behandlung und nicht die Verurteilung des Arztes durch den Strafrichter.

Für die betroffene Ärztin ist ein Strafverfahren belastend, und es empfiehlt sich, für die Wahrung ihrer Interessen einen eigenen Anwalt auch dann beizuziehen, wenn das Spital selbst einen externen Anwalt beauftragt. Die Interessen von Arzt und Spital können im Rahmen eines Strafverfahrens unterschiedlich sein. 


\subsection{Der unzufriedene Patient}

\section{Wie kann eine Ärztin einen Patienten beraten, der mit einer Behandlung nicht zufrieden ist? Es gibt verschiedene Wege und Institutionen mit je spezifischen Zuständigkeiten und Vor- und Nachteilen.}

Jede kantonale Ärztegesellschaft hat eine Ombudsperson eingesetzt, die vermitteln kann.

Bei vermuteten Verstössen gegen das FMH-Standesrecht kann sich die Patientin an die zuständige Standeskommission wenden. Zuständig ist je nach Basismitgliedschaft des betroffenen Arztes die Standeskommission der kantonalen Ärztegesellschaft, des VSAO oder des VLSS. Die grösseren öffentlichen Spitäler führen zudem je eine eigene Stelle für Patientenbeschwerden.

Patienten können sich mit ihren Beschwerden auch an die Kantonsärztin bzw. die kantonale Gesundheitsdirektion wenden. Je nach Kanton gibt es besondere Schlichtungs- oder Untersuchungsgremien, an welche die Gesundheitsdirektion verweisen wird.

Bei gravierenden Verstössen, beispielsweise einer groben Missachtung des Patientengeheimnisses oder gar sexuellen Übergriffen, stellt sich für die Patientin die Frage, ob sie Strafanzeige machen soll. Sie kann sich zwar im Strafverfahren als Zivilklägerin einbringen, hat aber nur beschränkten Einfluss auf das Verfahren; sie ist sozusagen mehr Objekt als Subjekt des Strafprozesses. Erfahrene Patientenberater raten oft eher zur Anzeige gegenüber der Standeskommission und/oder der Gesundheitsdirektion (auch wenn die Patientin auch in diesem Fall keine formelle Parteistellung hat). ${ }^{220}$

\section{Der unzufriedene Patient will die Rechnung nicht bezahlen}

Es kommt vor, dass der Patient die Arztrechnung nicht bezahlen will, weil er mit dem Behandlungsergebnis nicht zufrieden ist. Rechtlich gesehen schuldet der Patient die Bezahlung auch dann, wenn der erwartete Behandlungserfolg nicht eintritt oder er andere Meinungsverschiedenheiten hat mit seinem Arzt. Den Patienten zu betreiben oder vor Zivilgericht einzuklagen ist zwar grundsätzlich möglich. Vorzuziehen ist ein Gespräch mit dem säumigen Patienten oder allenfalls die Vermittlung durch eine Ombudsperson der kantonalen Ärztegesellschaft. 


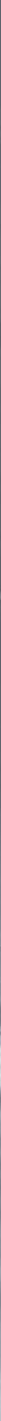




\section{Der Arzt als Arbeitnehmer oder Arbeitgeber}

\subsection{Anstellung am Spital oder in der Praxis}

Wer sich an einem Spital oder in einer Praxis anstellen lässt, muss sich im Betrieb einordnen. Er geniesst im Gegenzug den Schutz der Sozialversicherungen für Arbeitnehmer und braucht keine eigene Berufshaftpflichtversicherung abzuschliessen, solange er keine Patienten in eigenem Namen und auf eigene Rechnung behandelt.

\section{Einordnung in ein Unternehmen}

Das Spital haftet als Arbeitgeber und Leistungserbringer im Sinn des Sozialversicherungsrechts gegenüber seinen Vertragspartnern ${ }^{221}$ (Patienten und Lieferanten usw.) wie auch gegenüber Dritten ${ }^{222}$, vor allem den Angehörigen des Patienten. Es haftet für eine funktionierende und sichere Unternehmensorganisation, unter anderem für die sorgfältige Auswahl, Instruktion und Überwachung seiner Arbeitnehmer. Das bedeutet:

- Wer als Assistenzärztin oder Oberärztin im Spital arbeitet, muss sich sowohl fachlich wie organisatorisch den Vorgesetzten unterordnen. Dafür hat der Arbeitgeber Fürsorgepflichten gegenüber dem Arbeitnehmer. Assistenzärztin oder Oberärztin unterstehen dem Arbeitsgesetz, unter anderem betreffend Höchstarbeitszeiten.

- Der leitende Arzt und die Chefärztin arbeiten zwar fachlich weisungsungebunden; organisatorisch hingegen müssen auch sie sich dem Unternehmen unterordnen.

\section{Zivilrechtliche Haftung des Unternehmens für seine Angestellten}

Primär haftet das Unternehmen, also ein Spital oder eine Gruppenpraxis, für Schadenersatz und Genugtuung für allfällige Behandlungsfehler von Mitarbeitenden. Dennoch ist es für die angestellte Ärztin wichtig, darauf zu achten, dass die Unternehmensleitung die Haftpflichtfälle kompetent und konstruktiv führt. Wird nämlich grundlos Schadenersatz bezahlt, leidet das berufliche Ansehen der angestellten Ärztin unnötig. Wird umgekehrt trotz unterlaufenem Behandlungsfehler und daraus resultierendem Schaden ein Schadenersatz verweigert, riskiert die Ärztin als «Retourkutsche» des frustrierten Patienten eine Strafanzeige. 


\section{Strafrechtliche Haftung jeder Person für eigenes Versagen}

Bei Strafuntersuchungen gilt hingegen das Prinzip, dass jeder für seine eigenen Fehler einstehen muss. Auch der angestellte Arzt braucht dann eine eigene Strafverteidigerin. Das Spital sollte nach Auffassung der FMH mindestens einen Teil der Verteidigungskosten übernehmen. Der VSAO bietet zudem die Möglichkeit an, einer Kollektiv-Rechtsschutzversicherung beizutreten, die unter anderem die Kosten der Strafverteidigung deckt.

\section{Disziplinarische bzw. verwaltungsrechtliche Haftung am öffentlichen Spital}

Das öffentliche Spital ist Teil des Gemeinwesens. Wenn hier Unregelmässigkeiten oder Fehler auftreten, kann es angezeigt sein, dass Kanton bzw. Gemeinde als Träger eine disziplinarische oder eine verwaltungsrechtliche Untersuchung gegen Mitarbeitende und/oder die Institution eröffnen zur Abklärung der Frage, ob Verhaltenspflichten verletzt wurden oder ob die Institution nicht funktioniert hat.

\section{Standesrechtliche Verantwortlichkeit für alle FMH-Mitglieder}

Die FMH-Standesordnung gilt auch für angestellte Ärzte (siehe Kap. 2.4.). Sie verlangt, dass die Patientin weiss, mit wem sie den Behandlungsvertrag abschliesst: Ist sie stationäre Patientin bzw. Poliklinikpatientin des Spitals? Oder schliesst sie im Rahmen der Chefarztsprechstunde einen Behandlungsvertrag mit dem Arzt ab? Auch angestellte Ärzte, insbesondere in Cheffunktion, sollen sich deshalb dafür einsetzen, dass die Telefonbucheinträge, die Informationstafeln im Spital und die Anschriften der Kliniken und Räume sowie die Angaben auf den Patientenrechnungen übereinstimmen.

Die Standesordnung verlangt zudem, dass die Kontinuität der Betreuung auch bei angestellten Ärzten soweit als möglich sichergestellt wird..$^{223}$

\section{Sozialversicherungen und Steuern}

Wer angestellt ist, wird automatisch in einer Reihe von Sozialversicherungen versichert. Dazu gehören die Alters- und Hinterlassenenversicherung, die berufliche Vorsorgeversicherung, die (berufliche) Unfallversicherung und die Arbeitslosenversicherung. Zudem erhält der Angestellte bei Arbeitsunfähigkeiten und bei Abwesenheiten wegen Fortbildung, Militärdienst usw. den Lohn für eine bestimmte Zeit weiterbezahlt. 
Weil die Taggeldversicherung für den Krankheitsfall in der Schweiz nicht obligatorisch ist, soll beim Anstellungsgespräch geklärt werden, ob der Arbeitgeber für seine Angestellten eine Taggeldversicherung abschliesst. Wenn er das Lohnfortzahlungsrisiko selbst abdeckt, dauert seine Lohnfortzahlungspflicht nur wenige Wochen, während die Taggeldversicherungen eine längere Arbeitsunfähigkeit übernehmen (traditionell bis 720 Tage), um das finanzielle Risiko einer Erwerbsunfähigkeit bis zu einer allfälligen IV-Rente finanziell abzusichern.

Wer angestellt ist, hat weniger Spielraum für Steuerplanung: Er kann Geschäftsauto und Geschäftsessen grundsätzlich nicht an den Steuern abziehen, er zahlt die Steuern am Wohnort, und er kann die Steuerbelastung seines Einkommens auch nicht mit gestaffelten Investitionen ins Geschäft und überlegter Abschreibung des Goodwills zwischen verschiedenen Jahren ausgleichen. Möglich sind immerhin Pauschalspesenregelungen, die der Arbeitgeber mit der Steuerverwaltung aushandeln kann, und die insbesondere für Kaderangestellte einen gewissen Spielraum schaffen. Nicht abziehbar sind die Kosten für die Weiterbildung bis zum Facharzt. Nach Ansicht des Bundesgerichts ist der Arzt in Ausbildung, bis er die Facharztweiterbildung abgeschlossen hat. ${ }^{224}$

\section{Korruptionsstrafrecht im Spital oder Heim}

In öffentlichen Institutionen gilt das revidierte Korruptionsstrafrecht im Strafgesetzbuch: Ein Staatsangestellter macht sich strafbar, wenn er «im Zusammenhang mit seiner amtlichen Tätigkeit für eine pflichtwidrige Tätigkeit oder eine im Ermessen stehende Handlung oder Unterlassung für sich oder einen Dritten einen nicht gebührenden Vorteil» fordert, sich versprechen lässt oder annimmt. ${ }^{225}$ Für Belegärzte an Privatkliniken schliesslich können die Bestimmungen des UWG gegen die Privatkorruption relevant sein.

In öffentlichen Spitälern und Heimen müssen deshalb Forschungsverträge und Sponsoring der Industrie für die Durchführung und die Teilnahme an Kongressen intern offengelegt und je nach Reglement von einem Gremium genehmigt werden. Dasselbe gilt für den Belegarzt oder die Heimärztin an privaten Institutionen, wenn sie die Einkaufspolitik der Institution für Arzneimittel mitsteuern. ${ }^{226}$

224 Bundesgerichtsurteil vom 17. Oktober 2005, 2A.182/2005: « [...] Berufsaufstiegskosten, sind Investitionen in die Zukunft, ergänzen die Grundausbildung und gelten nicht als abzugsfähige Weiterbildungskosten".

225 Art. 322ter StGB.

226 Art. 4a Abs. 1 lit. b. UWG, vgl. auch Urteil des Bundesgerichts (2C_269/2011) vom 22. September 2011. Im Kanton Zürich können die mit dem Beruf zusammenhängenden Weiterbildungskosten, die einen Aufstieg in eine gehobenere Stellung im angestammten Beruf erlauben, vom steuerbaren Einkommen abgezogen werden, § 26 Abs. 1 lit. d StG. 


\title{
10.2. Der selbstständig tätige Arzt: Praxiseröffnung, Praxisübernahme und Praxisschliessung
}

\author{
Vor der Eröffnung oder Übernahme einer Praxis muss die Praxisbewilligung \\ sichergestellt sein. In einem Übernahmevertrag werden die Bedingungen \\ für die Übernahme festgelegt. Zudem muss der Umgang mit den Krankenge- \\ schichten geregelt werden.
}

\section{Berufsausübungsbewilligung und Behandlung von KVG-Patienten}

Voraussetzung für den Betrieb einer ärztlichen Praxis ist die Berufsausübungsbewilligung (BAB). Sie wird umgangssprachlich auch Praxisbewilligung genannt und ist die gesundheitspolizeiliche Bewilligung der kantonalen Gesundheitsbehörde, die den Arzt berechtigt, selbstständig als Arzt tätig zu sein und damit eine Praxis zu führen. Eine Berufsausübung braucht, wer Patienten in eigener medizinischer Verantwortung behandelt oder begutachtet (vgl. zu Letzterem Kap. 8.1.). Die Voraussetzungen für die Erteilung einer BAB für selbstständig tätige Ärzte sind auf Bundesebene im Medizinalberufegesetz (MedBG) festgehalten.227 Die Umsetzung erfolgt aber weiterhin durch die Kantone. Es ist eine Revision des MedBG geplant, wonach auch in der Praxis angestellte, aber fachlich eigenverantwortlich tätige Ärzte aufgrund des MedBG eine BAB bräuchten. Momentan ist es noch Sache der Kantone zu regeln, ob es eine BAB für in Arztpraxen angestellte Ärztinnen braucht.

Ende 2011 ist der Zulassungsstopp ausgelaufen, d.h., wer zulasten der OKP abrechnen möchte, braucht seit dem 1. Januar 2012 keine KVG-Zulassung mehr. Doch hat der Bundesrat im November 2012 dem Parlament vorgeschlagen, den Zulassungsstopp in der zuletzt gültigen Form per 1. April 2013 wieder für 3 Jahre einzuführen. Voraussichtlich wird das Parlament in der Frühjahrssession 2013 darüber entscheiden.

Zudem muss gemäss dem KVG-Rahmenvertrag bei santésuisse ${ }^{228}$ die ZSR-Nummer (ZSR = Zahlstellenregister) verlangt werden. Es handelt sich dabei zwar bloss um eine technische Abrechnungsnummer. Gemäss Bundesgericht nimmt jedoch die santésuisse mit der Nummernvergabe, wenn auch ohne gesetzliche Ermächtigung, eine den Versicherern kraft öffentlichen Rechts obliegende Pflicht wahr, nämlich die Prüfung der Voraussetzungen der Zulassung eines Leistungserbringers zur OKP. Deshalb hat das Bundesgericht das Schiedsgericht als zuständig erklärt für die Beurteilung von Streitigkeiten zwischen der santésuisse und einem Leistungserbringer betreffend Verweigerung einer ZSR-Nummer. ${ }^{229}$ Für die Ab-

228 Im Auftrag von santésuisse ist die SASIS AG (Tochterfirma der santésuisse) schweizweit für die Vergabe der ZSR-Nummern zuständig.

229 BGE 132 V 303. 
rechnung mit der IV erteilt die Zentrale Ausgleichsstelle ZAS zusätzlich eine sogenannte NIF-Nummer (numéro d'identification fournisseur). Die Zahlungsadresse ist mit dieser verbunden. ${ }^{230}$

\section{Praxisübernahme ${ }^{231}$}

Bei einer Praxisübernahme wird ein Übernahmevertrag abgeschlossen. Dazu muss zunächst der Vertragsgegenstand klar definiert werden, d.h., es muss beiden Vertragsparteien klar sein, was gekauft werden soll. Um dies zu bestimmen, sollte der Praxisinhaber ein Inventar erstellen, das Praxiseinrichtungen, Geräte und Medikamente umfasst.

Für die Festlegung des Kaufpreises kann grob zwischen der Bewertung des Inventars und des sogenannten Goodwills unterschieden werden. Während die Inventarwertschätzung nach objektiven Kriterien erfolgen kann, gibt es für den Goodwill keine Expertenlösung. Hier spielt der Markt. Eine transparente Expertenbewertung kann aber den Parteien als Diskussionsgrundlage dienen und einen Vertragsabschluss erleichtern.

Die Arbeitsverträge mit dem bisherigen Praxispersonal laufen grundsätzlich weiter. ${ }^{232}$ Will die Nachfolgerin das bisherige Personal nicht übernehmen, so muss der Verkäufer unter Einhaltung der vertraglichen Kündigungsfristen die Arbeitsverträge vor der Übergabe kündigen. Lehnt das Personal die Weiterbeschäftigung unter dem Nachfolger ab, so kann es das Arbeitsverhältnis unter Einhaltung der gesetzlichen Fristen kündigen. Übernimmt die Nachfolgerin das Personal, so sollte sie prüfen, ob keine Lohnausstände aus der Zeit vor der Übergabe bestehen. Dafür haftet sie nämlich solidarisch mit dem bisherigen Inhaber.

Bei gemieteten Praxisräumlichkeiten ist für die Übertragung des Mietverhältnisses die schriftliche Zustimmung des Vermieters notwendig. Dieser kann aber die Zustimmung nur aus wichtigen Gründen verweigern. ${ }^{233}$

230 www.zas.admin.ch/cdc/cnc3/cdc.php?pagid=33\&elid=391 Nicht geklärt ist, wie sich all diese Nummern mit dem Bundesgesetz über die Unternehmens-Identifikationsnummer (UIDG) vom 18. Juni 2010 vertragen sollen. Gemäss Art. 5 UIDG dürften Arzt und Spital sich auch gegenüber den Sozialversicherern durch blosse Angabe ihrer UID identifizieren.

231 Für die Praxiseröffnung oder die Übernahme einer Praxis stellt die FMH ihren Mitgliedern eine Reihe von Musterverträgen unentgeltlich zur Verfügung. www.fmh.ch $\rightarrow$ Service $\rightarrow$ Recht $\rightarrow$ Musterverträge.

232 Art. 333 OR.

233 Art. 263 OR. 
Bei einer Praxisübergabe endet der Behandlungsvertrag der bisherigen Ärztin. Der Nachfolger muss die Vertragsbeziehung mit den Patienten neu eingehen. Er darf deshalb die Krankengeschichten (KG), die er vom Verkäufer übernimmt, nur mit dem Einverständnis des Patienten einsehen. ${ }^{234}$ Transparenz und Sicherheit bietet das sogenannte «2-Schränke-Prinzip». In den ersten Schrank kommen die KGs von Patienten, die in der einen oder anderen Form der Übergabe ihrer KG zugestimmt haben. In der Regel erfolgt dies, indem die Patienten beim Nachfolger einen Behandlungstermin vereinbaren. Im zweiten Schrank bleiben jene KGs von Patienten, die noch nicht eingewilligt haben. Bei elektronischer KG-Führung gelten grundsätzlich die gleichen Regeln. Der Nachfolger muss die Daten(träger) während der gesetzlichen Aufbewahrungsfrist so sichern, dass sie nicht beschädigt werden und dass kein unbefugter Dritter Zugang darauf hat.

\section{Praxis als juristische Person}

Mit der Zunahme von Gruppenpraxen steigt das Bedürfnis nach komplexeren Organisationsformen, was mit ein Grund dafür sein dürfte, dass die Frage der Organisation von Arztpraxen als juristische Personen (primär GmbH und AG) immer aktueller wird.

Mit dem KVG wurde im Bundesrecht verankert, dass Ärztinnen und Ärzte HMOPraxen und Einrichtungen für die ambulante Krankenpflege betreiben können. ${ }^{235}$ Art. 36a KVG gilt auch für juristische Personen, die Ärzte anstellen, ohne als HMO-Praxis organisiert zu sein. ${ }^{236}$ Auch wenn das KVG juristische Personen zur Leistungserbringung zulasten der OKP grundsätzlich zulässt, liegt mindestens bisher $^{237}$ die Kompetenz darüber, ob eine Arztpraxis von einer juristischen Person betrieben werden darf, bei den für das Gesundheitswesen zuständigen Kantonen. ${ }^{238}$ Nicht alle, aber dennoch die Mehrheit der Kantone, lassen heute die Leistungserbringung in Form einer Kapitalgesellschaft (Aktiengesellschaft oder Gesellschaft mit beschränkter Haftung) zu. Die Vor- und Nachteile der möglichen Rechtsformen für die Praxis sollten im Voraus sorgfältig abgewogen bzw. geklärt werden. Dabei spielen verschiedene Einflussfaktoren eine Rolle: Anzahl Ärzte, (kantonale) rechtliche Möglichkeiten und Voraussetzungen, persönliche Wünsche und Ziele, Steueraspekte, Versicherung und Vorsorge usw.. Die FMH Services verfügen über ein Netz von Beratern in den Bereichen Treuhand, Finanzierung, Versicherungen und Recht.

234 Mit den Krankengeschichten (KGs) übernimmt der neue Praxisinhaber auch die Aufbewahrungspflicht für die KGs.

235 Art. 36 und Art. 36a KVG.

236 Urteil 9C_701/2008 vom 20. April 2009, Erw. 4.3.

237 Das Bundesgericht hat kürzlich die Anwalts-Aktiengesellschaft für alle Kantone zugelassen (Urteil vom 7. September 2012, 2C_237/2011). Es ist denkbar, dass mit analoger Begründung auch ähnlich organisierte Arztpraxen als $A G$ in allen Kantonen zugelassen werden könnten.

238 Urteil des Bundesgerichts 2P.231/2006 vom 10. Januar 2007, Erw. 5.4. 


\subsection{Praxisführung}

Auch wenn dies für viele Ärzte bei ihrer täglichen Arbeit nicht im Vordergrund steht, ist ein Arzt in der Praxis immer auch Unternehmer.

\section{Freie Arztwahl und Werbung}

Die Patientinnen haben grundsätzlich die freie Arztwahl und können jederzeit ihren behandelnden Arzt wechseln. Ausnahmen von dieser Regel bilden Versicherungsmodelle mit eingeschränkter Arztwahl (vgl. Kap. 4.12.).

Um sich in diesem Markt möglichst gut zu positionieren, möchten Ärztinnen vermehrt mit Werbung auf sich aufmerksam machen (vgl. Kap. 2.4.).

\section{Stellvertretung 239}

Massgebend für die Anforderungen an Praxisstellvertreter ist grundsätzlich das kantonale Recht. Die Gesundheitsdirektorenkonferenz empfiehlt den Kantonen folgende Regelungen, die auf einem gemeinsamen Vorschlag der FMH und Vertretern der Kantonsärzte beruhen:

- Jede Stellvertreterin soll in der Regel mindestens über ein anerkanntes Arztdiplom verfügen. Ausnahmen bilden hochqualifizierte ausländische Ärzte, die mangels Staatsvertrag ihr Diplom nicht anerkennen lassen können.

- Wer drei Jahre klinische Weiterbildung hat, kann in der Regel ohne Supervision eine Stellvertretung führen. Bei kürzer dauernder klinischer Weiterbildung scheint die Auflage einer sichergestellten Supervision angezeigt.

- Für Stellvertretungen soll kein Weiterbildungstitel verlangt werden. 


\subsection{Pflichten als Arbeitgeber}

\section{Stellt ein Arzt weitere Personen in seiner Praxis ein, ist er Arbeitgeber und hat seinen Angestellten gegenüber eine Reihe von Pflichten zu erfüllen.}

In der Regel und mit Vorteil werden alle wichtigen Punkte eines Anstellungsverhältnisses in einem schriftlichen Arbeitsvertrag geregelt. ${ }^{240}$ Mündliche Arbeitsverträge sind ebenso gültig, bieten jedoch im Streitfall zusätzliche Auslegungsschwierigkeiten. Das Obligationenrecht (OR) ist anwendbar, soweit der Arbeitsvertrag gewisse Punkte nicht regelt. Die nachfolgenden Ausführungen beschreiben die Bestimmungen des OR.

\section{Lohnzahlung im Krankheitsfall}

Kann ein Arbeitnehmer wegen Krankheit oder Unfall nicht arbeiten und hat die Arbeitgeberin keine Taggeldversicherung abgeschlossen, so muss die Arbeitgeberin im ersten Dienstjahr den Lohn während 3 Wochen bezahlen, danach während eines angemessenen längeren Zeitraums. ${ }^{241} \mathrm{Um}$ diesen angemessenen Zeitraum zu bestimmen, haben die Gerichte verschiedener Kantone eine Praxis entwickelt. Viele Kantone verwenden die sogenannte Berner-Skala: 1. Dienstjahr: 3 Wochen, 2. Dienstjahr: 1 Monat; 3. und 4. Dienstjahr: 2 Monate Lohnfortzahlung; usw.

Im Allgemeinen bezahlen die Taggeldversicherungen eine Entschädigung während 720 oder 730 Tagen, abzüglich der vorgesehenen Karenzfrist, über einen Zeitraum von gesamthaft 900 Tagen. Während der Karenzfrist muss der Arbeitgeber grundsätzlich den gesamten Lohn bezahlen.

\section{Kündigungsschutz bei Krankheit, Unfall, Militärdienst und Mutterschaft}

Nach Ablauf der Probezeit besteht ein Kündigungsschutz unter anderem im Krankheitsfall, bei Unfall, während des Militärdienstes, während der Schwangerschaft und in den 16 Wochen nach der Niederkunft (vgl. Kap. 10.5.). ${ }^{242}$ Bei ganzer oder teilweiser Arbeitsunfähigkeit infolge Krankheit oder Unfall gilt diese Sperrfrist für die Kündigung durch den Arbeitgeber während 30 Tagen im ersten Dienstjahr, 90 Tagen im zweiten bis fünften Dienstjahr und während 180 Tagen ab dem sechsten Dienstjahr. Dabei handelt es sich um Kalender- und nicht um Arbeitstage.

240 Die FMH stellt ihren Mitgliedern einen Musterarbeitsvertrag zur Verfügung. Dieser ist zwar kein obligatorischer Gesamtarbeitsvertrag. Die FMH empfiehlt aber dessen Verwendung. www.fmh.ch $\rightarrow$ Service $\rightarrow$ Medizinische Praxisassistentinnen $\rightarrow$ Musterarbeitsvertrag \& Lohnempfehlungen.

241 Art. 324a OR.

242 Art. 336c OR. 
Eine Kündigung während dieser Sperrfrist ist nichtig; der Arbeitgeber muss danach nochmals kündigen. Wenn die Kündigung hingegen vorher erfolgt ist, wird die Kündigungsfrist während der Sperrfrist sistiert und beginnt danach weiterzulaufen.

\section{Auflösung des Arbeitsverhältnisses}

Während der Probezeit können beide Parteien den Arbeitsvertrag zu jedem Zeitpunkt kündigen, wobei jedoch eine Kündigungsfrist von sieben Kalendertagen eingehalten werden muss. Die Probezeit darf höchstens drei Monate dauern.

Nach der Probezeit kann der Arbeitsvertrag je auf das Ende eines Monats gekündigt werden, wobei eine Kündigungsfrist von einem Monat im ersten Dienstjahr, zwei Monaten im 2. bis 9. Dienstjahr und 3 Monaten ab dem 10. Dienstjahr einzuhalten ist. Diese Fristen können durch den Arbeitsvertrag geändert werden. ${ }^{243}$

Beide Parteien können das Arbeitsverhältnis zu jedem Zeitpunkt «im gegenseitigen Einvernehmen» beenden - also ohne die Kündigungsfristen einzuhalten. In diesem Fall ist die Arbeitnehmerin nicht verpflichtet, bis ans Ende der Kündigungsfrist zu arbeiten, und der Arbeitgeber muss den Lohn für diesen Zeitraum nicht bezahlen.

\section{Ferien, freie Tage und Feiertage}

Der Arbeitnehmer hat pro Dienstjahr Anrecht auf mindestens vier Wochen Ferien. Bis zum erfüllten 20. Lebensjahr sind es mindestens fünf Wochen. Mindestens zwei Ferienwochen müssen zusammenhängen. ${ }^{244}$ Auch Teilzeitangestellte haben dasselbe Anrecht auf Ferien.

Dazu hat die Arbeitnehmerin die üblichen freien Tage (z.B. für einen Umzug oder eine Hochzeit) und die kantonalen Feiertage zugute. ${ }^{245}$ Das Gesetz sieht aber nicht vor, dass diese freien Tage und Feiertage bezahlt werden müssen, ausser für den Bundesfeiertag. In der Praxis spielt es jedoch keine Rolle, denn ein vereinbarter Monatslohn wird nicht gekürzt. 


\section{Berufliche Vorsorge}

Arbeitnehmer, die bei einem Arbeitgeber einen Jahreslohn von mehr als 20880 Franken beziehen, unterstehen der obligatorischen Versicherung. Die Risiken «Tod» und «Invalidität» müssen ab dem erfüllten 17. Lebensjahr und die Altersvorsorge ab dem erfüllten 24. Lebensjahr versichert werden. $\mathrm{Zu}$ versichern ist der koordinierte Lohn, das heisst der Teil des Jahreslohnes von 24360 bis und mit 83520 Franken. ${ }^{246}$ Pensionskassen können günstigere Versicherungsbedingungen und Vorsorgepläne anbieten als das gesetzliche Minimum.

\section{Arzt als Berufsbildner für Medizinische Praxisassistentinnen (MPA)}

Die FMH engagiert sich seit Jahrzehnten in der Ausbildung der MPA. Der ursprünglich privatrechtliche Beruf der Arztgehilfin DVSA wurde 1996 in einen eidgenössischen anerkannten und damit dem Bundesamt für Berufsbildung und Technologie unterstellten Beruf überführt. Das Ausbildungsreglement aus dem Jahr 1996 musste aufgrund des neuen Berufsbildungsgesetzes revidiert und in eine Bildungsverordnung und einen Bildungsplan umgegossen werden. Seit Inkrafttreten dieser neuen Grundlagen am 1. Januar 2010 kann mit der beruflichen Grundbildung neu direkt im Anschluss an die obligatorische Schulzeit begonnen werden. Daher ist für Berufsbildner insbesondere zu beachten, dass SchulabgängerInnen, die das 16. Lebensjahr noch nicht vollendet haben, nicht strahlenexponiert beschäftigt werden dürfen. ${ }^{247}$

Die Ausbildung findet wie bisher im Berufsschulunterricht, während den Überbetrieblichen Kursen sowie v.a. in der Arztpraxis statt. Der Bildungsplan gibt Auskunft, welche Lerninhalte während der 3-jährigen Ausbildung an welchen Ausbildungsorten zu vermitteln sind, wobei die Aufteilung der Schultage in den einzelnen Ausbildungsjahren kantonal unterschiedlich geregelt wird. Als Hilfsmittel sowohl für Berufsbildner als auch für Lernende liegt ein Praxisleitfaden vor, der sich an der täglichen Arbeit in der Arztpraxis orientiert und die Ausbildungsziele des Bildungsplans berücksichtigt. ${ }^{248}$

Wer eine MPA ausbilden möchte, benötigt eine Bildungsbewilligung des kantonalen Amtes für Berufsbildung. Das Amt ist zudem für die Genehmigung des Lehrvertrages, der in vielen Kantonen online über die Website des Amtes ausgefüllt werden kann, zuständig und ist Anlaufstelle in Problemsituationen. ${ }^{249}$

246 Diese Beträge werden in der Regel jährlich angepasst.

247 Strahlenschutzverordnung $\rightarrow$ www.admin.ch/ch/d/sr/c814_501.html

248 Informationen zur Ausbildung siehe www.mpaschweiz.ch

249 Die für eine Bildungsbewilligung erforderlichen Informationen und Formulare sowie Onlinelehrverträge sind auf den Websites der kantonalen Ämter für Berufsbildung aufgeschaltet (Adressen kantonaler Ämter $\rightarrow$ www.afb.berufsbildung.ch). 


\section{Praxisassistenz - ärztliche Weiterbildung in der Arztpraxis}

In einigen Weiterbildungsprogrammen ist die Anrechnung der Tätigkeit bei frei praktizierenden Ärzten an den angestrebten Facharzttitel vorgesehen. Voraussetzung ist jedoch, dass die Praxis vom SIWF im entsprechenden Fachgebiet als Weiterbildungsstätte anerkannt ist. ${ }^{250}$

Anrechenbar sind ununterbrochene Tätigkeiten von mindestens 1 Monat bis maximal 12 Monate, je nach Weiterbildungsprogramm. Wird der Assistent in dieser Zeit als Stellvertreter eingesetzt, kann dies nur in beschränktem Umfang und unter der Voraussetzung angerechnet werden, dass es das entsprechende Weiterbildungsprogramm erlaubt. Während der Weiterbildungsperiode ist vom Kandidaten das e-Logbuch zu führen. Am Ende der Weiterbildungsperiode muss der Lehrpraktiker das SIWF/FMH-Zeugnis aushändigen.

Das Programm «Weiterbildung in Hausarztpraxen (Praxisassistenz)», das die Stiftung zur Förderung der Weiterbildung in Hausarztmedizin (WHM) 2009 vom Kollegium für Hausarztmedizin übernommen hat, ermöglicht es den Weiterzubildenden, die spezifischen Fähigkeiten für eine spätere hausärztliche Tätigkeit direkt vor Ort in der Hausarztpraxis zu erwerben. Unter gewissen Voraussetzungen kann eine Mitfinanzierung bei der Stiftung WHM beantragt werden. Bedingung sind u.a. die Anerkennung als Weiterbildungsstätte durch das SIWF, ein Grundversorger-Facharzttitel und das Absolvieren eines Lehrpraktikerkurses des WHM. Geregelt wird das Anstellungsverhältnis mit einem vom WHM speziell ausgearbeiteten Vertrag. ${ }^{251}$

250 Die Informationen und das Antragsformular zur Anerkennung der Arztpraxis sind auf der Website des SIWF unter www.siwf.ch aufgeschaltet.

251 Alle Informationen zur Praxisassistenz WHM sind unter http://whm-fmf.ch/ aufgeschaltet. 


\title{
10.5. Jugendarbeitsschutz und Mutterschutz ${ }^{252}$
}

\author{
Bei Jugendlichen, Schwangeren und nach der Entbindung gelten besondere \\ Bestimmungen. Als Arbeitnehmerinnen und Arbeitnehmer stehen sie unter \\ einem erhöhten Schutz.
}

\section{Jugendschutz}

Das Arbeitsgesetz (ArG) bezeichnet Arbeitnehmer unter 18 Jahren als jugendliche Arbeitnehmer. ${ }^{253}$ Der Arbeitgeber muss ihre Gesundheit besonders schützen und ihre Arbeitszeit darf 9 Stunden pro Tag nicht überschreiten. ${ }^{254}$

Seit 1. Januar 2008 regelt die Jugendschutzverordnung die spezifischen Vorschriften für den «Schutz der Gesundheit und der Sicherheit der jugendlichen Arbeitnehmerinnen und Arbeitnehmer sowie ihrer physischen und psychischen Entwicklung». ${ }^{255}$

So ist Nacht- und Sonntagsarbeit im Grundsatz verboten, und es braucht eine Sonderbewilligung für Ausnahmen. Allerdings hat das Volkswirtschaftsdepartement für bestimmte Berufe systematische Ausnahmen während der Berufsbildung beschlossen. ${ }^{256}$

So dürfen im Gesundheitswesen beispielsweise unter 17-jährige Personen in Ausbildung zur MPA oder zur Pflegehilfe höchstens 2 Nächte pro Woche und höchstens 10 Nächte pro Jahr arbeiten. Sie dürfen auch einen Sonntag oder Feiertag pro Monat arbeiten, aber höchstens zwei Feiertage pro Jahr, die nicht auf einen Sonntag fallen. ${ }^{257}$

Personen, die bei ihrer Berufsausübung einer Strahlenbelastung ausgesetzt sind, müssen mindestens 16-jährig sein ${ }^{258}$ (vgl. auch Kap. 10.4.).

252 Vgl. zu diesen beiden Themen auch die Broschüren des Seco: «Mutterschaft - Schutz der Arbeitnehmerinnen" und "Jugendarbeitsschutz" unter www.seco.admin.ch $\rightarrow$ Dokumentation

$\rightarrow$ Publikationen und Formulare $\rightarrow$ Broschüren $\rightarrow$ Arbeit.

253 Art. 29 Abs. 1 ArG.

254 Art. 31 ArG.

255 Art. 5 Jugendarbeitsschutzverordnung, ArGV.

256 Verordnung des EVD vom 21. April 2011 über die Ausnahmen vom Verbot von Nacht- und Sonntagsarbeit während der beruflichen Grundbildung.

257 Art. 10 der Verordnung des EVD (siehe obenstehende Fussnote).

258 Art. 33 Abs. 3 der Strahlenschutzverordnung. 


\section{Gesundheitsschutz bei Mutterschaft ${ }^{259}$}

Der Arbeitgeber muss schwangere Frauen und stillende Mütter so beschäftigen und ihre Arbeitsbedingungen so gestalten, dass ihre Gesundheit und die Gesundheit des Kindes nicht beeinträchtigt werden. ${ }^{260}$

Schwangere und Stillende dürfen nur mit ihrem Einverständnis beschäftigt werden. In den ersten acht Wochen nach der Niederkunft gilt zudem ein absolutes Beschäftigungsverbot. Schwangere können überdies auf blosse Anzeige hin der Arbeit fernbleiben oder diese verlassen, und Stillenden muss der Arbeitgeber die erforderliche Zeit zum Stillen freigeben ${ }^{261}$; diese nichtkrankheitsbedingten Absenzen sind jedoch nicht bezahlt - es sei denn, die Schwangere hat ein Arztzeugnis oder es gibt eine besondere Vereinbarung für die Stillende.

Gefährliche und beschwerliche Arbeiten dürfen Schwangere und Stillende nur verrichten, wenn aufgrund einer Risikobeurteilung feststeht, dass dabei keine konkrete gesundheitliche Belastung für Mutter und Kind vorliegt, oder wenn eine solche durch geeignete Schutzmassnahmen ausgeschaltet werden kann. ${ }^{262}$

Für die Arztpraxis von Bedeutung sind insbesondere Arbeiten, bei denen die Schwangeren und Stillenden schädlichen Strahlen, Stoffen oder Mikroorganismen ausgesetzt sein könnten. Hinzu kommen das Bewegen von schweren Lasten sowie Bewegungen und Körperhaltungen, die zu vorzeitiger Ermüdung führen. Die FMH hat für die Arztpraxis ein Mutterschutzdossier ausarbeiten lassen und stellt die Vorlage für die Risikobeurteilung des Arbeitsplatzes, einen Musterbrief sowie ein ärztliches Zeugnis elektronisch zur Verfügung. ${ }^{263}$

Kann der Gefährdung nicht mit Schutzmassnahmen begegnet werden, so muss der Arbeitgeber die schwangere oder stillende Arbeitnehmerin an einen für sie ungefährlichen und gleichwertigen Arbeitsplatz versetzen. Kann er eine solche Ersatzarbeit nicht zuweisen, so hat die Arbeitnehmerin Anspruch auf $80 \%$ des Lohnes. Dies gilt auch, wenn auf ihr Verlangen anstelle von Nachtarbeit keine gleichwertige Tagesarbeit angeboten werden kann. ${ }^{264}$

Schwangere und Stillende dürfen nicht über die vereinbarte ordentliche Dauer der täglichen Arbeit hinaus beschäftigt werden und keinesfalls mehr als neun Stunden pro Tag. Schwangere dürfen ab der 8. Woche vor der Niederkunft zwischen 20 Uhr und 6 Uhr nicht beschäftigt werden.

259 Eine bildhafte Übersicht der Bestimmungen zum Mutterschutz findet sich unter www.fmh.ch $\rightarrow$ Service $\rightarrow$ MPA $\rightarrow$ Mutterschutz "Merkblatt zum Schutz der Arbeitnehmerin».

260 Art. 35 Abs. 1 und Abs. 3 ArG (Arbeitsgesetz, SR 822.11).

261 Art. 35a Abs. 1 ArG.

262 Art. 62 ArGV 1 (Verordnung 1 zum Arbeitsgesetz, SR 822.111).

263 www.fmh.ch $\rightarrow$ Service $\rightarrow$ MPA $\rightarrow$ Mutterschutz $\rightarrow$ "Risikobeurteilung des Arbeitsplatzes».

264 Art. 35 und Art. 35b ArG. 
Bei krankheitsbedingten Abwesenheiten während der Schwangerschaft richtet sich die Lohnfortzahlungspflicht primär nach dem Arbeitsvertrag, der allenfalls eine nichtobligatorische Krankentaggeldversicherung vorsieht. Liegt diesbezüglich keine vertragliche Regelung vor, gilt das Obligationenrecht.

\section{Kündigungsschutz bei Mutterschaft}

Nach Ablauf der Probezeit kann der Arbeitgeber einer Arbeitnehmerin während ihrer Schwangerschaft und in den 16 Wochen nach ihrer Niederkunft nicht kündigen. Eine Kündigung während dieser Sperrfrist ist nichtig, d.h., sie gilt als nicht erfolgt. ${ }^{265}$ Eine fristlose Kündigung aus wichtigen Gründen bleibt aber weiterhin möglich. Die Arbeitnehmerin ihrerseits kann das Arbeitsverhältnis jederzeit durch Kündigung auflösen. Zu beachten ist aber, dass eine Anstellung zum Zeitpunkt der Geburt Voraussetzung ist für den Bezug der Mutterschaftsentschädigung.

Arbeitgeber und Arbeitnehmerin können das Arbeitsverhältnis während der Sperrfrist durch eine gegenseitige Vereinbarung auflösen oder neu gestalten (z.B. Reduktion des Beschäftigungsgrades). In diesem Fall gelten keine Fristen, sondern der übereinstimmende Wille der Vertragsparteien. Aus Beweisgründen empfiehlt es sich, solche Vereinbarungen schriftlich zu verfassen.

\section{Mutterschaftsurlaub, Mutterschaftsentschädigung und Ferienanspruch}

Die Arbeitnehmerin hat nach der Niederkunft Anspruch auf einen Mutterschaftsurlaub von 14 bis 16 Wochen, je nachdem, ob sie unter das Arbeitsgesetz fällt. Während 14 Wochen erhält sie eine Mutterschaftsentschädigung in der Höhe von $80 \%$ des vorher erzielten Einkommens. Sie muss dafür im Zeitpunkt der Geburt angestellt sein. Der Anspruch erlischt, wenn sie vor Ablauf der 98 Tage wieder arbeitet; ${ }^{266}$ eine Unterbrechung des Mutterschaftsurlaubs ist nicht möglich. Der Anspruch wird in der Regel via Arbeitgeber bei der AHV-Ausgleichskasse geltend gemacht.

Die Ferien dürfen vom Arbeitgeber nicht gekürzt werden, wenn eine Arbeitnehmerin wegen Schwangerschaft bis zu zwei Monate an der Arbeitsleistung verhindert ist oder weil sie die Mutterschaftsentschädigung bezieht. ${ }^{267}$ 


\section{Anhang}

\section{Autorinnen und Autoren der zweiten Auflage}

lic. iur., dipl. biol. Matthis Bürgin, Basel

lic. iur. Jürg Gassmann, Rechtsanwalt, Zürich

lic. iur. Simone Grumbach, Rechtsanwältin, Bern

Christoph Hänggeli, Fürsprecher, Bern

Hanspeter Kuhn, Fürsprecher, Bern

lic. iur. Gabriela Lang, Rechtsanwältin, Bern

MLaw Barbara Linder, Bern

Lucia Rabia, Fürsprecherin, Bern

lic. iur. Valérie Rothhardt, Rechtsanwältin, Bern

Dr. iur. David Rüetschi, Zürich

lic. iur. Michelle Salathé, MAE, Basel

lic. iur. Simon Stettler, Fürsprecher, Bern

MLaw Anne-Sylvie Thiébaud, Rechtsanwältin, Bern

Dr. sc. hum. Judith C. Wagner, Bern

\section{Autorinnen und Autoren der ersten Auflage}

PD Dr. med. Georg Bosshard, MAE, Zürich

Michael Gerber, Fürsprecher, Bern

Prof. Dr. med. Bruno Gravier, Prilly

Christoph Hänggeli, Fürsprecher, Bern

lic. iur. Dania Ischi-Ceppi, Bern

lic. iur. Stephan Kaufmann, Basel

Hanspeter Kuhn, Fürsprecher, Bern

lic. theol. Peter Lack, Basel

Dr. med. et med. dent. Michael Mund, Zürich

Lucia Rabia, Fürsprecherin, Bern

lic. iur. Valérie Rothhardt, Rechtsanwältin, Bern

Dr. iur. David Rüetschi, Zürich

lic. iur. Michelle Salathé, MAE, Basel

lic. iur. Simon Stettler, Fürsprecher, Bern 
Wissenschaftlicher Beirat

Prof. Dr. iur. Christian Brückner, Basel

Prof. Dr. iur. Thomas Gächter, Zürich

Prof. Dr. iur. Thomas Geiser, St. Gallen

Michael Gerber, Fürsprecher, BAG, Bern

Prof. Dr. iur. Olivier Guillod, Neuchâtel

Prof. Dr. iur. Ulrich Meyer, Luzern

Prof. Dr. iur. utr. Brigitte Tag, Zürich

\section{Redaktion}

Dr. med. Hermann Amstad, Generalsekretär SAMW, Basel Hanspeter Kuhn, Fürsprecher, stv. Generalsekretär FMH, Bern lic. iur. Michelle Salathé, stv. Generalsekretärin SAMW, Basel 


\section{Stichwortverzeichnis}

\section{A}

Abtreibung (strafloser Schwangerschaftsabbruch) 77, 104

Akkreditierung $\mathbf{6 1}$

Anstellung am Spital oder in der Praxis $\mathbf{1 3 6}$

Arbeitgeber 109, 117, 143

Arbeitsunfähigkeit 109, 117

Armeeangehörige $\mathbf{7 0}$

Arzneimittel 48, 62

Arzneimittelliste mit Tarif (ALT) 62

Arzt-Patienten-Verhältnis 34

Arztdiplom 16, 53, 142

Arztgeheimnis 91, 98,104, 132

Arztpraxis 16, 35, 47, 128, 141, 146

Arztwahlfreiheit 38, 142

Arztzeugnis 108, 117

Ärzte und Industrie 19, 96

Asylsuchende 58, 91

AUF (siehe Arbeitsunfähigkeit)

Aufklärung des Patienten 21, 38 ,43, 46, 123, 128, 130

Auflösung des Arbeitsverhältnisses 144

Aufbewahrungsfrist $\mathbf{4 7}$

Aufsichtsbehörde 31

Aus-, Weiter- und Fortbildung 16

Auskunft an Elternteil 107, 127

Aussergewöhnlicher Todesfall 82, 105

B

Behandlung von Familienangehörigen 54

Behandlungsfehler 21, 38, 43, 46, 123, 128, 130

Behandlungsvertrag 34, 40, 116, 137

Behandlungszwang durch Strafrichter oder Versicherer $\mathbf{8 9}$

Beihilfe zum Suizid 81, 84

Beistandschaft 30, 43, 45, 77

Belegarzt 37, 138

Bericht 47, 108, 117, 128

Berufliche Vorsorge 145

Berufs- und Kassenzulassung 20

Berufsausübungsbewilligung 20, 139 
Berufsgeheimnis (siehe Arztgeheimnis)

Berufskrankheit 48, 68

Berufspflichten 20, 133

Betäubungsmittel (siehe Arzneimittel)

Betäubungsmittelmissbrauch, Melderecht bei 107

Betreibung 116

Bundesamt für Gesundheit (BAG) 17, 49, 52, 65, 95, 104

Bundesgesetz über den Versicherungsvertrag (VVG) 25

Bundesgesetz über die medizinisch unterstützte Fortpflanzung 76

Bundesgesetz über die Transplantation von Organen, Gewebe und Zellen 78

Bundesgesetz über genetische Untersuchungen am Menschen (GUMG) 74

Bundesverfassung 10

C

Case-Manager 111, 113

Critical Incident Reporting System (CIRS) 60

D

Datenschutz 90, 102, 128

Demenz 86

Dignität 67

Diplomanerkennung, gegenseitige 17

DNA-Profil 74

Dokumentation 36, 43, 47, 131

Doping 79

DSG Eidg. Datenschutzgesetz 102, 128

$\mathbf{E}$

Eidgenössische Arzneimittelkommission (EAK) 49

Eingliederungsmassnahmen 68, 119

Einsichtsrecht des Patienten in Krankengeschichte 103, 128

Eintrittsuntersuchung 109

Einwilligung 40, 43, 74, 93, 98, 104

Einsprache des Versicherten 66

Elektronische KG-Führung 47

Elektronischer Datenaustausch $\mathbf{1 0 3}$

Entscheidungsfindung 43

Epidemiengesetz 84, 104

Erwachsenenschutzbehörde 31

Erwachsenenschutzrecht 27, 80, 88 
Erwerbsunfähigkeit 118, 123, 138

Ethikkommission 94

Europäische Krankenversicherungskarte 54

F

Facharzt 16, 138

Fähigkeitsausweis 17, 53

Fahrtüchtigkeit, mangelnde 107

Familienangehörige, Behandlung von 54

Ferien 144, 149

FMH-Gutachterstelle 132

FMH-Tarifdienst 67

Forschung 48, 52, 58, 61, 93

Forschung am Menschen, Gesetz über (HFG) 11

Fortbildung 16, 18, 137

Fortpflanzungsmedizin $\mathbf{7 6}$

Franchise 55

Freie Arztwahl 38, 142

Freiheitsbeschränkung 86

Fremdgefährdung 86

Fristenlösung 77

Früherfassung 117

Fürsorgerische Unterbringung 30, 87

G

Generika 64

Genetische Untersuchungen 74, 120

Gesundheitsschaden 68, 117

Grundlagen des Rechts 9

Guidelines 13

GUMG (siehe genetische Untersuchungen)

Gutachten 108, 123

\section{H}

Haftpflicht 20, 39, 130, 133

Heilmittelgesetz 48, 71, 105

Heilversuch (siehe nichtetablierte Behandlung)

HFG (siehe Forschung am Menschen, Gesetz über)

HMG (siehe Heilmittelgesetz)

Hundebiss 104

Hungerstreik 90 
In-vitro-Fertilisation 76, 94

Industrie 96

Informationelles Selbstbestimmungsrecht 129

Informed consent 43, 83, 101

Invalidenversicherung 24, 68, 71, 117

Invalidität 118

\section{J}

Jugendschutz 147

K

Kantonale Gesetzgebung 12, 20, 35, 46, 50, 93, 100, 103, 129

Kantonale Paritätische Kommission (PVK) 67

Kassenpflichtigkeit 49, 54, 62

KLV (siehe Krankenpflege-Leistungsverordnung)

Kopierrecht des Patienten an Krankengeschichte 128

Körperschädigung 68

Korruption 138

Kostengutsprache 58, 63, 71

Kostenübernahmeprinzip $\mathbf{5 5}$

Krankengeschichte 36, 41, 46, 77, 90, 103, 128

Krankenpflege-Leistungsverordnung (KLV) 56

Krankenversicherungsverordnung 59

Krankenpflegeversicherung (siehe Krankenversicherung)

Krankenversicherung 11, 44, 54, 62, 75, 107

Krankenversicherungsgesetz (KVG) (siehe Krankenversicherung)

Krankheit 24, 55, 62, 68

Kündigungsschutz bei Krankheit, Unfall und Militärdienst 143

Kündigungsschutz bei Mutterschaft 143

KVG (siehe Krankenversicherung)

KVG-Zulassung 20, 57, 139

KVV (siehe Krankenversicherungsverordnung)

L

Labor 52, 56

Lebendorganspende 78

Lebensende 81, 85

Lebensversicherung 75, 119

Leichenschau 105

Leistungserbringer im KVG 56 
Leistungserbringer im KVG, MVG und IVG 69

Lohnzahlung im Krankheitsfall 143

\section{M}

Magistralrezeptur 49

Managed Care 58, 91

Medikamente (siehe Arzneimittel)

Medizinalberufegesetz 12, 16, 18, 61, 133, 139

Medizinialberufregister 16, 19

Medizinprodukte 48, 105

Medizinrecht 9

Medizinstudium 16

Meldepflicht 104, 118

Melderecht 104, 107, 117

MiGeL (Mittel- und Gegenstände-Liste im KVG) 62

Militärdienst 48, 70, 104, 117, 137, 143

Militärversicherung 68, 104

Minderjährige 34, 43, 78

Misshandlung 90

Mittel und Gegenstände 62

Mutterschaftsurlaub, Mutterschaftsentschädigung und Ferienentschädigung 149

Mutterschutz 147

\section{$\mathbf{N}$}

Naturalleistungsprinzip in UVG, MVG und IVG 69

Nichtetablierte Behandlung 52, 94

Notfalldienst 22

Nothilfepflicht 21

Notstandssituation 100, 102

0

Obduktion 106

Obligationenrecht (OR) 11, 35, 79, 136, 143

Off label use 51, 63

Öffentliches Spital 36

OKP (Obligatorische Krankenpflegeversicherung) (siehe Krankenversicherung)

Ombudsperson 96, 134

Organisationshaftung 136

Organtransplantation 78 
$\mathbf{P}$

PaKoDig (Paritätische Kommission Dignität) 67

Palliative Sedierung 83

Paritätische Interpretationskommission (PIK) 67

Paritätische Kommission Dignität (PaKoDig) 67

Paritätische Tarifkommission (PTK) 67

Paritätische Vertrauenskommission (PVK) 67, 69

Patientengeheimnis (siehe Arztgeheimnis)

Patientenpflichten 39

Patientensicherheit 46

Patientenverfügung 28, 45, 78, 82

Pensionskasse 24, 74, 119

Personenfreizügikeit (mit EU) 17, 54

Pflegeheim 38, 80

Pflichtleistung 55, 69

PIK (Paritätische Interpretationskommission) 67

Präimplantationsdiagnostik 76

Praxisassistenz 17, 146

Praxisbewilligung 139

Praxiseröffnung und Praxisübernahme 139

Praxisführung 142

Privatversicherungen (Zusatzversicherungen) 24, 75, 113

PTK (Paritätische Tarifkommission) 67

PVK (Paritätische Vertrauenskommission) 67, 69

$\mathbf{Q}$

Qualitätssicherung 17, 52, 59, 94

$\mathbf{R}$

RAD (Regionaler Ärztlicher Dienst der IV) 118

Reanimation 83

Rechenschaftspflicht 127

Rechnungsstellung 98, 113

Rechtsstreitigkeiten 66, 67, 123

Register 19, 23, 59, 94, 139

Rente (siehe Erwerbsunfähigkeit)

Rettungswesen 22

Rezeptformular 49

Richtlinien 12, 21, 78, 83, 85, 89, 96

Röntgen $\mathbf{5 2}$ 
Sans-Papiers 91

Schwangerschaftsabbruch 77, 104

Schwerpunkt 17, 67

Selbstbehalt 55

Selbstbehandlung des Arztes 54

Selbstbestimmungsrecht des Patienten 10, 27, 43, 86, 129

Selbstdispensation 49

Selbstgefährdung 86

Selbstverschulden 55, 69

Sexueller Übergriff 134

Sozialversicherungen 24, 55, 59, 67, 111, 119, 136

Spenderausweis 78

Spezialgesetze 11

Spezialitätenliste 62

Sportmedizin $\mathbf{7 9}$

Stammzellenforschung $\mathbf{9 4}$

Standeskommission 21, 134

Standesordnung 12, 20, 23, 35, 46, 79, 99, 108, 120, 133, 137

Stellvertretung 142

Sterbehilfe 11, 81, 83

Sterilisation 11, 77

Steuern 137

Strafbare Handlungen an Unmündigen 107

Strafverfahren 133

Strafvollzug 89

Strahlenschutz 52

Strassenverkehrsgesetz 107

Suizid, Beihilfe zu $\mathbf{8 4}$

Suva 70, 117

Swissmedic 49, 105

$\mathbf{T}$

Taggeld 24, 69, 75, 89, 109, 117, 138, 143

Taggeldversicherer (siehe Taggeld)

Tarifrecht 65

TARMED 18, 22, 60, 65, 69, 110

Totenschein 105

Touristen 54

Transplantationsgesetz 11, 78

Trust Center 113 
U

UAW (Unerwünschte Arzneimittelwirkung), Meldepflicht bei 105

Übertragbare Krankheiten 10, 104

UKS (Unfallähnliche Körperschädigung) 68

Unfall 24, 68, 117, 123, 125, 143

Unfallversicherung (siehe Unfall)

Unlicensed use 51, 63

Unzufriedener Patient 134

Urteilsfähigkeit 27, 34, 43, 44, 77, 85

Urteilsunfähigkeit (siehe Urteilsfähigkeit)

V

Verjährungsfrist 39

Vertragsfreiheit 24, 119

Verordnung über die klinischen Versuche (VKlin) 105

Vergünstigungen, Weitergabe von 65

Versichertenkarte 54

Versicherungsobligatorium 54

Versicherungsvorbehalt 25, 37, 119

Vertrauensarzt 55, 111

Vertretung bei medizinischen Massnahmen 11, 28, 44

Vorbescheidsverfahren 72

Vorsorgeauftrag 27

\section{W}

Wachkoma 83

Weiterbildung 16, 50, 53, 67, 74, 142, 146

Werbung 20, 23, 142

Wissenschaftliche Integrität 95

WZW-Kriterien 71

\section{Z}

Zertifizierung 59, 61

Zeugnis 100, 108, 117, 119, 121

ZSR-Nummer 57, 139

Zulassungsstopp 49, 112

Zusatzversicherung (siehe Privatversicherungen)

Zwangsbehandlung (siehe Zwangsmassnahmen)

Zwangsmassnahmen 86 


SAMW Schweizerische

Akademie der Medizinischen

Wissenschaften 